\title{
Inclusive production through AdS/CFT
}

\section{Richard Nally, ${ }^{a, b}$ Timothy Raben ${ }^{a, c}$ and Chung-I Tan ${ }^{a}$}

${ }^{a}$ Department of Physics, Brown University, 182 Hope St., Providence, RI, U.S.A.

${ }^{b}$ Department of Physics, Stanford University, 382 Via Pueblo Mall, Stanford, CA, U.S.A.

${ }^{c}$ Department of Physics and Astronomy, University of Kansas, 1251 Wescoe Hall Dr., Lawrence, KS, U.S.A.

E-mail: rnally@stanford.edu, timothy.raben@ku.edu, tan@het.brown.edu

ABSTRACT: It has been shown that AdS/CFT calculations can reproduce certain exclusive $2 \rightarrow 2$ cross sections in QCD at high energy, both for near-forward and for fixed-angle scattering. In this paper, we extend prior treatments by using AdS/CFT to calculate the inclusive single-particle production cross section in QCD at high center-of-mass energy. We find that conformal invariance in the UV restricts the cross section to have a characteristic power-law falloff in the transverse momentum of the produced particle, with the exponent given by twice the conformal dimension of the produced particle, independent of incoming particle types. We conclude by comparing our findings to recent LHC experimental data from ATLAS and ALICE, and find good agreement.

KEYWORDS: Strings and branes phenomenology, QCD Phenomenology

ARXIV EPRINT: 1702.05502 


\section{Contents}

1 Introduction $\quad 2$

2 Inclusive cross sections and discontinuities $\quad 6$

$\begin{array}{lll}2.1 & \text { 2-point functions } & 6\end{array}$

$\begin{array}{lll}2.2 & \text { Inclusive distributions in CFT } & 7\end{array}$

3 String-gauge duality $\quad 9$

3.1 AdS scattering 9

3.2 Inclusive cross sections as AdS discontinuities 13

4 Inclusive single-particle production in the central region $\quad 14$

$\begin{array}{ll}\text { 4.1 Inclusive central production and the 3-to-3 amplitude } & 15\end{array}$

$\begin{array}{lll}4.2 & \text { Central production at large } p_{\perp} \text { and conformal invariance } & 18\end{array}$

5 Evidence for conformality $\quad 19$

5.1 Proton-lead collisions and pseudorapidity dependence 21

5.2 Proton-proton collisions and center of mass energy dependence 22

$\begin{array}{lll}5.3 & \text { Interpretation } & 23\end{array}$

6 Summary and discussion $\quad 26$

A Inclusive cross sections and applications $\quad \mathbf{2 7}$

$\begin{array}{ll}\text { A.1 Single particle inclusive } & 28\end{array}$

A.2 DIS, OPE and anomalous dimensions 29

$\begin{array}{ll}\text { B AdS/CFT scattering and the BPST program } & 30\end{array}$

C Conformal partial-wave and Regge theory $\quad 34$

C.1 OPE in Minkowski setting 35

C.2 Conformal Regge theory and eikonal 36

$\begin{array}{ll}\text { D Flat-space string amplitudes } & 36\end{array}$

$\begin{array}{llr}\text { E } & \text { Fit validation and parameter stability } & 39\end{array}$

E.1 Power-law behavior $\quad 39$

$\begin{array}{lll}\text { E.2 Small } p_{T} & 48\end{array}$ 


\section{Introduction}

It has been suspected for many years that large- $N_{c}$ QCD admits an alternate description as a string theory. ${ }^{1}$ Early developments were inspired by the realization that string scattering amplitudes obey Regge behavior and crossing symmetry. This conjecture was greatly spurred on with the observation that, in the limit of large $N_{c}$ with $\lambda=g_{Y M}^{2} N_{c}$ fixed, the QCD perturbation series can be made to resemble the genus expansion of worldsheet string theory [2]. With the advent of AdS/CFT correspondence [3-8], or equivalently gauge-string duality, the theoretical landscape has taken a dramatic step forward and a string realization of QCD has again become a serious goal for current studies.

In this paper, we explore the consequences of conformal symmetry in high energy scattering experiments. In particular, we will use the AdS/CFT correspondence to examine inclusive production. Although strictly speaking QCD itself is not a CFT, it is closely related to $\mathcal{N}=4$ super Yang-Mills, which is conformal, and the two theories are similar enough that a great deal can be learned from the conformal limit [9-12]. The effects of conformal symmetry on QCD have previously been studied in inclusive scattering in both the fixed-angle $[13,14]$ and in the near-forward limits [15-25]. Here, we will focus on central production at the LHC.

Inclusive processes unavoidably involve near-forward particle production. The relevant physics is intrinsically non-perturbative, and cannot be reduced simply to purely partonic scattering. With AdS/CFT, one is able to address both perturbative and non-perturbative aspects of inclusive production at high energy in a unified setting. Indeed, holographic techniques based on a $t$-channel OPE [26-33] have been used as a complement to more traditional weak coupling methods [34-39] to study HERA data for the deep inelastic scattering (DIS) cross section at large $s$ and small $x=Q^{2} / s$.

Early interest in inclusive production can be traced back to the work of Feynman [40], Yang [41], Wilson [42], and others, focusing particularly on the scaling properties of particle distributions. Studies of inclusive production in a CFT context began with works of Strassler [43], Hofman and Maldecena [44] and Belitsky et al. [45-47]. Instead of focusing on the final state particle distribution, which is ill-defined in the strict CFT limit, the emphasis has been on infrared safety [48, 49], e.g., on energy flows, leading to vacuum expectations

$$
\sigma_{w}(p)=\int d^{4} x e^{-i p x}\left\langle 0\left|\mathcal{O}^{\dagger}(x) \mathcal{D}[w] \mathcal{O}(0)\right| 0\right\rangle .
$$

Here $\mathcal{O}$ serves as the source for the initial state $|\mathcal{O}(p)\rangle$, which carries 4 -momentum $p_{\mu}$, and $\mathcal{D}[w]$ is a product of a set of local operators, measuring flows of conserved quantities, such as energy-momentum; such an object is generically referred to as an inclusive "event shape" distribution.

The operator product $\mathcal{D}[w]$ in the above expression is not time-ordered, and thus the appropriate Lorentzian correlation functions are Wightman functions. Momentum space Wightman functions lead to amplitude discontinuities, so it is necessary to deal

\footnotetext{
${ }^{1}$ See [1] for a detailed historical discussion.
} 
with Landau-Cutkosky singularities. ${ }^{2}$ The treatments in [43-47] have mainly focused on processes where the source involves a single local operator, such as $e^{+} e^{-} \rightarrow \gamma^{*} \rightarrow X$, where $X$ represents all allowed final states, which are implicitly summed over.

Our discussion in this paper will deal primarily with scattering processes where the initial source is non-local, and will be carried out in a momentum representation. The simplest inclusive scattering process is

$$
a+b \rightarrow X
$$

where again $\mathrm{X}$ implies a sum over all possible final states. After summing over contributions from all possible final states, the completeness relation $\sum_{X}|X\rangle\langle X|=I$ leads to the usual optical theorem, which states that the total cross section $\sigma_{\text {total }}^{a b}(s)$ of such a process is given by the imaginary part of the elastic amplitude in the forward limit, ${ }^{3}$

$$
\sigma_{\text {total }}^{a b}(s) \simeq \frac{1}{s} \operatorname{Im} T(s, t=0) .
$$

The next simplest inclusive process is single particle production,

$$
a+b \rightarrow c+X
$$

where again $\mathrm{X}$ implies a sum over all possible final states, leading to a differential production cross section, $d \sigma_{a b \rightarrow c+X} / d^{3} \mathbf{p}_{c}$. Kinematically, single particle production can be treated as a 2-to-2 process, with $\mathrm{X}$ having a variable mass $M_{X}^{2}=\left(p_{a}+p_{b}-p_{c}\right)^{2}$ often referred to as the "missing mass"; for simplicity, we will simply call this $M^{2}$. The invariant differential cross section $d \sigma_{a b \rightarrow c+X} /\left(d^{3} \mathbf{p}_{c} / E_{c}\right)$ therefore depends on three Lorentz invariants instead of the usual two for exclusive scattering. The usual Mandelstam variables $s, t$, and $u$ can be used, but it is frequently more convenient to work with $s, t$, and $M^{2}$; it is easy to see that $s+t+u=m_{a}^{2}+m_{b}^{2}+m_{c}^{2}+M^{2}$, so these two sets of variables encode the same information.

In a momentum space treatment, inclusive cross sections can always be identified as discontinuities of appropriate forward amplitudes through the use of generalized optical theorems. The differential cross section $d \sigma_{a b \rightarrow c+X} /\left(d^{3} \mathbf{p}_{c} / E_{c}\right)$ of the process $a b \rightarrow c+X$ can be identified as the discontinuity in $M^{2}$ of the amplitude for the six-point process $a b c^{\prime} \rightarrow a^{\prime} b^{\prime} c$; symbolically, we have

$$
\frac{d \sigma_{a b \rightarrow c+X}}{d^{3} \mathbf{p}_{c} / E_{c}} \simeq \frac{1}{2 i s} \operatorname{Disc}_{M^{2}} T_{a b c^{\prime} \rightarrow a^{\prime} b^{\prime} c}
$$

The main goal of this paper is to explore the consequences of conformality on inclusive central production in proton-proton and proton-lead scattering. We examine the use of the $t$-channel OPE $[15,51]$ for high energy scattering, elucidate subtleties involved in using generalized optical theorems, and pay special attention to non-perturbative issues. In

\footnotetext{
${ }^{2}$ The Landau-Cutkosky singularities for Lorentzian correlation functions in CFTs with a gravity dual has recently been addressed in [50].

${ }^{3}$ Here we use canonically defined Mandelstam invariants. The elastic scattering amplitude $T(s, t)$ is parameterized by the usual center of mass energy squared $s$ and the momentum transfer squared $t$.
} 
particular, we show that aspects of conformal invariance can be explored in a "gluon-rich" environment ${ }^{4}$ by treating central inclusive particle production of the form

$$
a+b \rightarrow X_{1}+c+X_{2},
$$

where $X_{1}$ and $X_{2}$ represent left- and right-moving "lumps" in the CM frame.

Our discussion can be divided into several parts. We first focus on the more formal question of how to treat CFT inclusive shape distributions as weighted discontinuities of multiparticle momentum space amplitudes $T_{a b 1^{\prime} 2^{\prime} \ldots \rightarrow a^{\prime} b^{\prime} 12 \ldots}$, generalizing on earlier treatments. This treatment is carried out necessarily in a Minkowski setting, with the discontinuity in the generalized missing mass, $M^{2}=\left(p_{a}+p_{b}-\sum p_{i}\right)^{2}$ taken in the forward limit. This procedure applies to both events initially sourced by a single local operator, as in eq. (1.1), and to scattering processes at high energy, as in eqs. (1.3) and (1.5).

By multiparticle amplitudes here we simply refer to the usual Euclidean CFT correlations functions, $\left\langle\varphi\left(x_{1}\right) \varphi\left(x_{2}\right) \cdots\right\rangle$, continued to Lorentzian signature; these lead to vacuum expectation values for time-ordered (or $\mathbf{T}$ product) conformal primaries, $\left\langle 0\left|\mathbf{T}\left\{\varphi\left(x_{1}\right) \varphi\left(x_{2}\right) \cdots\right\}\right| 0\right\rangle$. We assume a standard Hilbert space structure (e.g. a state space spanned by states associated with conformal primaries) which allow us to use completeness relations. Although our emphasis is on purely conformal characteristics, we are mainly concerned with theories that allow an IR confinement deformation so one can interpret the results in terms of canonically defined scattering amplitudes.

We will next discuss inclusive production for scattering processes and explore in particular the consequences of AdS/CFT and conformal invariance at finite 't Hooft coupling, $\lambda$ large but finite. Here we review the bare necessities on how to move beyond the supergravity limit by including string corrections, and so we are effectively dealing with string amplitudes on an AdS background. Historically, the greatest obstacle to a stringy description of QCD phenomenology has been the requirement of hard partonic behavior at short distances. AdS/CFT provides a framework to resolve these phenomenological difficulties. Polchinski and Strassler [13] identified an approximation regime in which the warped geometry of the dual AdS theory provided a power-law falloff for wide-angle scattering in QCD. This argument has been extended to near-forward QCD scattering in AdS/CFT. We follow a similar approach as first described in $[15,52]$. In [15] it was shown that Pomeron exchange, i.e. the leading Regge singularity with the quantum numbers of the vacuum, can be described by a Reggeized graviton ${ }^{5}$ propagating in $\mathrm{AdS}_{5}$. The unifying principle for both exclusive power-behavior at the fixed-angle limit and the Pomeron dominance for the near-forward scattering is conformal invariance.

We next apply our analysis to single-particle inclusive production in the central region. Here $X$ can be separated into left- and right-moving groups, $X_{1}$ and $X_{2}$ respectively. The event shape distribution is controlled by a matrix element, $\left\langle\mathcal{V}_{P} V_{c \bar{c}} \mathcal{V}_{P}\right\rangle$, involving two Pomeron vertex operators [15]. Just as the case of exclusive fixed-angle scattering, flat

\footnotetext{
${ }^{4}$ In an AdS/CFT treatment, QCD is dominated by gluon dynamics with quark loop contributions suppressed. These contributions can become important in the "fragmentation regions" however.

${ }^{5}$ In what follows, this will also be referred to as the BPST pomeron [15]. This stands in contrast with the BFKL Pomeron [53-55], which is based on perturbative QCD.
} 
space string scatting amplitudes [56-60] predict an exponential cutoff in the transverse momentum $\sim e^{-4 \alpha^{\prime} p_{\perp}^{2}}$. However, we argue that a generalization of the Polchinski-Strassler regime $[13,14]$ utilizes the warped AdS geometry to render the effect of confinement deformation unimportant at high $p_{T}$. Using this we arrive at our central result for CFT behavior at the LHC involving a partonic power-law falloff of the form

$$
\frac{d \sigma_{a b \rightarrow c+X}}{d^{3} \mathbf{p}_{c} / E_{c}} \sim \operatorname{Disc}_{M^{2}}\left\langle\mathcal{V}_{P} V_{c \bar{c}} \mathcal{V}_{P}\right\rangle \sim p_{\perp}^{-\delta}
$$

The exponent $\delta$ is fixed by holography and conformal invariance, given by $\delta=2 \tau$, with $\tau=\Delta-J$, where $\Delta$ is the conformal dimension, and $J$ the spin of the produced hadron. ${ }^{6}$ In the large $N_{c}$ limit of the AdS/CFT, the theory is dominated by gluonic interactions; the production of fermion pairs is suppressed by $1 / N_{c}$. The simplest bound state is then a glueball state $\operatorname{Tr}\left(F^{2}\right)$ [63-65] which can be used to describe meson production via AdS/CFT [29, 6669]. ${ }^{7}$ In QCD, scattering processes dominated by Pomeron exchange are described via the BFKL Pomeron (reviewed in [71]). Since the BFKL Pomeron the exchange of a Reggeized gluon ladder, the bound states lying on the trajectory are thought to be glueballs. ${ }^{8}$ For production via scalar glueballs, we thus have $\delta=2 \Delta=8$. This is analogous to the dimensional counting rule [73-75], but from a non-perturbative perspective. Finally, we test this prediction by comparing to recent ATLAC and ALICE data from the LHC.

This paper is organized as follows: in section 2, we focus on the treament of inclusive distributions as discontinuities. Section 2.1 involves reviewing the simple, but illustrative case of 2 point functions. Although these results can be found in the literature, we re-derive them in a consistent notation. Using this notation we then reinterpret known results about 4- and 6-point functions and present new analysis about generalized n-point functions in section 2.2. Elucidating examples are left to appendix A.2. In section 3, by invoking AdS/CFT, we express inclusive cross sections in terms of the discontinuities of Witten diagrams. In section 3.1 we detail 2-point functions in AdS and derive analogous results to those in section 2. Following this we are able to posit our prediction for high energy inclusive scattering in section 3.2. We turn next, in section 4, to inclusive distribution in the central production. Finally, in section 5, we test this finding by comparing with the recent LHC data; in section 5.3 we discuss possible explanations for the results of the experimental fits. We conclude with a brief discussion of our essential results in section 6 .

Throughout the paper, the details of results from earlier literature are omitted from the body of the text, and are instead provided in appendices A-D. In particular, these appendices cover the treatment of inclusive cross sections as discontinuities in QCD itself, the holographic pomeron, aspects of conformal field theory, and flat space string amplitudes, respectively. However, because much of the work here connects disparate background ma-

\footnotetext{
${ }^{6}$ Amplitudes displaying a similar power-law like behavior can be described using a complimentary holographic approach where one simply considers the string zero-mode contribution. Further details can be found in [61, 62] and references therein. We focus here on the BPST approach as we believe it is more analogous to the perturbative weak coupling approach where in both cases Regge poles can be interpreted as eigenvalues of an effective Hamiltonian approach.

${ }^{7}$ For a brief introduction to mesons in AdS/CFT see [70].

${ }^{8}$ For a recent review of the Pomeron/Glueball connection see [72].
} 
terial we provide a bare minimum of review and examples in the main text for the paper to be relatively self contained.

\section{Inclusive cross sections and discontinuities}

In field theory, inclusive cross sections involve Minkowski space Wightman functions. In this section, we clarify how these Wightman functions, in a momentum representation, can be identified as "forward discontinuities" of $n$-to- $n$ amplitudes, e.g., $n=3$ for the process $a+b \rightarrow c+X$. We begin by reviewing the more familiar case of 2-point functions before generalizing to higher point correlators. We conclude by demonstrating these ideas in the context of deep inelastic scattering (DIS), where the cross section can be explicitly related to a discontinuity; we also relate the moments of the DIS distribution to a $t$-channel OPE.

\subsection{2 -point functions}

The relationship between a conventional time-ordered Green's function, $\left\langle 0\left|\mathbf{T}\left\{\varphi_{1} \varphi_{2} \cdots\right\}\right| 0\right\rangle$, and a Wightman function, which is not time-ordered, can best be understood in a momentum representation. Let us illustrate this by first comparing the Feynman propagator, $\langle 0|\mathbf{T}\{\varphi(x) \varphi(0)\}| 0\rangle$, for a free scalar, with the corresponding Wightman function, $\langle 0|\varphi(x) \varphi(0)| 0\rangle$. In a momentum representation, $, 9,10$

$$
\begin{aligned}
G_{F}\left(p^{2}\right) & =i \int d^{4} x e^{i p \cdot x}\langle 0|T(\varphi(x) \varphi(0))| 0\rangle=-\frac{1}{p^{2}-m^{2}+i \varepsilon}, \\
G_{W}\left(p^{2}\right) & =\int d^{4} x e^{i p \cdot x}\langle 0|\varphi(x) \varphi(0)| 0\rangle=2 \pi \delta\left(p^{2}-m^{2}\right) \theta\left(p^{0}\right),
\end{aligned}
$$

$G_{F}\left(p^{2}\right)$ is an analytic function in the invariant $p^{2}$, with poles at $p^{0}= \pm \sqrt{m^{2}+\vec{p}^{2}}$. However, $G_{W}\left(p^{2}\right)$ defines a distribution, corresponding to the discontinuity of $G_{F}\left(p^{2}\right)$ for $p^{0}>0$.

Let us turn next to CFT, using conventional CFT normalization and again in a Minkowski setting. Consider a generic scalar conformal primary $\varphi$ of dimension $\Delta$. The Fourier transform for its Feynman propagator and the corresponding Wightman function are

$$
\begin{aligned}
G_{F}\left(p^{2}\right) & =i \int d^{4} x \frac{e^{i p x}}{\left.\left[\vec{x}^{2}-t^{2}+i \varepsilon\right)^{2}\right]^{\Delta}}=-d(\Delta)\left(-p^{2}\right)^{\Delta-2}, \\
G_{W}\left(p^{2}\right) & =\int d^{4} x \frac{e^{i p x}}{\left[\vec{x}^{2}-(t-i \varepsilon)^{2}\right]^{\Delta}}=c(\Delta) \theta\left(p^{2}\right) \theta\left(p^{0}\right)\left(p^{2}\right)^{\Delta-2},
\end{aligned}
$$

where $c(\Delta)=2 d(\Delta) \sin \pi \Delta=(2 \pi)^{3} 2^{2(1-\Delta)} / \Gamma(\Delta) \Gamma(\Delta-1) . G_{F}\left(p^{2}\right)$ is a real-analytic function $^{11}$ in $p^{2}$, with a branch cut across $0<p^{2}<\infty$. The corresponding Wightman propagator, $G_{W}\left(p^{2}\right)$, is a distribution. Although there is no mass-gap, the relation between

\footnotetext{
${ }^{9}$ In this paper, we adopt the $(+,-,-,-)$ metric, so that $p^{2}=p^{0^{2}}-\vec{p}^{2}$ and $p \cdot x=p^{0} t-\vec{p} \cdot \vec{x}$.

${ }^{10}$ With interactions, in addition to the pole, $G_{F}\left(p^{2}\right)$ acquires a branch cut for $p^{2}>4 m^{2}$, and can be represented in a spectral representation, again with $G_{W}\left(p^{2}\right)$ as its discontinuity, for $p^{0}>0$.

${ }^{11}$ Positivity requires that $1 \leq \Delta$. For $\Delta$ approaching a positive integer $n$, coefficient $d$ diverges while $c$ remains finite, indicating the emergence of $\left(-p^{2}\right)^{n-2} \log \left(-p^{2}\right)$. We shall stay with a generic $\Delta$, away from positive integers.
} 
time-ordered amplitudes and Wightman functions remains. $G_{W}\left(p^{2}\right)$ is a continuum over $0<p^{2}<\infty$, corresponding to the discontinuity of $G_{F}\left(p^{2}\right)$ across its cut.

\subsection{Inclusive distributions in CFT}

It is useful to distinguish between two types of inclusive processes. The first type corresponds to events with a single initial local source, e.g. $\gamma^{*} \rightarrow c_{1}+c_{2}+\cdots+X$, which has been discussed before. The second type invovles a non-local source, as in scattering, e.g., $a+b \rightarrow c_{1}+c_{2}+\cdots+X$, which we expand on here. In the language of CFT, these inclusive cross sections can be interpreted as flow rates for conserved quantities, such as the energy density flowing into a solid angle $d^{2} \Omega$ about a direction $\hat{n}$ [44-47]. General inclusive flows for conserved quantities can always be expressed as weighted discontinuities [44-47].

Let us consider scattering processes first. As stated in section 1, the simplest inclusive process corresponds to eq. (1.2), with the total cross section given by the imaginary part of forward amplitude, as in eq. (1.3). Consider next the inclusive production of a scalar particle, $a+b \rightarrow c+X$, as in eq. (1.4). The invariant differential cross section can be expressed as [76]

$$
\begin{aligned}
\frac{d \sigma_{a b \rightarrow c+X}}{d^{3} \mathbf{p}_{c} / E_{c}} & \propto \sum_{X}(2 \pi)^{4} \delta^{(4)}\left(p_{a}+p_{b}-p_{c}-p_{X}\right)\left|\left\langle p_{c}, X \mid p_{a}, p_{b}\right\rangle\right|^{2} \\
& \propto \sum_{X} \int d^{4} x e^{-i p_{c} \cdot x}\left\langle p_{a}, p_{b}\left|\varphi_{c}(x)\right| X\right\rangle\left\langle X\left|\varphi_{c}(0)\right| p_{a}, p_{b}\right\rangle .
\end{aligned}
$$

Making use of the completeness relation $\sum_{X}|X\rangle\langle X|=I$, the cross section can also be expressed as a matrix element,

$$
\frac{d \sigma_{a b \rightarrow c+X}}{d^{3} \mathbf{p}_{c} / E_{c}} \propto\left\langle p_{a}, p_{b}\left|\widetilde{\mathcal{O}}_{c}\left(p_{c}\right)\right| p_{a}, p_{b}\right\rangle
$$

where $\widetilde{\mathcal{O}}_{c}=\int d^{4} x e^{-i p_{c} \cdot x} \varphi_{c}(x) \varphi_{c}(0)$ is the Fourier transform of product of two local operators. Here, $p_{c}$ is the four-momentum for the produced scalar, with $p_{c}^{0}>0$. Since the product $\varphi_{c}(x) \varphi_{c}(0)$ is not time-ordered, one is again dealing with a Wightman function.

The corresponding 3-to-3 process is $a+b+c^{\prime} \rightarrow a^{\prime}+b^{\prime}+c$, where the amplitude $T_{a b c^{\prime} \rightarrow a^{\prime} b^{\prime} c}$ is given by a $\mathbf{T}$-product between asymptotic states, ${ }^{12}\left\langle p_{a^{\prime}}, p_{b^{\prime}}\left|\mathbf{T}\left\{\varphi_{c}(x) \varphi_{c}(y)\right\}\right| p_{a}, p_{b}\right\rangle$, in momentum space. One can move from a $\mathbf{T}$-product to a Wightman function as done earlier for the free propagator. Because it is a matrix element between asymptotic states, one replaces the 4 -vector $p$ in $(2.2)$ by $\left(p_{a}+p_{b}-p_{c}\right)$, with $p_{a}^{0}+p_{b}^{0}-p_{c}^{0}>0$ and $p_{c}^{0}>0$. Therefore, $\left\langle p_{a}, p_{b}\left|\widetilde{\mathcal{O}}_{c}\left(p_{c}\right)\right| p_{a}, p_{b}\right\rangle$ is the discontinuity of $T_{a b c^{\prime} \rightarrow a^{\prime} b^{\prime} c}$, in the invariant $M^{2}=\left(p_{a}+p_{b}-p_{c}\right)^{2}$,

$$
\frac{d \sigma_{a b \rightarrow c+X}}{d^{3} \mathbf{p}_{c} / E_{c}} \propto \frac{1}{2 i s} \operatorname{Disc}_{M^{2}>0} T\left(p_{a^{\prime}}, p_{b^{\prime}}, p_{c} ; p_{a}, p_{b}, p_{c^{\prime}}\right) .
$$

\footnotetext{
${ }^{12}$ Energy components for all external 4 -vectors are positive. For simplicity, the overall delta-function due to translational invariance will be surpressed in what follows. Strictly speaking, we need to work with amputated on-shell amplitudes, where $\varphi_{c}$ should be replaced by a source function, $j_{c}(x)=\left(\square-m_{c}^{2}\right) \varphi_{c}(x)$. We will skip this step to avoid notational overload.
} 
This is the process that is examined holographically in section 4 and more details can be found in appendix A.

Next we turn to inclusive processes involving a single local source, $\mathcal{O}$, for example $e^{+} e^{-} \rightarrow \gamma^{*}(p) \rightarrow c_{1}+c_{2}+\cdots+X$. The decay process can be interpreted as a CFT process as motivated by the work of Hofman and Maldacena [44]. In what follows it will be useful to recast eq. (1.1) in the form of a normalized distribution in a momentum representation as

$$
\left\langle\widetilde{O}_{w}\right\rangle=\frac{\sigma_{w}(p)}{\sigma_{\mathcal{O}}(p)}=\frac{\int d^{4} x e^{i p x}\left\langle 0\left|\mathcal{O}^{\dagger}(x) \widetilde{O}_{w} \mathcal{O}(0)\right| 0\right\rangle}{\int d^{4} x e^{i p x}\left\langle 0\left|\mathcal{O}^{\dagger}(x) \mathcal{O}(0)\right| 0\right\rangle}=\frac{\left\langle\mathcal{O}(p)\left|\widetilde{O}_{w}\right| \mathcal{O}(p)\right\rangle}{\langle\mathcal{O}(p) \mid \mathcal{O}(p)\rangle},
$$

where $\widetilde{O}_{w}$ is chosen to ensure infrared safety. In general, $\widetilde{O}_{w}$ is a non-time-ordered product of a set of local operators, as in eq. (2.6); as discussed above, this necessitates the use of Wightman functions [45-47].

We can now apply to this expression the same analysis used to argue for eq. (2.5). The matrix element $\left\langle\mathcal{O}(p)\left|\widetilde{O}_{w}\right| \mathcal{O}(p)\right\rangle$ admits a form similar to eq. (2.4), but with the momentum $p$ replaced with $p-p_{c}$, where $p_{c}$ is the momentum associated with the flow so that $p^{0}>$ $p_{c}^{0}>0$. Then we can relate $\left\langle\mathcal{O}(p)\left|\widetilde{O}_{w}\right| \mathcal{O}(p)\right\rangle$ to a discontinuity exactly as was done for eq. (2.5) earlier.

Generically, we can write the cross section for such a process as

$$
\sigma_{w}(p)=\sum_{X}(2 \pi)^{4} \delta^{(4)}\left(p-p_{X}\right) w(X)\left|\left\langle X \mid \gamma^{*}(p)\right\rangle\right|^{2},
$$

where the sum is taken over all possible $X$ and involves an integration over the phase space for each state $X$, weighted by $w(X)$. For example, the simplest inclusive single-particle production process, $e^{+} e^{-} \rightarrow \gamma^{*} \rightarrow c+X$, involves the measurement of a charge $Q$ by a "calorimeter" at spatial infinity encompassing a differential solid angle $d^{(2)} \Omega$ around a direction $\hat{n}$. This corresponds to having $w_{Q}=\sum_{c} Q_{c} \delta^{(2)}\left(\hat{p}_{c}-\hat{n}_{\Omega}\right) \theta\left(p_{c}^{0}\right)$.

The cross section can also be re-written as $\sigma_{Q}(p, \hat{n}) \sim \sum_{X}\left[\left\langle\gamma^{*}(p) \mid X\right\rangle w_{Q}\left\langle X \mid \gamma^{*}(p)\right\rangle\right]$. If the factor $w_{Q}$ is replaced by a delta-function of four-momentum, $Q_{c} \delta\left(q_{c}-p_{c}\right)$, then using completeness, $\sum_{X^{\prime}}\left|X^{\prime}\right\rangle\left\langle X^{\prime}\right|=I$ where the sum over $X^{\prime}$ stands for the previous sum over $X$ with a state $\varphi_{c}$ removed. This in turn simply leads to the discontinuity of a 4-point function in the invariant $M^{2}=\left(p_{\gamma^{*}}-p_{c}\right)^{2}$. One can formally introduce $\widetilde{O}_{Q}=\sum_{c} w_{Q} \int d^{4} x e^{-i p_{c} x} \varphi_{c}(x) \varphi_{c}(0)$, leading to

$$
\sigma_{Q}(p, \hat{n})=\sum_{c} \int d^{4} p_{c} \frac{1}{2 i} w_{Q}\left(p_{c}\right) \operatorname{Disc}_{M^{2}} T_{\gamma^{*} c^{\prime} \rightarrow \gamma^{\prime *} c}
$$

in the forward limit of $p_{\gamma^{* \prime}}=p_{\gamma^{*}}$ and $p_{c}=p_{c^{\prime}}$. The discontinuity is taken for $M^{2}>0$.

The same formalism can be used to study the flow of other conserved charges, such as energy and momentum, as well as higher-point correlation functions $\left\langle\widetilde{O}_{w}(1) \widetilde{O}_{w}(2) \cdots\right\rangle$. For instance, the flow of energy in a direction $\hat{n}$ is given by $\langle E(\hat{n})\rangle=\sigma_{E}(\hat{n}) / \sigma_{\gamma^{*}}(s)$, where

$$
\sigma_{E}(\hat{n})=\sum_{c} \int d^{4} p_{c} \frac{1}{2 i} p_{c}^{0} \delta^{2}\left(\hat{p}_{c}-\hat{n}\right) \operatorname{Disc}_{M^{2}} T_{\gamma^{*} c^{\prime} \rightarrow \gamma^{\prime *} c} .
$$


This is related to the momentum space representation for the correlator $\left\langle\mathcal{O}(p)\left|\widetilde{O}_{E}\left(p_{c}\right)\right| \mathcal{O}(p)\right\rangle$; however, this is kinematically related to a position space three-point Wightman function of fields, $\left\langle\varphi_{1}(x) \varphi_{2}(y) \varphi_{3}(z)\right\rangle$. Similarly, the two-point energy correlator $\left\langle\mathcal{O}(p)\left|\widetilde{O}_{E}(1) \widetilde{O}_{E}(2)\right| \mathcal{O}(p)\right\rangle$ is related to a position-space four-point function, and so on. It is therefore desirable to explore directly conformal invariance for eq. (2.8) in a coordinate representation, as initiated in [44-47].

A similar analysis holds for higher order correlators $\left\langle\widetilde{O}_{w}(1) \widetilde{O}_{w}(2) \cdots\right\rangle$, where we now have

$$
\sigma_{w}\left(\hat{n}_{1}, \hat{n}_{2}, \cdots\right)=\sum_{c_{1}, c_{2}, \cdots} \int d^{4} p_{c_{1}} \int d^{4} p_{c_{2}} \cdots \frac{1}{2 i} w\left(p_{c_{1}}, p_{c_{2}}, \cdots\right) \operatorname{Disc}_{M^{2}} T_{\gamma^{*} c_{1}^{\prime} c_{2}^{\prime} \cdots \rightarrow \gamma^{\prime *} c_{1} c_{2} \cdots}
$$

\section{$3 \quad$ String-gauge duality}

In this section we discuss scattering via the AdS/CFT correspondence with a particular focus on scattering in the gravity theory. We first review only the essentials of scattering in AdS space needed to understand our phenomenological model and arrive to eq. (3.15). Our discussion revolves around the scattering of AdS states and stringy effects beyond the super-gravity limit of $\lambda \rightarrow \infty$. A detailed dual description in terms of the $\mathcal{N}=4$ SYM theory, while interesting and informative, is not needed for our current application. We stress that stringy effects are not only conceptually important but also phenomenologically necessary. Due to the difficulty of full finite $\lambda$ string calculations, scattering amplitudes are most easily formulated by starting with the infinite coupling limit and then calculating $1 / \sqrt{\lambda}$ corrections in the context of $1 / N_{c}$ expansion: we will treat stringy effects perturbatively.

We pay special attention to two kinematic limits where the consequences of stringy corrections can be seen easily. One limit of interest is that of fixed-angle scattering, which leads to "ultralocal" scattering in the AdS bulk and hence in the Polchinksi-Strassler regime [13]. This is briefly reviewd in appendix B. A second limit of interest is scattering in the nearforward limit which is discussed below. At high energy, the most important contribution to the AdS amplitude in this limit is due to the exchange of a graviton in the $t$-channel. However, this leads to too rapid an increase for amplitudes; stringy effects can slow the increase. In $[15,17]$ it was shown that this leads to the introduction of a "reggeized" AdS graviton known as the BPST pomeron; this pomeron serves as the leading contribution to the scattering in a unitarized treatment via an eikonal sum. This framework can also be extended to multi-particle near-forward scattering $[52,77,78]$, which paves the way for the treatment of central inclusive production in section 4 .

\subsection{AdS scattering}

The AdS/CFT correspondence relates $\mathcal{N}=4 \mathrm{SYM}$ correlation functions to a dual description in terms of correlation functions of string states in a higher-dimensional via an equivalance of partition functions. ${ }^{13}$ From the gravity perspective, CFT states can be

\footnotetext{
${ }^{13}$ The canonical description of the AdS/CFT correspondence describes string states living in $A d S_{5} \times S^{5}$. Here we are only concerned with excitations in the AdS space.
} 
thought of as propagating from a four dimensional boundary theory into the gravity bulk, scattering, and returning to the boundary CFT. In the limit of large 't Hooft coupling, this process can be described with perturbative sums of "Witten diagrams" in analogy to weak coupling descriptions. (See appendices B and C for further clarification.)

For most of the following calculations, it is sufficient to work with the Poincare patch of $A d S_{5}$, described by the metric

$$
d s_{A d S_{5}}^{2 \text { Poincare }}=\frac{z^{2}}{R^{2}}\left\{-d t^{2}+d \vec{x}^{2}+d z^{2}\right\},
$$

where $R$ is the AdS radius. This metric corresponds to a boundary theory with purely conformal dynamics, as can be seen by comparing the five-dimensional AdS isometry group to the four-dimensional conformal group. The radius $R$ of the bulk geometry is related to the 't Hooft coupling $\lambda \equiv g_{Y M}^{2} N_{c}$ of the boundary gauge theory by $\lambda=\left(R / \ell_{\text {string }}\right)^{4}$, where $\ell_{\text {string }}=\sqrt{\alpha^{\prime}}$ is the string length. Therefore, the limit $\lambda \rightarrow \infty$ of strong boundary coupling corresponds to a weakly curved bulk geometry, and hence weakly coupled bulk dynamics. In these coordinates, $z \rightarrow 0$ and $z \rightarrow \infty$ correspond to the UV and IR of the dual gauge theory, respectively.

However, we will also be interested in deforming away from a strictly conformal boundary limit, by introducing a confinement scale in the boundary theory. There are a variety of approaches to introducing a confinement deformation in AdS space [79-88], but we are interested in universal features that are common to all the approaches. Generically, a confining gauge theory has a bulk dual with metric

$$
d s^{2}=e^{A(z)}\left\{-d t^{2}+d \vec{x}^{2}+d z^{2}\right\},
$$

where $A(z)$ describes both the AdS warping and the deformation away from pure AdS. Sometimes, as in the so-called "hard wall" models of QCD, the coordinates are restricted to lie in finite intervals. The presence of a confinement deformation introduces a new length scale $\Lambda^{-1} \gg R$; we take $\Lambda \sim \Lambda_{Q C D}$.

For concreteness, in most of the rest of the discussion we will assume a hard wall deformation, where we put in a hard IR cutoff by restricting the AdS radial coordinate $z$ to lie in the interval $\left[0, z_{\max }\right]$. Then the confinement scale $\Lambda$ is given by $\Lambda \sim z_{\max }^{-1}$. However, we expect our main results to be essentially independent of exactly which confinement deformation is used, since they depend essentially on the conformal UV dynamics.

A connected Green's function $\tilde{G}_{F}$ in the boundary theory can now be expressed in terms of an amplitude in the AdS bulk via a convolution

$$
\tilde{G}_{F}\left(p_{1}, p_{2}, \cdots\right)=\int \cdots \int \Pi_{i}\left\{d \mu\left(z_{i}\right) \mathcal{G}_{i}\left(p_{i}, z_{i}\right)\right\} \mathcal{T}_{n}\left(p_{1}, z_{1}, p_{2}, z_{2}, \cdots\right),
$$

where $d \mu(z)=d z \sqrt{-g}$ and $g=\operatorname{det} g . \mathcal{T}_{n}$ can be considered as an "amputated Green's function", and $\mathcal{G}(p, z)$ is the bulk-to-boundary propagator, which, for a scalar of conformal dimension $\Delta$, is given up to a normalization factor in terms of Bessel function of the second kind,

$$
\mathcal{G}(p, z)=z^{2} K_{\Delta-2}\left(z \sqrt{-p^{2}}\right)=z^{-\Delta} \int_{0}^{\infty} d x x^{\Delta-1} \frac{J_{\Delta-2}(x)}{x^{2}-z^{2} p^{2}}
$$


We will not provide here a detailed discussion on the Witten diagram expansion here except for several remarks, which will become relevant shortly.

Consider first the bulk-to-bulk Feynman propagator $\left\langle 0\left|\mathbf{T}\left\{\varphi(x, z) \varphi\left(x^{\prime}, z^{\prime}\right)\right\}\right| 0\right\rangle$ of a scalar with conformal dimension $\Delta$. Its momentum representation, which will be designated as $G_{F}\left(z, z^{\prime}, p_{\mu}\right)$, can again be expressed in terms of Bessel functions as

$$
G_{F}\left(z, z^{\prime}, p^{2}\right)=\left(z z^{\prime}\right)^{2} \int_{0}^{\infty} k d k \frac{J_{\Delta-2}(k z) J_{\Delta-2}\left(k z^{\prime}\right)}{k^{2}-p^{2}} .
$$

Since there is no mass gap, $G_{F}\left(z, z^{\prime}, p^{2}\right)$ is analytic in $p^{2}$, with a branch cut over $0 \leq$ $p^{2}<\infty$. Its discontinuity over the branch cut, which corresponds to the momentum-space representation for the Wightman function $G_{W}\left(x, z ; x^{\prime}, z^{\prime}\right)=\left\langle 0\left|\varphi\left(x^{\prime} z^{\prime}\right) \varphi(x, z)\right| 0\right\rangle$, is

$$
G_{W}\left(z, z^{\prime}, p^{2}\right)=\frac{\pi}{2}\left(z z^{\prime}\right)^{2} J_{\Delta-2}(p z) J_{\Delta-2}\left(p z^{\prime}\right) \theta\left(p^{2}\right) .
$$

In the limit of $z, z^{\prime} \rightarrow 0$, it approaches, up to a normalization constant, the Wightman function in eq. (2.4).

Confinement deformation in the IR, universality and conformal invariance. Let us return to the issue of on-shell amplitudes. For CFTs, associated with each leg of the Green's function $G_{n}$ is an off-shell wave-function, $e^{i p_{\mu} x^{\mu}}$, and a bulk-to-boundary propagator, $\left.G\left(x^{\prime}, z^{\prime} ; z, x\right)\right|_{z^{\prime} \rightarrow 0}$. In order to define on-shell amplitudes, it is necessary to introduce a confinement deformation in the IR leading to finite a mass gap. A new dimensionful scale, $\Lambda^{-1} \gg R$, enters serves as the basic length scale. Conformality holds for $z \ll \Lambda^{-1}$. Conversely, confinement effect becomes important if $z \sim \Lambda^{-1}$, with $\Lambda$ expected to be of the order $\Lambda_{Q C D}$.

In such a scenario, on-shell amplitudes are given by amputated Green's functions, which have a normal singularity structure as in standard flat space field theories. After the introduction of a confinement deformation in the IR, the spectrum of the bulk theory becomes discrete, so that the propagator in eq. (3.5) is replaced by a discrete sum,

$$
G_{F}\left(z, z^{\prime}, p^{2}\right) \rightarrow \sum_{n} \frac{\varphi_{n}(z) \varphi_{n}\left(z^{\prime}\right)}{m_{n}^{2}-p^{2}}
$$

where the $\varphi_{n}(z)$ are a set of orthonormal wave functions associated with an infinite set of scalar glueballs of increasing mass $m_{n}{ }^{14}$ More importantly, the bulk-to-boundary propagator in eq. (3.4) is also given by a discrete sum,

$$
\mathcal{G}(p, z) \rightarrow \sum_{n} \frac{c_{n} \varphi_{n}(z)}{m_{n}^{2}-p^{2}}
$$

with poles at $p^{2}=m_{n}^{2}$. This in turns allows us to extract on-shell amplitudes in a standard manner.

Although our discussion will turn to theories with an IR confinement deformation, there are features of the Witten diagram expansion that are model independent. As stressed

\footnotetext{
${ }^{14}$ These states also interpolate with higher spin glueball states on the same Regge trajectories, leading to the reggeized $J$-dependent propagator appearing in eq. (B.10).
} 


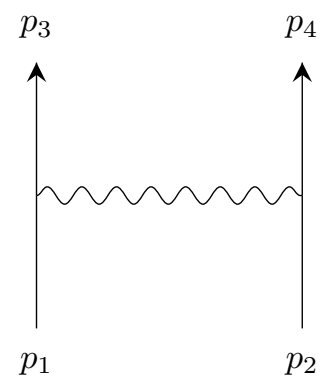

Figure 1. Schematic representation of high-energy elastic two-to-two scattering. The internal line represents the Pomeron kernel defined in eq. (3.12).

in $[15,17]$, it is possible to identify features which depend only on the conformal structure, such as the large $Q^{2}$ behavior of DIS at small- $x$. We stress here the important fact that AdS wave functions have universal behavior ${ }^{15}$ in the UV. As $z \rightarrow 0$,

$$
\varphi_{i}(z) \simeq z^{\tau_{i}}, \quad \tau_{i}=\Delta_{i}-J_{i}
$$

where $\tau$ is the twist and $J$ is the spin. This behavior is independent of the confinement deformation and depends only on the conformal properties. We shall make use of this fact when implementing the Polchinski-Strassler mechanism for large $p_{\perp}$ production.

It is now possible to define scattering amplitudes as amputated Green's functions by going on to the pole for each external state, leading to on-shell scattering amplitudes,

$$
T_{n}\left(p_{1}, p_{2}, \cdots\right)=\int \cdots \int \Pi_{i}\left\{d \mu\left(z_{i}\right) \varphi_{i}\left(z_{i}\right)\right\} \mathcal{T}_{n}\left(p_{1}, z_{1}, p_{2}, z_{2}, \cdots\right) .
$$

For each external on-shell particle, one associates a bulk wave-function $e^{-i p x} \varphi(z)$. This can also be extended to multi-particle inclusive productions which we will turn to shortly.

High energy limit. In this paper, we will be primarily be interested in inclusive processes due to scattering at high energies where the source is in general non-local. One therefore will deal with $(2 n)$-point functions for $n=2,3, \cdots$. It is interesting to note that non-trivial dynamics already occur at the lowest level, for example the $\gamma^{*} p$ total cross section [27-29]. More generally, an inclusive discontinuity can be taken through Witten diagrams in a momentum representation. This can be done most readily for near forward scattering at high energy in the Regge limit.

There exists a rather extensive literature on the applications of AdS/CFT to high energy near-forward scattering [16-18, 24, 25, 28, 51]. The factorization of AdS amplitudes has emerged as a universal feature, present in the scattering of both particles and currents. The amplitude for elastic two-to-two scattering can be represented schematically in a factorized form as

$$
T_{2 \rightarrow 2}=\Phi_{13} * \widetilde{\mathcal{K}}_{P} * \Phi_{24}
$$

\footnotetext{
${ }^{15}$ In the hard wall model, the glueball wave function has $\varphi(z) \propto z^{2} J_{\Delta-2}\left(m_{n} z\right) \sim z^{\Delta}$ as $z \rightarrow 0$. A similar explicit analytic expression can also be obtained for other deformations, such as the "soft wall" model.
} 
where $\Phi_{13}$ and $\Phi_{24}$ are elastic vertices and the convolution, $*$, involves an integration of the vertex position over the AdS bulk, as in eqs. (B.2)-(B.3). This can be seen in figure 1. The Pomeron kernel $\widetilde{\mathcal{K}}_{P}$, described in more detail in appendix $\mathrm{B}$, is defined as

$$
\widetilde{\mathcal{K}}_{P}\left(s, t, z, z^{\prime}\right)=-\int_{L-i \infty}^{L+i \infty} \frac{d j}{2 \pi i}\left(\alpha^{\prime} \widetilde{s}\right)^{j} \frac{1+e^{-i \pi j}}{\sin \pi j} \widetilde{G}_{j}\left(t, z, z^{\prime}\right),
$$

where the reggeized graviton propagator $\widetilde{G}_{j}\left(t, z, z^{\prime}\right)$ is defined in eq. (B.8).

Through AdS/CFT, one can identify the Pomeron with a reggeized graviton in the AdS bulk. The Pomeron kernel $\widetilde{\mathcal{K}}_{P}$ can be introduced by perturbing about the supergravity limit through a world-sheet OPE. More formally, one can introduce a Pomeron vertex operator in AdS, as done in [15], so that

$$
T_{2 \rightarrow 2}=\left\langle\varphi_{1} \varphi_{3} \mid \mathcal{V}_{P}\right\rangle \Pi\left(\alpha_{P}\right) s^{\alpha_{P}}\left\langle\mathcal{V}_{P} \mid \varphi_{2} \varphi_{4}\right\rangle
$$

where $\Pi\left(\alpha_{P}\right)$ is a complex "signature factor" carrying information about its phase that is useful for taking the discontinuity. It is customary to normalize this signature factor as $\Pi(j)=\frac{1+e^{-i \pi j}}{\sin \pi j}$ so that $\operatorname{Im} \Pi(j)=1$.

\subsection{Inclusive cross sections as AdS discontinuities}

In eq. (3.10), we have expressed on-shell scattering amplitudes $T_{n}$ in the boundary theory in terms of scattering amplitudes $\mathcal{T}_{4}\left(p_{1}, z_{1}, \cdots\right)$ in the AdS bulk. We can now extend this treatment to inclusive cross sections. After applying eq. (1.3) to eq. (3.10) for $n=4$, we find that the cross section for $a+b \rightarrow X$ is given in terms of a bulk amplitude as

$$
\sigma_{\text {total }}=(1 / s) \int\left\{\Pi_{i=1-4} d \mu\left(z_{i}\right) \varphi_{n}\left(z_{i}\right)\right\} \operatorname{Im} \mathcal{T}_{4}\left(p_{1}, z_{1}, \cdots\right) .
$$

Similarly, by applying eq. (2.7) to eq. (3.10) for $n=6$, we find that the differential inclusive cross section for $a+b \rightarrow c+X$ is given by

$$
\frac{d \sigma_{a b \rightarrow c+X}}{d^{3} \mathbf{p}_{c} / E_{c}} \simeq \frac{1}{2 i s} \int\left\{\Pi_{i=1-6} d \mu\left(z_{i}\right) \varphi_{n}\left(z_{i}\right)\right\} \operatorname{Disc}_{M^{2}>0}\left\{\mathcal{T}_{a b c^{\prime} \rightarrow a^{\prime} b^{\prime} c}\left(p_{i}, z_{i}\right)\right\}
$$

Both of these discontinuities are taken in appropriate forward limits.

This key result can also be extended to multi-particle inclusive production. As an explicit illustration, consider the case of DIS. Here one first replaces $\Phi_{13}$ in eq. (3.11) by the appropriate product of propagators for external currents [26, 27]. One next performs the step of taking discontinuity [27-32], leading to a factorized form for the cross section:

$$
\sigma_{\gamma^{*} p}^{\text {total }} \simeq \frac{1}{s} \Phi_{13} *\left[\operatorname{Im} \widetilde{\mathcal{K}}_{P}\right] * \Phi_{24} .
$$

For the general two-to-two scattering of scalar glueballs, $1+2 \rightarrow 3+4$, one has

$$
\operatorname{Im} T_{2 \rightarrow 2}(s, t=0)=\int d \mu(z) \int d \mu\left(z^{\prime}\right) \Phi_{13}(z) \operatorname{Im} \widetilde{\mathcal{K}}_{P}\left(\widetilde{s}, 0, z, z^{\prime}\right) \Phi_{24}\left(z^{\prime}\right),
$$

where the vertex coupling $\Phi_{a b}(z)$ involves the normalized wave-function $\varphi_{a}(z)$ of scalar glueball of conformal dimension $\Delta$. As indicated earlier, for a hard-wall deformation, we 
have $\varphi_{a}(z) \sim z^{2} J_{(\Delta-2)}\left(m_{a} z\right)$ and $\int d \mu(z) z^{2} \varphi_{a}(z) \varphi_{b}(z)=\delta_{a, b}$. Similarly, the reggeized Pomeron kernel $\widetilde{\mathcal{K}}_{P}$ in eq. (C.5) can be given a more explicit form in the hard wall model $[15,89]$. We will not discuss this propagator in detail, except to note that its phase information is given by eq. (3.13). The propagator has a discontinuity in $\widetilde{s}$, with its leading behavior given by

$$
\operatorname{Disc}_{s} \widetilde{\mathcal{K}}_{P}\left(\widetilde{s}, 0, z, z^{\prime}\right) \propto \widetilde{s}^{j_{0}},
$$

with $j_{0} \simeq 2-2 / \sqrt{\lambda}$. In the particular case of DIS, this leads to eq. (A.3), with anomalous dimensions for $j \simeq 2$ at strong coupling given by

$$
\gamma(j)=\sqrt{2 \sqrt{\lambda}\left(j-j_{0}\right)}-j+O\left(\lambda^{-3 / 4}\right) .
$$

The results of this section rely on identifying that the analytic structure of amplitudes in AdS/CFT are analogous to that of Field Theory. However, in the next section we turn our attention to a specific high energy process appropriate for collider scattering. Here, collisions with large transverse momentum will be localized in a transverse space and in the Polchinski-Strassler regime; we can consider flat-space string vertices with physical momenta red shifted by the geometry. This is more fully explored in $[15,52]$ and analogous situations for specific collider physics are described in [24, 25, 27, 28, 30, 77, 78, 90, 91].

\section{Inclusive single-particle production in the central region}

We have shown that the inclusive single particle production cross section in the boundary theory can be related to the discontinuity of the six-point amplitude in the bulk. In order to evaluate this discontinuity, we must generalize the treatment of two-to-two amplitudes given above to apply to three-to-three amplitudes. We begin by discussing the kinematics of inclusive production.

For fixed $X$, the inclusive processes $a+b \rightarrow c+X$ can be treated kinematically as a two-to-two process where we treat $X$ effectively as a particle with mass

$$
M^{2}=\left(p_{a}+p_{b}-p_{x}\right)^{2} .
$$

Thus, in addition to $M^{2}$, we have the usual three Mandelstam invariants

$$
s=\left(p_{a}+p_{b}\right)^{2}, \quad t=\left(p_{a}-p_{c}\right)^{2}, \quad u=\left(p_{b}-p_{c}\right)^{2} .
$$

These invariants are related by the constraint

$$
M^{2}=s+t+u-m_{a}^{2}-m_{b}^{2}-m_{c}^{2} .
$$

Therefore, the kinematics can be parameterized by three invariants, which can be taken to be $\left(s, t, M^{2}\right){ }^{16}$

However, there exists an alternate parameterization that can better illuminate the simplicity of the actual process. A universal characteristic of high energy particle production

\footnotetext{
${ }^{16}$ For central production, it turns out to be more convenient to use $\left(M^{2}, t, u\right)$ as independent variables, as explained below.
} 
is the fact that the majority of produced particles will have small transverse momentum relative to the (longitudinal) incoming direction. In a typical hadronic collision at the LHC, the detector essentially sits at rest in the center of momentum frame of the two incoming particles, which have equal and opposite large momentum; these momenta define a longitudinal light cone (LC) direction. To be more explicit, we choose the incoming particles $a$ and $b$ to have LC momenta $p_{a}=\left(p_{a}^{+}, p_{a}^{-}, \vec{p}_{\perp, a}\right)=\left(m_{a} e^{Y / 2}, m_{a} e^{-Y / 2}, 0\right)$ and $p_{b}=\left(p_{b}^{+}, p_{b}^{-}, \vec{p}_{\perp, b}\right)=\left(m_{b} e^{-Y / 2}, m_{b} e^{Y / 2}, 0\right)$, where $Y$ is the rapidity. Then, taking $m_{a}=m_{b}=m$ for simplicity, the Mandelstam $s$ invariant is given by $s \sim m^{2} e^{Y}$, and the produced particle has LC momentum given by

$$
p_{c}=\left(m_{\perp} e^{y}, m_{\perp} e^{-y}, \vec{p}_{\perp}\right), \quad m_{\perp}^{2} \equiv m_{c}^{2}+\vec{p}_{\perp}^{2} .
$$

Equivalently, the produced particle has energy $E=m_{\perp} \cosh y$ and longitudinal momentum $p_{L}=m_{\perp} \sinh y$ so that $y=\ln \left[\left(E+p_{L}\right) /\left(E-p_{L}\right)\right]$.

Inclusive central production involves particles with fixed $\vec{p}_{\perp}$ and $y$ in the CM frame in the $s \rightarrow \infty$ limit, and therefore incoming particles have large rapidities, $-y_{b} \simeq y_{a}=$ $Y \rightarrow \infty$. In such an event, the produced particles can be grouped in an intuitively helpful way as $a+b \rightarrow X_{1}+c+X_{2}$, where $c$ is the centrally produced particle and $X_{1}$ and $X_{2}$ are left- and right-moving particles, respectively. In this limit, the traditional Mandelstam variables behave as

$$
\begin{aligned}
& s \simeq M^{2} \simeq m^{2} e^{Y} \rightarrow+\infty \\
& t \simeq-m m_{\perp} e^{Y / 2-y} \rightarrow-\infty \\
& u \simeq-m m_{\perp} e^{Y / 2+y} \rightarrow-\infty .
\end{aligned}
$$

We can additionally check that the ratio

$$
\kappa \equiv \frac{(-t)(-u)}{M^{2}} \simeq m_{\perp}^{2}=m_{c}^{2}+p_{\perp}^{2}
$$

is fixed. These kinematic conditions can be thought of as the definition of central production. Phenomenologically, we often prefer to use $\left(s, y, p_{\perp}^{2}\right)$ as the three independent variables describing the kinematics of central production at the LHC. On the other hand, when we take the discontinuity in the 3 -to-3 amplitude, we will see that it is more convenient to parameterize the kinematics with $\left(M^{2}, t, u\right)$, and to therefore treat $s$ as a dependent variable. We will return to this issue shortly.

\subsection{Inclusive central production and the 3-to-3 amplitude}

A holographic analysis of the 2-to-3 amplitude in the double Regge limit was performed in [52] by generalizing the AdS treatment of 2-to-2 scattering. Schematically, this 2-to-3 bulk amplitude can be represented by

$$
T_{2 \rightarrow 3}=\Phi_{13} * \widetilde{\mathcal{K}}_{P} * V_{c} * \widetilde{\mathcal{K}}_{P} * \Phi_{24},
$$

where we have introduced a new 3-point central production vertex, $V_{c}$, shown in figure 2. In terms of the Pomeron vertex operator, $V_{c}$ can be expressed as $V_{c}=\left\langle\mathcal{V}_{P}\left|\varphi_{c}\right| \mathcal{V}_{P}\right\rangle$. These AdS 


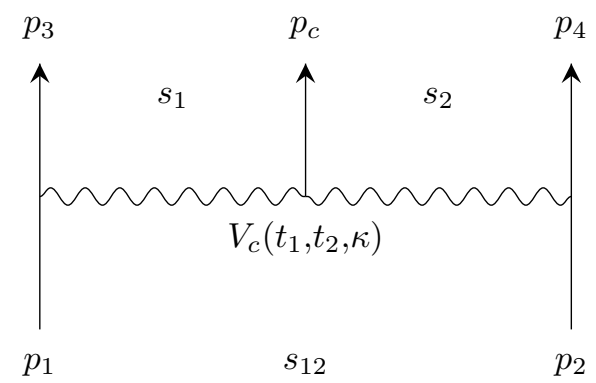

Figure 2. A schematic representation of the factorized two-to-three amplitude in eq. (4.7).

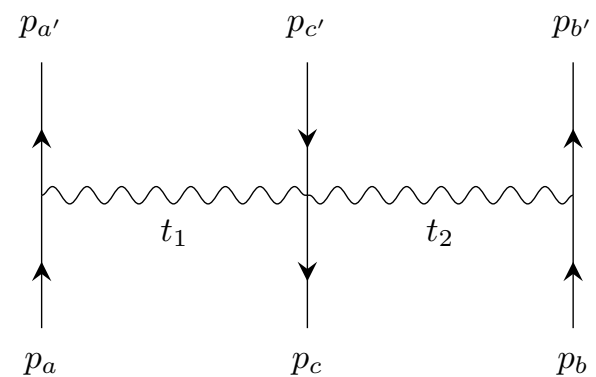

Figure 3. A schematic representation of the factorized three-to-three amplitude in eq. (4.8).

vertex operators are closed string operators where the invariants are redshifted. In general these can be complicated expressions. However, following the analysis [24, 25, 52, 77, 78], many of the general features are shared with the much simpler flat space string theory vertex operators which we review in appendix D.

We now move on to the six-point function, which was discussed for flat-space string scattering in [56-58]. Following the above discussion and the logic in [52, 77, 78], we will be interested in the limit where the three-to-three amplitude takes on a factorized form, given by

$$
T_{a b c^{\prime} \rightarrow a^{\prime} b^{\prime} c}=\Phi_{13} * \widetilde{\mathcal{K}}_{P} * V_{c \bar{c}} * \widetilde{\mathcal{K}}_{P} * \Phi_{24} .
$$

Again we have had to introduce a new central vertex, $V_{c \bar{c}}$, shown in figure 3 , which can formally be expressed as the matrix element involving two pomeron vertex operators

$$
V_{c \bar{c}}\left(\widetilde{\kappa}, \widetilde{t}_{1}, \widetilde{t}_{2}\right)=\left\langle\mathcal{V}_{P}\left|\varphi_{c} \varphi_{\bar{c}}\right| \mathcal{V}_{P}\right\rangle
$$

Following the flat space calculation in [56-58], we can take the $M^{2}$ discontinuity in the amplitude to find that [92]

$$
(1 / 2 i) \operatorname{Disc}_{M^{2}} T_{a b c^{\prime} \rightarrow a^{\prime} b^{\prime} c}=\Phi_{13} *\left[\operatorname{Im} \widetilde{\mathcal{K}}_{P}\right] *\left[\operatorname{Im} V_{c \bar{c}}\right] *\left[\operatorname{Im} \widetilde{\mathcal{K}}_{P}\right] * \Phi_{24} .
$$

As in two-to-three scattering, this AdS-space central vertex $V_{c \bar{c}}\left(\tilde{\kappa}, \tilde{t}_{1}, \tilde{t}_{2}\right)$ has the same functional form as the flat space vertex,

$$
\mathcal{V}_{c \bar{c}}=\int_{0}^{1} \frac{d y}{y^{\alpha_{\bar{a} a \bar{c}}+1}(1-y)^{\alpha_{b \bar{b} c}+1}} V_{c}\left(t_{1}, t_{2}, \frac{\alpha^{\prime} \kappa}{y(1-y)}\right),
$$


but with the arguments appropriately redshifted. (We follow here notation of [56]. See appendix $\mathrm{D}$ for more details.) The invariant $\kappa$ was defined in eq. (4.6), and can also be expressed as

$$
\kappa \simeq \frac{(-t)(-u)}{M^{2}}
$$

where $t \equiv s_{1}<0$ and $u \equiv s_{2}<0$ and $M^{2}$ are defined in eqs. (4.2) and (4.3). The singularity of $T_{a b c^{\prime} \rightarrow a^{\prime} b^{\prime} c}$ in $M^{2}$ now appears only as a singularity of $V_{c \bar{c}}$ in $\kappa$, with discontinuity given by

$$
\operatorname{Im} \mathcal{V}_{c \bar{c}}\left(\kappa, t_{1}, t_{2}\right)=\int_{0}^{1} \frac{d y}{y^{\alpha_{\bar{a} a \bar{c}}+1}(1-y)^{\alpha_{b \bar{b} c}+1}} \operatorname{Im} V_{c}\left(t_{1}, t_{2}, \frac{\alpha^{\prime} \kappa}{y(1-y)}\right) .
$$

At $t_{1}=t_{2}=0, \alpha_{\bar{a} a \bar{c}}(0)=\alpha_{b \bar{b} c}(0)=0$, for external tachyons, with $\operatorname{Im} \mathcal{V}_{c \bar{c}}(\kappa, 0,0)$ finite.

We can now explicitly write out the bulk six-point amplitude. Putting everything together, eq. (4.8) can be expressed as

$$
\begin{aligned}
& T_{a b c^{\prime} \rightarrow a^{\prime} b^{\prime} c}\left(\kappa, s_{1}, s_{2}, t_{1}, t_{2}\right) \\
& =\frac{g_{0}^{2}}{R^{4}} \int_{0}^{z_{\max }} d z_{1} \sqrt{\left|g\left(z_{1}\right)\right|}\left[z_{1}^{2} \varphi_{a}\left(z_{1}\right) \varphi_{a^{\prime}}\left(z_{1}\right)\right] \int_{0}^{z_{\max }} d z_{2} \sqrt{\left|g\left(z_{2}\right)\right|}\left[z_{2}^{2} \varphi_{b^{\prime}}\left(z_{2}\right) \varphi_{b}\left(z_{2}\right)\right] \\
& \quad \times \int_{0}^{z_{\max }} d z_{3} \sqrt{\left|g\left(z_{3}\right)\right|} \widetilde{\mathcal{K}}_{P}\left(-\tilde{s}_{1}, \tilde{t}_{1}, z_{1}, z_{3}\right) I\left(\tilde{\kappa}, \tilde{t}_{1}, \tilde{t}_{2}, z_{3}\right) \widetilde{\mathcal{K}}_{P}\left(-\tilde{s}_{2}, \tilde{t}_{2}, z_{2}, z_{3}\right), \quad
\end{aligned}
$$

where the dependence on the central vertex is collected as

$$
I\left(\tilde{\kappa}, \tilde{t}_{1}, \tilde{t}_{2}, z_{3}\right)=\left(z_{3}^{2} \varphi_{c}\left(z_{3}\right)\right) V_{c \bar{c}}\left(\tilde{\kappa}, \tilde{t}_{1}, \tilde{t}_{2}\right)\left(z_{3}^{2} \varphi_{c^{\prime}}\left(z_{3}\right)\right) .
$$

In eq. (4.14), we have also introduced an explicit IR cutoff, $z_{\max }$, which should be of the order $O\left(\Lambda_{Q C D}^{-1}\right)$; this amounts to implementing a hard wall confinement deformation. It is essential that all Mandelstam invariants in this amplitude are holographic quantities, related to the flat space invariants by the prescription in eq. (B.4). For instance, $\tilde{s}_{1}<0$ and $\tilde{s}_{2}<0$ are given by $\tilde{s}_{1}=\left(\tilde{p}_{a}-\tilde{p}_{c}\right)^{2}=\left(\frac{z_{1}}{R} p_{a}-\frac{z_{3}}{R} p_{c}\right)^{2} \sim \frac{z_{1} z_{3}}{R^{2}} s_{1}<0$ and $\tilde{s}_{2}=\left(\tilde{p}_{b}-\tilde{p}_{c}\right)^{2}=$ $\left(\frac{z_{1}}{R} p_{b}-\frac{z_{3}}{R} p_{c}\right)^{2} \sim \frac{z_{1} z_{3}}{R^{2}} s_{2}<0$. Other important holographic invariants are

$$
\tilde{M}^{2}=\left(\tilde{p}_{a}+\tilde{p}_{b}-\tilde{p}_{c}\right)^{2} \sim \frac{z_{1} z_{2}}{R^{2}} M^{2}, \quad \text { and } \quad \tilde{\kappa}=\frac{\tilde{s}_{1} \tilde{s}_{2}}{\tilde{M}^{2}} \sim \frac{z_{3}^{2}}{R^{2}} \frac{s_{1} s_{2}}{M^{2}}=\frac{z_{3}^{2}}{R^{2}} \kappa .
$$

In this limit, we have $s \simeq M^{2} \gg\left|s_{1}\right|,\left|s_{2}\right|$.

Next we will compute the discontinuity in the missing mass $M^{2}$, given in eq. (4.10), in the forward limit. From eq. (4.10), we see that, due to factorization, eq. (4.8), as schematically represented by figure 3 , the discontinuities $\left[\operatorname{Im} \widetilde{\mathcal{K}}_{P}\left(-\tilde{s}_{1}, 0, z_{1}, z_{3}\right)\right]$ and $\left[\operatorname{Im} \widetilde{\mathcal{K}}_{P}\left(-\tilde{s}_{2}, 0, z_{2}, z_{3}\right)\right]$ lead to the $z_{3}$ integral being entirely independent of $z_{1}$ and $z_{2}$. (See eq. (B.9).) Thus, we can perform the $z_{1}$ and $z_{2}$ integrals to find an inclusive particle density $\rho$ for central production given by

$$
\begin{aligned}
\rho\left(\vec{p}_{T}, y, s\right) & \equiv \frac{1}{\sigma_{\text {total }}} \frac{d^{3} \sigma_{a b \rightarrow c+X}}{d \mathbf{p}_{c}^{3} / E}=\frac{1}{2 i s \sigma_{\text {total }}(s)} \operatorname{Disc}_{M^{2}} T_{6}\left(\kappa, s_{1}, s_{2}, 0,0\right) \\
& =\beta \int_{0}^{z_{\max }} \frac{d z_{3}}{z_{3}} \tilde{\kappa}^{j_{0}}\left[\varphi_{c}\left(z_{3}\right)\right]^{2}\left[\operatorname{Im} \mathcal{V}_{c \bar{c}}(\tilde{\kappa}, 0,0)\right],
\end{aligned}
$$

where $\beta$ is an overall constant partially stemming from the $z_{1}$ and $z_{2}$ integrals. This is our key result. 


\subsection{Central production at large $p_{\perp}$ and conformal invariance}

It should be stressed that eq. (4.17) depends crucially on factorization in the double-Regge limit. In the factorization limit, the particle density is independent of both $y$ and $s .{ }^{17}$ Conversely, the density depends on $p_{\perp}$ through the wavefunction $\varphi_{c}(z)$ and the vertex $\operatorname{Im} \mathcal{V}_{c \bar{c}}\left(z^{2} \kappa / R^{2}, 0,0\right)$. Recall that the double Regge kinematics are such that $\kappa \simeq p_{\perp}^{2}+m_{c}^{2}$, and therefore that taking $p_{\perp}$ large is equivalent to working in the limit where $\kappa$ is large. We can then check that conformal dynamics emerge in this limit, as we saw above in the fixed-angle limit.

In flat space string scattering, the six-point central vertex $\mathcal{V}_{c \bar{c}}(\kappa, 0,0)$ is an analytic function of $\kappa$, away from a branch cut along the positive real line. In the limit $\kappa \rightarrow \infty$, the discontinuity vanishes and the vertex becomes factorizable with an exponentially small imaginary part: $\operatorname{Im} \mathcal{V}_{c \bar{c}}$ decays exponentially. From eqs. (4.13) and (D.12), we have, for large $\kappa$,

$$
\operatorname{Im} \mathcal{V}_{c \bar{c}}(\kappa, 0,0) \simeq \pi\left(\alpha^{\prime} \kappa\right) \int_{0}^{1} d y e^{-\frac{\alpha^{\prime} \kappa}{y(1-y)}} \sim \sqrt{\alpha^{\prime} \kappa} e^{-4 \alpha^{\prime} \kappa}
$$

This parallels the result for exclusive fixed-angle scattering in eq. (B.6). As emphasized in [56-58], this exponential suppression reflects the "softness" of flat-space string scattering.

When the scattering occurs on an AdS background, the large $\kappa$ asymptotics are rather different. The redshifted vertex is now

$$
\mathcal{V}_{c \bar{c}}(\widetilde{\kappa}, 0,0) \sim e^{-2 \alpha^{\prime} \kappa z^{2} / R^{2}} \sim e^{-2\left(z^{2} / \sqrt{\lambda}\right) \kappa}
$$

where we have substituted $\alpha^{\prime} \rightarrow \frac{1}{2} \alpha^{\prime}$ to return to closed string scattering. Thus, the $z_{3}$ integrand picks up an exponential suppression for large $z_{3}$. This induces an effective cutoff $z_{s}$. We determine $z_{s}$ by demanding $2 \alpha^{\prime} \widetilde{\kappa}=O(1)$, so that

$$
z_{s} \sim \frac{R}{\sqrt{2 \alpha^{\prime} \kappa}}=\frac{\lambda^{1 / 4}}{\sqrt{2 \kappa}} .
$$

We can thus approximate eq. (4.14) by integrating only up to $z_{3}=z_{s} \ll z_{\max }$, where the exponential factor is of order one and can be neglected. Additionally, since we are taking $\kappa \rightarrow \infty$, we can, following eq. (3.9), approximate each wave-function by $\varphi(z) \simeq z^{\tau}$, where $\tau$ is the twist. Thus eq. (4.17) becomes

$$
\begin{aligned}
\frac{1}{\sigma_{\text {total }}} \frac{d^{3} \sigma_{a b \rightarrow c+X}}{d \mathbf{p}_{c}^{3} / E_{c}} & =\beta \int_{0}^{z_{s}} \frac{d z}{z} z^{2 \tau_{c}}\left(\kappa z^{2} / R^{2}\right)^{j_{0}} e^{-\left(2 \kappa / \lambda^{1 / 2}\right) z^{2}} \\
& \simeq \beta^{\prime} \kappa^{-\tau_{c}}
\end{aligned}
$$

where we have introduced a new normalization constant $\beta^{\prime}$. In the simplest model of bulk physics, the external particles labeled by $c$ are scalar glueballs and thus have $\tau_{c}=\Delta_{c}=4$. We therefore have

$$
\rho\left(p_{\perp}, y, s\right)=\frac{1}{\sigma_{\text {total }}} \frac{d^{3} \sigma_{a b \rightarrow X}}{d \mathbf{p}_{\perp}^{2} d y} \sim p_{\perp}^{-8}
$$

\footnotetext{
${ }^{17}$ Saturation effects can cause dependence on these kinematics, which will be discussed briefly in section 6 .
} 
This result follows essentially from conformality, since it depends on the behavior of the external wave functions away from the confinement region; our prediction does not depend on the details of the confinement deformation chosen. It serves as a generalized scaling law for inclusive distribution, as is the case for exclusive fixed-angle scattering [73-75].

\section{Evidence for conformality}

We have argued that conformal symmetry is manifested in the presence of power law behavior in inclusive scattering processes. We will now test this prediction by direct comparison to experimental results. We will focus on differential cross section measurements at high $\sqrt{s}$ performed at the LHC. Many recent measurements are in the form of a double differential cross section, in which particle production is binned both in the transverse momentum $p_{T}$

and the pseudorapidity $\eta$; symbolically, these studies measure the cross section $\frac{1}{2 \pi p_{T}} \frac{d^{2} \sigma}{d p_{\mathrm{T}} d \eta}$. Here we are interested in the region where $p_{T}>\Lambda_{Q C D}$ where $y \approx \eta$. In principle, this is not precisely the quantity we have computed above. However, as discussed in [93], these two cross sections encode essentially the same information, so we expect essentially the same dependence on the kinematic variables. More concretely, we expect that the leading order physics should be independent of $\eta$, and that the exponent of the power law should be independent of $s$.

Our goal is to fit conformally motivated behavior to differential cross sections. We will use our central results, eq. (4.17)-(4.20), to model p-p [93, 94] and p-pb [95] central production via eq. (4.22). One of our assumptions from section 3.2 going into eq. (4.22) is that the incident wave functions behave as $\varphi_{a, b}(z) \approx z^{2} J_{(\Delta-2)}\left(m_{a, b} z\right)$. This is consistent with hard and soft wall AdS confinement schemes where the wave function scale has been shown to be $m_{a, b} \approx 1 \mathrm{GeV}$, or the size of a proton. [27, 28, 30, 77, 78, 90, 91] Although no heavy ion studies have been done, we assume a similar wave function form holds for $\mathrm{pb}$ as well. As described in section 4.2, the simplest model of bulk physics describes the production wave function, $\rho_{c}$, to be that of scalar glueballs which will hadronize into the detected charged particles.

As briefly described in [93], the central production of charged particles in pp and pb collisions is inherently non perturbative. Described by the kinematics of section 4 , the inclusive central production is described via a color-singlet exchange (Pomeron) which dominates in the Regge limit. The only current Monte Carlo (MC) methods used to describe this data involve a combination of multi-parton interactions involving single and double diffractive dissociation (including Pomeron and gluon effects), Gribov-Regge theory, and a "semi-hard" Pomeron model. In this kinematical region, these MC methods agree on a description of the differential cross section, but vary in describing event track multiplicities and mean transverse momentum distributions. For the p-pb collisions there is no current MC prediction.

At large $p_{T}$ our result implies that the differential cross section is described by the exchange of Reggeized objects leading to power law behavior depending on conformal dimensions. However, this behavior is only expected to hold at moderately high $p_{\mathrm{T}}$ above the QCD scale. At low $p_{\mathrm{T}}$, much more complicated behavior can occur [96]. Some of these low$p_{T}$ effects stem ultimately from saturation, which, from a string perspective, corresponds 
ATLAS Data at $\sqrt{\mathrm{s}}=8 \mathrm{TeV}$

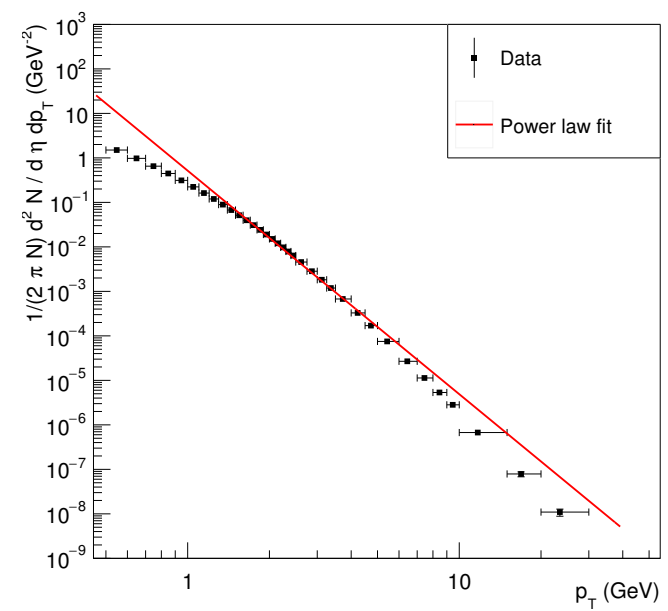

(a)

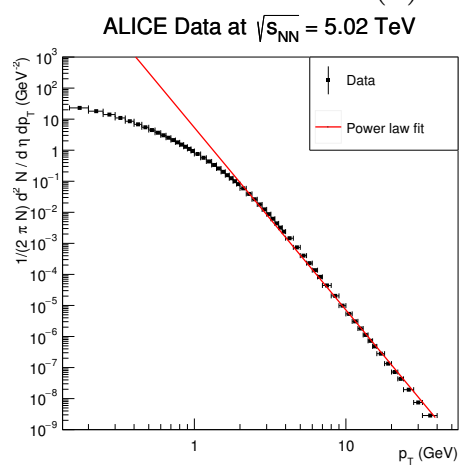

(c)

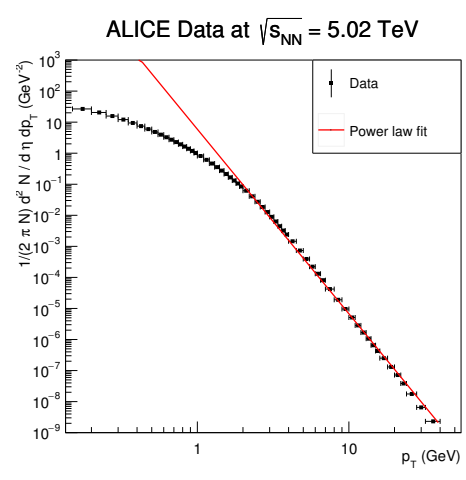

(d)
ATLAS Data at $\sqrt{\mathrm{s}}=13 \mathrm{TeV}$

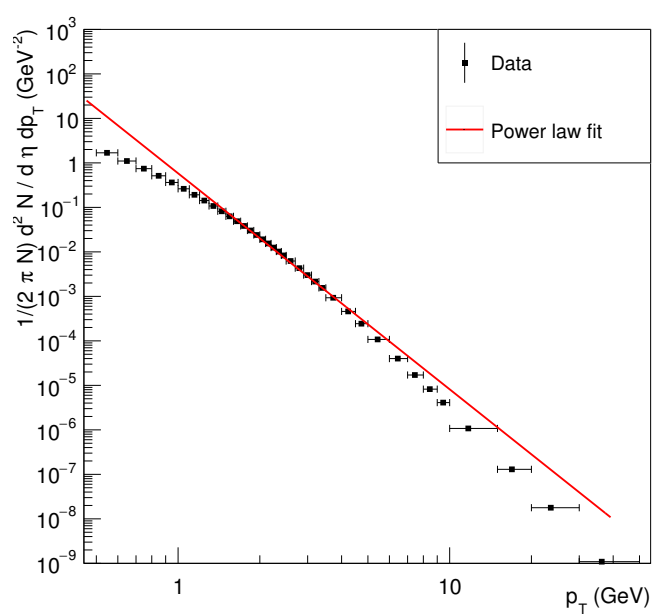

(b)

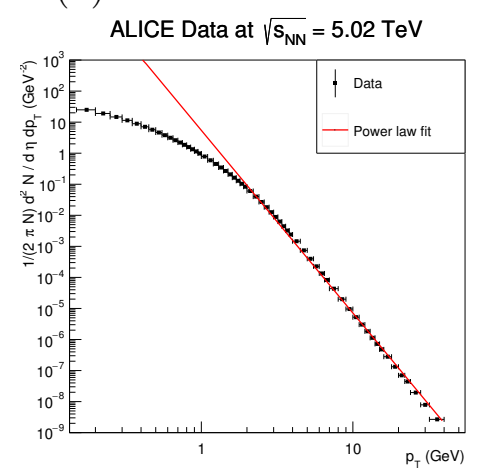

(e)

Figure 4. Pure power-law $\left(A / p_{T}^{B}\right)$ fits for the ATLAS $\sqrt{s}=8 \mathrm{TeV}$ (a) and $\sqrt{s}=13 \mathrm{TeV}$ (b) data sets, as well as the ALICE $\sqrt{s}=5.02 \mathrm{TeV}$ data set at rapidity bins of $|\eta|<0.3$ (c), $-1.3<\eta<-0.8$ (d), and $-0.8<\eta<-0.3$ (e) respectively.

to the emergence of eikonal physics in summing over string-loop diagrams. ${ }^{18}$ Other effects will be sensitive to confinement specifics which are partially avoided at large $p_{T}$ from the AdS/CFT perspective [30, 91]. This is borne out in the data by deviations from power-law behavior at small $p_{T}$ as can be seen in figure 4 . More details are given in appendix E.

To avoid these complications, we will attempt to allow for such behavior by including an offset $C$, expected to be of order $\Lambda_{\mathrm{QCD}}$, in our fit function. Thus, for production mediated by factorized Mueller diagrams, we want to fit a curve of the form

$$
\frac{1}{2 \pi p_{T}} \frac{d^{2} \sigma}{d p_{\mathrm{T}} d \eta}=\sum_{i} \frac{A_{i}}{\left(p_{T}+C\right)^{B_{i}}}
$$

where the $B_{i}$ are given by twice the conformal dimensions of the produced particles. More details about the reasoning leading to this fit function are given in appendix E.

\footnotetext{
${ }^{18}$ Eikonalization is also responsible for saturation in the context of DIS. More discussion will be provided at the end of this section.
} 


\begin{tabular}{|c|c|c|c|}
\hline Dataset & $\mathrm{A} / 10\left(\mathrm{GeV}^{-2}\right)$ & $\mathrm{B}$ & $\mathrm{C} /(1 \mathrm{GeV})$ \\
\hline ALICE 5.02 TeV,$|\eta|<0.3[95]$ & $38.48 \pm 8.26$ & $7.23 \pm 0.09$ & $1.32 \pm 0.04$ \\
\hline ALICE 5.02 TeV,$-0.8<\eta<-0.3[95]$ & $37.60 \pm 7.97$ & $7.22 \pm 0.08$ & $1.30 \pm 0.04$ \\
\hline ALICE 5.02 TeV,$-1.3<\eta<-0.8[95]$ & $43.00 \pm 9.29$ & $7.30 \pm 0.09$ & $1.31 \pm 0.04$ \\
\hline ATLAS $8 \mathrm{TeV}[94]$ & $4.46 \pm 2.60$ & $7.03 \pm 0.264$ & $1.07 \pm 0.123$ \\
\hline ATLAS 13 TeV [93] & $5.77 \pm 3.38$ & $6.96 \pm 0.265$ & $1.12 \pm 0.126$ \\
\hline
\end{tabular}

Table 1. Fitted values of parameters in eq. (5.2) for three data sets. Both central values and statistical errors are quoted.

Theoretically, our results are most strongly suited to describe glueballs. Because glueballs are not experimentally identifiable, we will instead focus on the production of other QCD bound states, namely mesons, via glueball decays. We will study meson production at the LHC in both proton-lead and proton-proton collisions. Within AdS/CFT, the dominant contribution should be from the production of scalar glueballs with $\Delta=4$ (and thus $B=8$ ) with double-Pomeron Mueller diagram, so for simplicity we will mostly focus on a fitting function given by

$$
\frac{1}{2 \pi p_{T}} \frac{d^{2} \sigma}{d p_{\mathrm{T}} d \eta}=\frac{A}{\left(p_{T}+C\right)^{B}}
$$

We will consider here three datasets. The first comes from proton-lead collisions studied by the ALICE collaboration at $\sqrt{s_{N N}}=5.02 \mathrm{TeV}$ [95], and the last two come from proton-proton collisions analyzed by the ATLAS Collaboration at center of mass energies of $\sqrt{s}=8[94]$ and 13 [93] TeV. These two categories are discussed in sections 5.1 and 5.2, respectively. Results of these studies are shown in table 1 . These results are interpreted in section 5.3.

By comparing analysis run on the various data sets we will be able to gain some insight into the (lack) of energy dependency in this kinematic regime. The ALICE datasets in particular have been run at various pseudorapidity, $\eta$, ranges which allows us to see that there is also essentially no variation in kinematics under changes in pseudorapidity. The ATLAS data has been collected at psuedorapidity range covered by the end caps $(|\eta|<2.7)[97]$, but this is still safely inside the central production limit.

\subsection{Proton-lead collisions and pseudorapidity dependence}

The data in [95] are binned in the pseudorapidity $\eta \cdot{ }^{19}$ There are three bins, corresponding to the $|\eta|<0.3,-0.8<\eta<-0.3$, and $-1.3<\eta<-0.8$ regimes, respectively. This allows us the opportunity to study the possible presence of a dependence on pseudorapidity at fixed $\sqrt{s}$. These data cover the range $0.15 \mathrm{GeV}<p_{\mathrm{T}}<50 \mathrm{GeV}$, and hence allow us to extend further into the high- $p_{\mathrm{T}}$ regime than the above analyses.

\footnotetext{
${ }^{19}$ To be precise, the binning in [95] is done not in terms of the usual pseudorapidity, but instead in terms of a shifted "center of mass" pseudorapidity. this technicality should not be important here, as it amounts to shifting the definitions of each bin by $\delta \eta=0.465$ [95].
} 


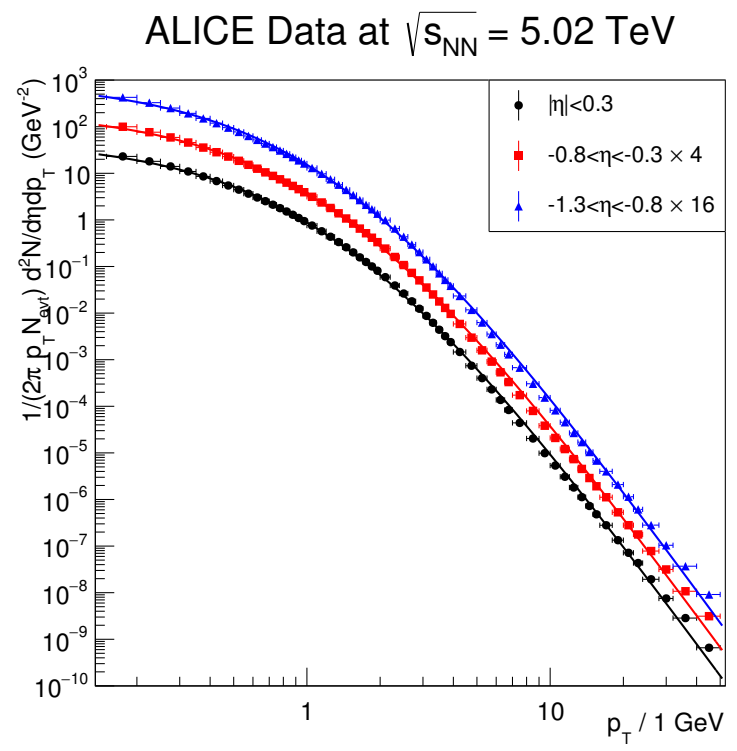

Figure 5. Fit of inclusive double-differential charged hadron production cross sections obtained in proton-lead collisions at center of mass energy $\sqrt{s}=5.02$ by the ALICE Collaboration, presented in [95]. Two of the datasets are rescaled by factors of four and sixteen for visual clarity. The data are displayed alongside fits to the model in eq. (5.2).

The results of the fits are shown in figure 5. Excellent agreement between the fit model and the data is seen in all three cases. This plot is visually suggestive that the kinematic dependencies depend very slightly, if at all, on the pseudorapidity bin; this is confirmed numerically by the results in table 1 . All three fit parameters are compatible in the three bins at the one sigma level.

\subsection{Proton-proton collisions and center of mass energy dependence}

ATLAS has also measured the inclusive double-differential single-hadron production cross section [93, 94]. Unlike the data discussed above, these data are presented in a single pseudorapidity bin, so we cannot extract any information about $\eta$ dependence. Instead, these two datasets allow us to study the validity of our model in the energy frontier; we have worked in the limit of large center of mass energy, so this is the regime where we expect our results to be the most directly applicable.

The results of the fit are shown in figure 6 . As before, the model is seen to correspond closely to data. Within one sigma, the results are seen to match between the two ATLAS datasets, although given the smaller number of data points the uncertainties are of course larger than in the above analysis. 


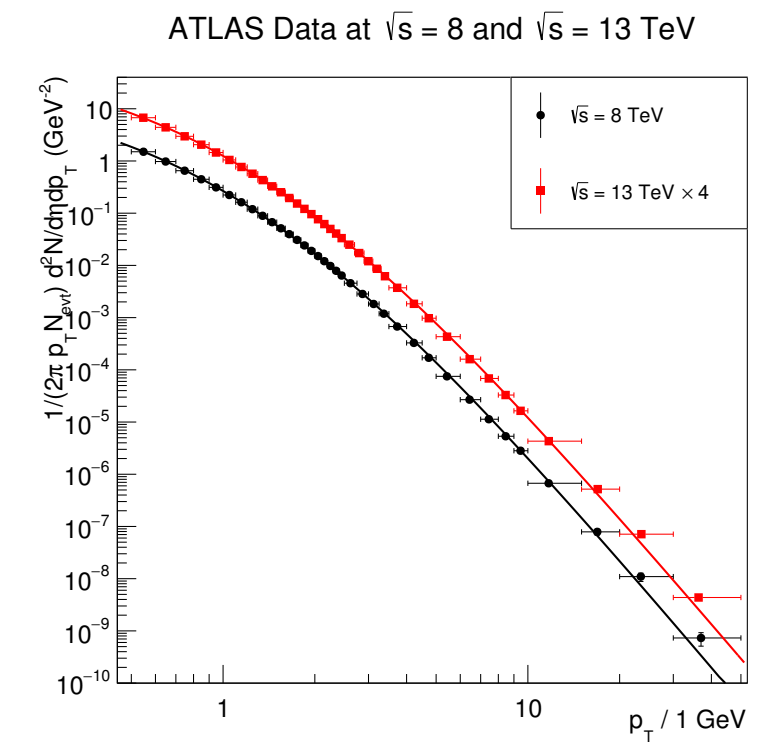

Figure 6. Fit of inclusive double-differential charged hadron production cross sections obtained in proton-proton collisions at center of mass energy $\sqrt{s}=8$ and $\sqrt{s}=13 \mathrm{TeV}$ by the ATLAS Collaboration, presented in [94] and [93], respectively. The $13 \mathrm{TeV}$ dataset is rescaled by a factor of four for visual clarity. The data are displayed alongside fits to the model in eq. (5.2).

\subsection{Interpretation}

Overall, the preceding results, summarized in table 1, match up rather well with our predictions. The fits are compatible at the two- $\sigma$ level with the power law exponent being independent of both the pseudorapidity and the center of mass measurement. This agrees with the results of section 4 . There are two important caveats, however. First, the overall normalization of the distributions varies sharply between the two types of measurements, with the proton-lead collisions seeming to have a cross section enhanced by an order of magnitude relative to the proton-proton collisons. That the overall normalizations vary so strongly is not altogether surprising. The holographic argument presented here does not offer an easy way to compute this prefactor, so we have no real prediction for it. Certainly we expect higherorder corrections, which are unaccounted for in our tree-level calculation, to importantly influence the normalization. Moreover, from considerations of the mechanisms for protonlead and proton-proton scattering, it is clear that the difference between these two can have a physical interpretation, rather than being interpreted as an artifact of our calculation.

Let us turn to our predicted value $B=8$ for scaling dimension. In [98], it was found that this value is consistent with low energy data. In a perturbative treatment for inclusive production, one generally expects a $p_{T}$-dependence of the type

$$
\frac{E d \sigma}{d p^{3}} \simeq F\left(p_{T} / \sqrt{s}\right) p_{T}^{-n}
$$

with $n=4$ for naive scaling. It is also interesting to point out that a picture based on "constituent-quark-interchange" [99] also leads to an effective value of $n \simeq 8$. However, our expectation of $n=8$ follows from the assumption that gluon dynamics dominates in 
central production and particle distribution follows that for production of scalar glueballs. It would be interesting to explore how the "constituent-quark-interchange" approach could be made compatible with our dual picture of strong-coupling AdS-Pomeron for central production in a gluon-dominated setting.

It is equally important to point out that the fitted values for the scaling dimension, although comparable, are not strictly compatible with the predicted value of $B=8$. In the context of our paper, that the experimental data do not appear strictly consistent with the interpretation of production mediated by $\Delta_{c}=4$ glueballs could be significant. In general, the best we can hope for from AdS/QCD is an understanding of event kinematics, so the value of the power law exponent is of central importance to our results. We therefore turn now to a discussion of this small but possibly significant discrepancy.

Deviations from conformality. The five values for the exponent $B$ are all consistent with $B \simeq 7$, which seems to correspond to a process with $\Delta \simeq 3.5$ instead of our expected $\Delta=4$. Given the small numerical uncertainties on our fits, it is extremely unlikely that this is a fluctuation, and we must reconcile this result with our expectations. We will outline below some possible explanations for this effect. Although we cannot conclusively claim that any or all of these suggestions completely explain the fit results, they are within the realm of possibility, and would provide conceptually appealing physical interpretations.

Note that, strictly speaking, our CFT prediction yields a power $B=2 \tau$, where $\tau$ is the twist, $\tau=\Delta-J, J$ being the spin. For scalar glueball, with $J=0$, we thus have $\tau=4$. A more appealing version has the additional power law terms originating in the production of object with twist $\tau \neq 4$. The dominant scaling behavior is due to the production of scalar glueballs, with $\tau=\Delta=4$. However, if there is a significant production via tensor glueballs, $\tau=4-2=2$, thus leading to a term with power $2 \tau=4$. If we allow production to be mediated by both types of glueballs, we would naturally find a cross section of the form

$$
\frac{1}{2 \pi p_{T}} \frac{d^{2} \sigma}{d p_{T} d \eta} \sim A\left(p_{T}+C\right)^{-B}+D\left(p_{T}+C\right)^{-E},
$$

where we expect $B \sim 8$ and $E \sim 4$. For $A \gg D$, at small $p_{T}$ the $\delta=4$ term will dominate (See appendix $\mathrm{E}$ for other small $p_{T}$ information) and at large $p_{T}$ the $\delta=2$ term dominates. In the crossover region of intermediate momentum, the two terms can compete, causing a lowering of the effective power law exponent to be lowered, as alluded to above.

Because of the competing effects of these two terms, it is difficult to fit a function of this form directly to data. However, it is possible to make some simplifying assumptions to demonstrate that it is at least a plausible model. If we expand the cross section in eq. (5.4) about intermediate momentum, fix by hand the values $B=8$ and $E=4$, and import the value of the offset $C$ from table 1, we can float the normalizations $A$ and $D$ to compare this model to data. Such a fit is shown in figure 7 for the ALICE data set with $-0.8 \leq \eta \leq-0.3$; to mitigate low- $p_{T}$ effects, we have discarded data with $p_{T}<3 \mathrm{GeV}$. We do not claim that this is a legitimate fit to data per se; instead we aim to show that such a two-term fit is not an unreasonable form for the cross section.

Along similar lines, one could imagine quark-antiquark $(q \bar{q})$ mixing becoming significant. The calculation in section 4 occurs at large $N_{c}$, where $q \bar{q}$ mixing is suppressed. 


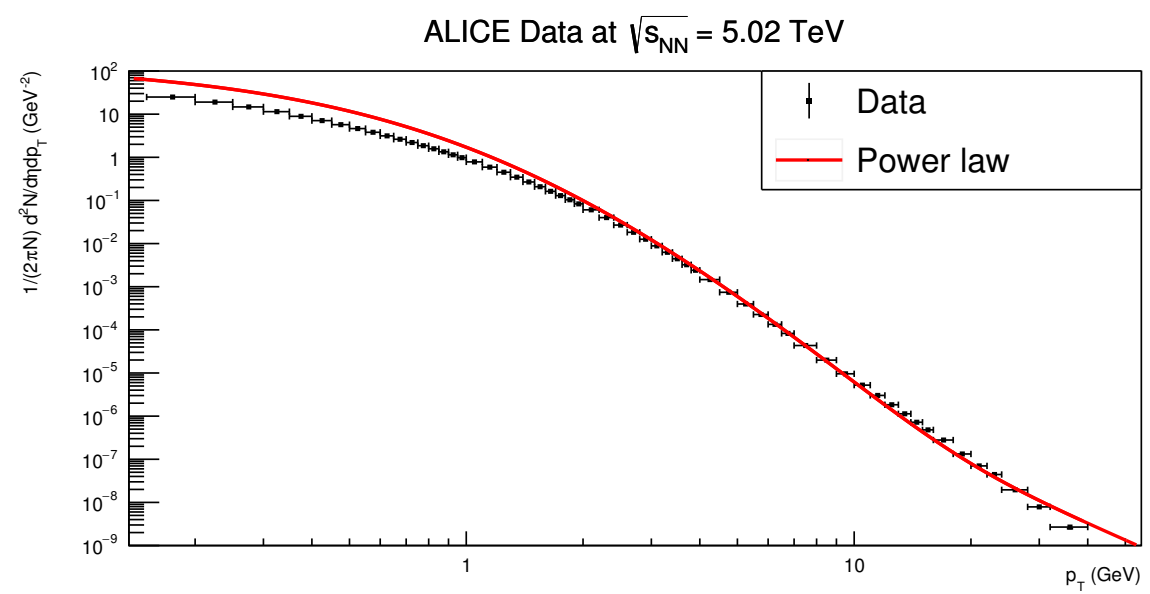

Figure 7. Two term power law fit to the first ALICE data set. A $3 \mathrm{GeV}$ cutoff has been used.

However, in real-world QCD we have $N_{c}=3$, so to obtain phenomenologically viable results it would be beneficial to consider the effects of glueballs mixing with $q \bar{q}$. One could imagine performing this calculation in a top-down Sakai-Sugimoto picture [100]. From a power-counting argument, we expect scalar $q \bar{q}$ states to lead to wavefunctions with $\tau_{c}=2$, and thus would contribute identically to a tensor glueball. It is unclear how these two scenarios might be distinguished either phenomenologically or experimentally.

As a last incarnation of this argument, we could have considered the effects of mixed Pomeron-Reggeon exchange; it was argued in $[24,25]$ that these contributions could remain important at LHC energies. Such Mueller diagrams could effect the $z$-cutoff in eq. (4.17), which would clearly have effected the final result. For instance, one might expect a correction of the order $\left(\sqrt{s} / p_{T}\right)^{-a} p_{T}^{-8} \sim s^{-1 / 2} p_{T}^{-8+2 a}$, with $a \simeq 1-2$, which will move the fit closer to the LHC data. In worldsheet terms, these diagrams would involve additional twist-two operators contributing to the $t$-channel OPEs. This could in general lead to an additional $\eta$-dependence of the final result, which a more refined treatment could become sensitive to.

As another possible line of reasoning, we can consider the effects of finite coupling. The earlier discussion mostly focused on the strong coupling limit of $\lambda \rightarrow \infty$. However, other attempts to fit holographic calculations to data have demonstrated that finite- $\lambda$ effects can be important $[27,29,30,91]$. In appendix B, we argue that the Reggeization of the graviton depends crucially on finite- $\lambda$ effects, i.e., from stringy physics beyond the supergravity limit. Thus, we expect finite- $\lambda$ physics to effect the glueball wavefunctions that must be convoluted with the scattering kernel. Related to this is the possibility of nontrivial anomalous dimensions. The central argument of this paper involved a holographic prediction for the kinematics of $\mathcal{N}=4$ SYM. In this theory, superconformality protects the conformal dimensions of scalar glueballs. However, real-world QCD has no such protection, and hence we might expect QCD glueballs to pick up nonvanishing anomalous dimensions. Such an effect could easily account for the observed deviation from $\Delta=4$ production.

Eikonalization. Another possibility for lowering the effective exponent is due to corrections coming from string-loops, although it is not immediately clear how such effect 
would emerge. See appendix B for more details. When the eikonal, eq. (B.11), becomes large, $\chi\left(s, \vec{b}, z, z^{\prime}\right)=O(1)$, multiple Pomeron exchange becomes important, leading to "saturation". Indeed, such effect should be important for inclusive production with $p_{T}=O\left(\Lambda_{Q C D}\right)$. Since this region depends crucially on how confinement deformation is implemented, our single-Pomeron analysis can be modified significantly. ${ }^{20}$ However, for production at large $p_{T}$, our current treatment should be reliable. Further study in this direction will be pursued.

Naive scaling. In a perturbative treatment for inclusive production, in the absence of dimensionful scales, the function $F$ in (5.3) would be dimensionless, leading to $n=4$; this is known as "naive scaling". However, our non-perturbative result in eq. (4.22), differs significantly from the naive scaling expectation, and the corresponding function $F$ in (5.3) depends also on confinement scale $\Lambda_{Q C D}$; this dependence enters through the "string cutoff" $z_{s}$ in eq. (4.20), as well as through the total cross ection $\sigma_{\text {total }}$. We note that LHC data has also been examined in $[102,105]$, against such naive expectation of $p_{T}^{-4}$. Clearly, this is not evident at LHC energies. This perturbative scaling law was also mentioned peripherally in [106]. Assuming the parameter $B$ is energy dependent, it was speculated in $[102,105]$ that one would reach $B \simeq 4$ at $s \sim 10^{3} \mathrm{TeV}$, far beyond the LHC range. Our study, on the other hand, is based on the belief that there are no unexpected new scales involved other than $\Lambda_{Q C D}$, and therefore that our AdS/CFT based analysis should be applicable at LHC energy.

\section{Summary and discussion}

We have explored the consequences of conformal invariance in inclusive QCD production at high energy by means of the AdS/CFT correspondence. As mentioned in section 1, although QCD is not strictly a CFT, it is nevertheless possible to address in certain kinematic limits where effects of confinement deformation are not expected to be important. In this treatment, we have focused on inclusive central production at large $p_{\perp}$ where we demonstrate that particle density obeys a power law fall-off that depends only on conformal dimension of the produced particle,

$$
\frac{1}{\sigma_{\text {total }}} \frac{d \sigma_{a b \rightarrow c+X}}{d^{3} \mathbf{p}_{c} / E_{c}} \propto \operatorname{Disc}_{M^{2}}\left\langle\mathcal{V}_{P} V_{c \bar{c}} \mathcal{V}_{P}\right\rangle \cdot \sim p_{\perp}^{-2 \tau_{c}}
$$

The analysis is carried out in a momentum-space setting. With inclusive cross sections as discontinuities, it is important to include stringy effects, e.g., taking the discontinuity for the matrix element of the central vertex, $V_{c \bar{c}}$, between two Pomeron vertex operators. As is the case of exclusive fixed-angle scattering, this power fall-off occurs due to the geometry of warped AdS space, via a generalized Polchinski-Strassler mechanism [13, 14]. The form of the power law is fixed by conformal invariance. This prediction appears to be well-supported by recent LHC data.

\footnotetext{
${ }^{20}$ For a perspective possibly different from ours, see [101]. A universal $e^{-c p_{T}}$ behavior for the region $p_{T}<\Lambda_{Q C D}$ was advocated in [102]. See also $[103,104]$ and appendix E. Since the data in this region is spare, a more conventional behavior such as $e^{-c^{\prime} p_{T}^{2}}$ cannot be ruled out.
} 
In the first part of this paper, we concentrated on more formal aspects of inclusive cross sections as discontinuities. We first focused on general CFT and useed DIS at small$x$ as an illustration on how to invoke a $t$-channel OPE. We next discussed AdS/CFT via Witten diagrams, and additionally introduced a confinement deformation in the IR. Lastly, we discuss gauge-string duality beyond the strict supergravity limit, which leads to the inclusion of stringy effects and, in turn, the AdS-Pomeron.

In the second part of the paper, we turned to the calculation of inclusive distribution for central production, with a particular focus on the kinematic limit of large $p_{\perp}$ production. We discussed the generalized optical theorem for $3 \rightarrow 3$ amplitude and computed the curved-space string theory prediction for the inclusive cross section, which lead to the conformal behavior in eq. (6.1). Finally, we test this finding by examining the recent LHC data, coming from both proton-lead and proton-proton collisions analyzed by the ALICE and ATLAS collaborations.

We end by mentioning some possible future directions for inclusive study of conformal invariance. On a more theoretical side, a better understanding on the $x$-space and $p$-space connection would be desirable. For a CFT with gravity dual, this can be done most easily through a perturbative Witten diagram approach. Another possible avenue of attack is through the use of Mellin representation, as discussed by Mack [107]. Equally interesting is to extend the study to multi-particle production [44, 47, 108, 109]. Other phenomenological applications include inclusive production in other kinematical regions where the consequences of conformality can appear, ${ }^{21}$ such as the triple-Regge limit, explore heavy quark production in the central region, ${ }^{22}$ and tetra quark production. ${ }^{23}$ Also interesting would be the study of two-point correlations, such as $\gamma^{*} \rightarrow c_{1}+c_{2}+X$ or $a+b \rightarrow X_{1}+c_{1}+c_{2}+X_{2}$. Study in some of these issues are currently underway.

\section{Acknowledgments}

The work of T.R. and C.-I T. are supported in part in part by the Department of Energy under contact DE-Sc0010010-Task-A. T.R. is also supported by the University of Kansas Foundation Professor grant. R.N. is funded by the Stanford University Physics Department, a Stanford University Enhancing Diversity in Graduate Eduction (EDGE) grant, and by NSF Fellowship number DGE-1656518.

\section{A Inclusive cross sections and applications}

Inclusive cross sections as discontinuities also follow from unitarity. Here we give more detail first on the single particle inclusive amplitude and also provide examples of the

\footnotetext{
${ }^{21}$ Single-particle inclusive cross section in other kinematic regions has been addressed in [98, 99, 106] from a perturbative dimensional counting perspective.

${ }^{22}$ For heavy quark production, this can be treated with the perturbative BFKL approach. See [110] and references therein.

${ }^{23}$ Although quark contributions are $1 / \mathrm{N}$ suppressed holographically- the $\Delta=4$ tetra quark contribution [111-113], which has already been investigated holographically [114], could compete with a scalar glueball.
} 

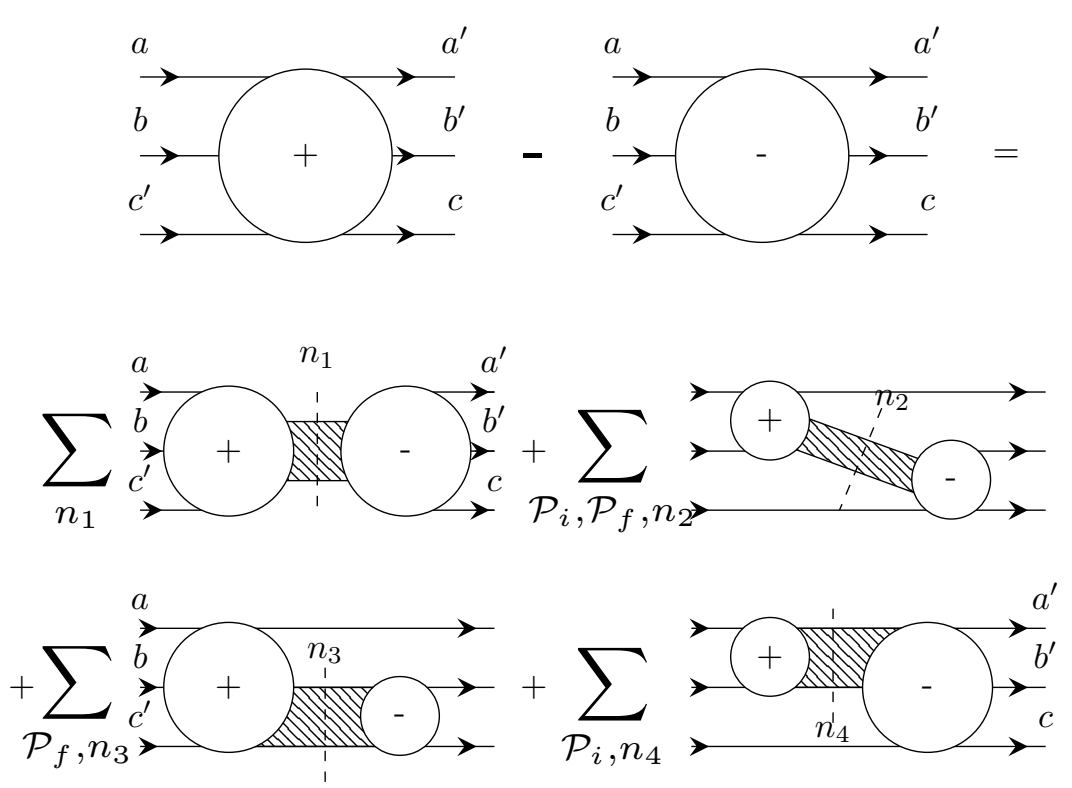

Figure 8. A schematic representation of unitarity equation for connected three-to-three scattering amplitudes from [116].

power of taking discontinuities to calculate cross sections. The issue of analytic structure is necessarily more involved in the case of CFT, which can be simplified in strong coupling via the use of Witten Diagrams in momentum-space representation.

\section{A.1 Single particle inclusive}

The discontinuity in eq. (2.7) is taken in the forward limit, where $p_{a^{\prime}}=p_{a}, p_{b^{\prime}}=p_{b}$, and $p_{c^{\prime}}=p_{c}$. This corresponds to a generalized optical theorem $[76,115,116]$, and is also known as the Mueller formula. Just as the familiar optical theorem in eq. (1.3) follows from unitarity for the 2-to-2 elastic amplitude, this $M^{2}$-discontinuity enters as a particular term in the 3-to-3 unitarity relation, schematically represented in figure 8 .

Each term on the right-hand-side of the equation can be identified as the discontinuity in an appropriate invariant [115]. There are four types of discontinuity diagrams, with $\mathcal{P}_{i}$ and $\mathcal{P}_{f}$ summing over all possible permutations of initial and final states, while the $n_{i}$-sum over all allowed states. The missing-mass discontinuity enters in the second group, i.e., that indicated by $n_{2}$ sum in the unitary equation.

The discontinuity in eq. (2.7),

$$
\frac{d \sigma_{a b \rightarrow c+X}}{d^{3} \mathbf{p}_{c} / E_{c}} \propto \frac{1}{2 i s} \operatorname{Disc}_{M^{2}>0} T\left(p_{a^{\prime}}, p_{b^{\prime}}, p_{c} ; p_{a}, p_{b}, p_{c^{\prime}}\right),
$$

is taken in the forward limit, where $p_{a^{\prime}}=p_{a}, p_{b^{\prime}}=p_{b}$, and $p_{c^{\prime}}=p_{c}$. This identification, as explained in section 2.1, is in exact correspondence to that for the free propagator. The discontinuity in $M^{2}$ enters as a term in the 3-to-3 unitarity relation, as represented schematically in figure 8 . In this figure, shaded bands represent allowed intermediate states and all amplitudes, indicated by circles, involved are connected. We denote amplitudes in the physical region by "+" and complex conjugation by "-". As also explained in 
section 2.1, each term on the right-hand-side of the unitarity equation can be identified as the discontinuity in an appropriate invariant [115].

From the perspective of the process $a+b+c^{\prime} \rightarrow a^{\prime}+b^{\prime}+c, M^{2}$ is a "cross-channel" invariant, as opposed to "direct-channel" invariants, such as $s_{a b}=\left(p_{a}+p_{b}\right)^{2}, s_{a b c}=$ $\left(p_{a}+p_{b}+p_{c^{\prime}}\right)^{2}$, etc. Because of the Steinman rule, there are no double-discontinuities in overlapping invariants in the physical region $[115,116]$. This discontinuity in $M^{2}$, eq. (2.7), yields a sum over all allowed multi-particle states $X$, multiplied by a delta function factor, $\delta\left(\left(p_{a}+p_{b}-q_{c}\right)^{2}-M_{X}^{2}\right)$. Each state $X$ contributes a term which is the product of an on-shell amplitude for $a+b \rightarrow c+X$ with its conjugate, $T_{a^{\prime} b^{\prime} \rightarrow c^{\prime} X} T_{a b \rightarrow c X}$. The total discontinuity involves a sum over each allowed state $X$; for each $X$, the sum involves an integral over the appropriate multi-particle phase space.

\section{A.2 DIS, OPE and anomalous dimensions}

As an explicit illustration, consider the inclusive scattering $\gamma^{*}+$ proton $\rightarrow X$ of a virtual photon with momentum $q$ off of a proton of momentum $p$ in the limit of $Q^{2}=-q^{2} \rightarrow \infty$ with $x=Q^{2} / s$ fixed. That is, one is dealing with the photon-proton total cross section, $\sigma_{\gamma^{*} p}^{\text {total }}$, as a function of $Q^{2}$ and $x$. This cross section can be expressed as a product of photon polarization vectors and the hadronic tensor, $W^{\mu \nu}(p, q)$, defined as the Fourier transform of the current commutator, $\left\langle p\left|\left[J^{\mu}(x), J^{\nu}(0)\right]\right| p\right\rangle$. It can be expressed in terms of two scalar structure functions,

$$
W^{\mu \nu}=F_{1}\left(x, Q^{2}\right)\left(g_{\mu \nu}-\frac{q_{\mu} q_{\nu}}{q^{2}}\right)+F_{2}\left(x, Q^{2}\right)\left(p_{\mu}+\frac{q_{\mu}}{2 x}\right)\left(p_{\nu}+\frac{q_{\nu}}{2 x}\right) .
$$

For virtual Compton scattering, $q+p \rightarrow q^{\prime}+p^{\prime}$, the amplitude $T^{\mu \nu}\left(p, q ; p^{\prime}, q^{\prime}\right)$ is given by the Fourier transform of the $\mathbf{T}$-product $\left\langle p^{\prime}\left|\mathbf{T}\left\{J^{\mu}(x) J^{\nu}(0)\right\}\right| p\right\rangle$. In the forward scattering limit, $p=p^{\prime}$ and $q=q^{\prime}, T^{\mu \nu}$ has a Lorentz covariant expansion similar to that of $W^{\mu \nu}$, with new form factors $\widetilde{F}_{\alpha}\left(x, Q^{2}\right)$ replacing $F_{\alpha}\left(x, Q^{2}\right)$. The hadronic tensor is related to the forward amplitude by the Optical Theorem, which implies that $W^{\mu \nu}(p, q)=\frac{1}{2 i} \operatorname{Disc}_{s>0} T^{\mu \nu}(p, q ; p, q)$. Treating the $F_{\alpha}\left(x, Q^{2}\right)$ as real-analytic functions of $x$ with a branch cut over $[0,1]$, one has ${ }^{24}$

$$
F_{\alpha}\left(x, Q^{2}\right)=2 \operatorname{Im} \widetilde{F}_{\alpha}\left(x, Q^{2}\right) .
$$

DIS in QCD is strictly speaking not conformal. However, it is possible to explore conformal dynamics if one assumes a fixed coupling and focuses on the kinematic region of small- $x$. DIS structure functions are strongly peaked phenomenologically at $x \rightarrow 0$, which can be used to infer the dominance of gluon dynamics, consistent with the large $N_{c}$ expectation $[26,27,30]$. This singular small-x behavior allows a direct measurement of the anomalous dimensions, $\gamma_{n}$, for twist-two operators, $\mathcal{O}_{n}$, since these operators dominate in the $t$-channel OPE of two currents, $J^{\mu}(x) J^{\nu}(0)=\sum_{n}|x|^{n} c_{n}^{\mu \nu} \mathcal{O}_{n}(0)$.

\footnotetext{
${ }^{24}$ As explained in [26], in a large- $N_{c}$ treatment for QCD, the discontinuity consists of an infinite sequence of delta-functions, coming from Regge recurrences for the proton. These discontinuities can also directly be related to $\sigma_{T}$ and $\sigma_{L}$ for transverse and longitudinal off-shell photons.
} 


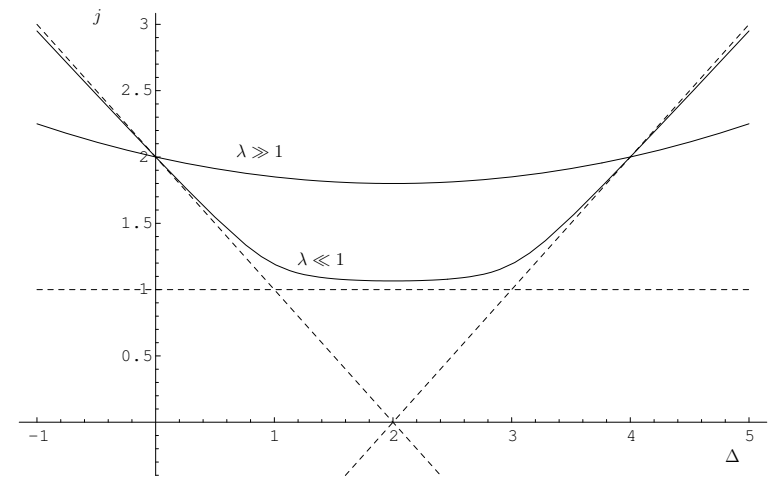

Figure 9. Schematic form of the $\Delta-j$ relation for twist-2 spectral curve at weak $(\lambda \ll 1)$ and strong coupling $(\lambda \gg 1)$, reproduced from ref. [15]. Symmetry about $\Delta=2$ follows from conformal invariance.

A standard analysis leads to an expansion for $\widetilde{F}_{\alpha}$ in $x^{-1}$, valid in the limit of large $Q^{2}$. Through a dispersion relation, the coefficients $M_{n}^{(\alpha)}\left(Q^{2}\right)$ of this expansion can be expressed as "moments" over its discontinuity across $0<|x|<1$, i.e., $M_{n}^{(\alpha)}\left(Q^{2}\right)=$ $\int d x x^{n-\alpha} F_{\alpha}\left(x, Q^{2}\right)$. In the large $Q^{2}$ limit, these coefficients are given approximately by

$$
M_{n}^{(\alpha)}\left(Q^{2}\right) \sim\left(Q^{2}\right)^{-\gamma_{n}} .
$$

Here, the $\gamma_{n}$ are the anomalous dimensions of twist-2 operators with even integer spin $j=n$, defined by

$$
\gamma(j)=\Delta(j)-j-2
$$

For $j=2$, we have $\gamma_{2}=0$ due to energy momentum conservation. ${ }^{25}$ For $j \neq 2$, anomalous dimensions do not vanish, which leads us directly to CFT dynamics. We will frequently treat $\Delta=j+2+\gamma(j)$ as a function of $j$, or, equivalently, its inverse, $j(\Delta)$, as a continuous function of $\Delta$, as shown in figure 9 . That is, by treating the structure functions as discontinuities, one can explore anomalous dimensions through a $t$-channel OPE, which can serve as a spring-board for introducing stringy effects via AdS/CFT. ${ }^{26}$ In particular, at large 't Hooft coupling $\lambda$, by exploring Regge behavior, one has $F_{s} \sim x^{-(2-s)-j_{0}}$ for $2>j_{0}>1$, where $\Delta\left(j_{0}\right)=2$ and is identified with the Pomeron intercept. In the strong coupling limit, $j_{0} \simeq 2-2 / \sqrt{\lambda}$. Clearly, exploring this holographically requires going beyond the SUGRA limit of $\lambda \rightarrow \infty$, which we turn to next.

\section{B AdS/CFT scattering and the BPST program}

We provide here further details of scattering in the AdS/CFT and give a brief summary of the BPST program [15-17], which constitutes the steps leading to eqs. (3.11) and (4.7).

\footnotetext{
${ }^{25}$ We are restricting ourselves here to the so-called single-trace conformal primaries. For other related discussion, see [117] and references therein. See section 5.3 and also appendix B for going beyond this restriction.

${ }^{26}$ Conformal invariance forces $j(\Delta)$ to be symmetric about $\Delta=2$, with $j(\Delta)$ having a minimum at $\Delta=2$. The intercept $j_{0}$ of the Pomeron [15], which obeys $2>j_{0}=1+\varepsilon>1$, can be found by demanding $\Delta\left(j_{0}\right)=2$. See also appendices B and C.
} 
In $[15,17]$, AdS/CFT is implemented by starting first with flat-space string theory. Alternatively, the construction of the BPST Pomeron can be initiated with a CFT OPE, and the corresponding Witten diagram expansion in the supergravity theory, and from there incorporate stringy effects $[20-23,51]$. The two approaches are equivalent, and provide separate intuitive frameworks. Here and in appendix $\mathrm{C}$ we will integrate both approaches.

$\boldsymbol{t}$-channel OPE and Witten diagrams. For 2-to-2 scattering at high energy, with $s=\left(p_{1}+p_{2}\right)^{2} \rightarrow \infty$ and $t=\left(p_{3}-p_{1}\right)^{2}<0$, the simplest Witten diagram that appears in the $t$-channel OPE is that from a single scalar exchange. In a momentum-space representation, up to a constant it is given by

$$
T_{\text {dilaton }}\left(s, t, p_{i}^{2}\right)=\int d \mu(z) d \mu\left(z^{\prime}\right) \Phi_{1}\left(z, p_{1}^{2}\right) \Phi_{3}\left(z, p_{3}^{2}\right) G_{F}\left(z, z^{\prime}, t\right) \Phi_{2}\left(z^{\prime}, p_{2}^{2}\right) \Phi_{4}\left(z^{\prime}, p_{4}^{2}\right),
$$

where $d \mu(z)=d z \sqrt{-g}$ is the $A d S_{5}$ measure and $G_{F}\left(z, z^{\prime}, t\right)$ is the scalar bulk-to-bilk propagator given in eq. (3.5). In anticipation of the confinement deformation we will later introduce, we will replace bulk-to-boundary propagators $\Phi_{i}\left(z, p^{2}\right)$ with normalizable physical wave functions $\varphi_{i}(z)$ in what follows.

In a Minkowski setting, the exchange of a spin $J$ excitation leads to a contribution whose growth is bounded from above by $s^{J}$. Therefore, the $t$-channel scalar exchange in eq. (B.1) is independent of $s$. For $\mathcal{N}=4 \mathrm{SYM}$, in the extreme limit $\lambda=g^{2} N_{c} \rightarrow \infty$ of large 't Hooft coupling, the dominant Witten diagram comes from the exchange of one $J=2$ graviton. This diagram has a form similar to that in eq. (B.1) but with the scalar bulk-to-bulk propagator replaced with a tensor propagator $G_{M N M^{\prime} N^{\prime}}\left(z, z^{\prime}, t\right)$, and the factors of the coupling at the vertices replaced by the conserved energy-momentum tensors $T^{M N}$ and $T^{M^{\prime} N^{\prime}}$. At large $s$ and fixed $t$, the dominant contribution comes from the $(++--)$ helicity component, which couples to the large light cone momenta $\left(z z^{\prime}\right)^{4}\left(p_{1}^{+}\right)^{2}\left(p_{2}^{-}\right)^{2}$. Thus, in this limit we have

$$
T_{\text {graviton }}\left(s, t, p_{i}^{2}\right) \simeq \int d \mu(z) d \mu\left(z^{\prime}\right) \Phi_{13}(z) \widetilde{\mathcal{K}}_{G}\left(s, t, z, z^{\prime}\right) \Phi_{24}\left(z^{\prime}\right),
$$

where the graviton kernel can be expressed in terms of scalar propagator $G_{F}\left(z, z^{\prime}, t\right)$ and the red-shifted energy invariant $\widetilde{s}$ as $\widetilde{\mathcal{K}}_{G}=G_{++--} \widetilde{s}^{2}=\left(z z^{\prime}\right)^{-2} G_{F}\left(z, z^{\prime}, t\right) \widetilde{s}^{2}$. We have also defined vertex factors $\Phi_{13}(z)=z^{2} \varphi_{1}\left(z, p_{1}^{2}\right) \varphi_{3}\left(z, p_{3}^{2}\right)$ and $\Phi_{24}\left(z^{\prime}\right)=z^{\prime 2} \varphi_{2}\left(z^{\prime}, p_{2}^{2}\right) \varphi_{4}\left(z^{\prime}, p_{4}^{2}\right)$. We therefore see that the amplitude scales as $s^{2}$, as expected. Schematically, we write this as

$$
T_{\text {graviton }}(s, t)=\Phi_{13} * \widetilde{\mathcal{K}}_{G} * \Phi_{24} .
$$

where $*$ corresponds to integration over the AdS bulk.

Ultralocal scattering and the Polchinski-Strassler mechanism. It has been stressed in [13] that scattering amplitudes in gauge theories with a good string dual description can often be simplified, since the dual ten-dimensional string scattering on $A d S_{5} \times S^{5}$ is effectively local. This simplification is particularly applicable in the limit of fixed anglescattering when all four-dimensional Mandelstam invariants are large and of the same order. In this limit, gauge theory amplitudes can be expressed as a coherent sum of local 
scattering in the AdS bulk, where again we ignore fluctuations in $S^{5}$ throughout [13]. As an effective five-dimensional scattering process, the momenta $p^{\mu}$ for external states are seen by local observers in the AdS bulk to be red-shifted, with large components $\widetilde{p}^{\mu}$ along $p_{i}^{\mu}$ where

$$
\widetilde{p}_{i}^{\mu} \simeq(z / R) p_{i}^{\mu} .
$$

We are interested in a strongly coupled boundary theory, so as above we take the AdS radius $R$ large compared to the string scale. In what follows, we shall set $R=1$.

In this limit, a 4-D scattering amplitude reduces to a coherent sum over local scattering in the AdS bulk, so that

$$
T_{n}\left(p_{1}, p_{2}, \cdots\right)=\int \frac{d z}{z} \mathcal{T}_{n}\left(\tilde{p}_{1}, \tilde{p}_{2}, \cdots\right) \Pi_{i}\left\{\varphi_{i}(z)\right\},
$$

where $\mathcal{T}_{n}$ corresponds to the amputated bosonic string Green's function in flat space. In terms of invariants, the arguments for $\mathcal{T}_{n}$ are red-shifted, $s_{i j} \rightarrow z^{2} s_{i j}$. A flat-space bosonic 4-point amplitude can be expressed in a Koba-Nelson representation involving an integral over a single modulus. In the limit of $-t \simeq\left(1-\cos \theta_{\mathrm{cm}}\right) s \rightarrow \infty$, the integral is dominated by a saddle point. This leads to an exponential cutoff,

$$
\mathcal{A}_{4}(s, t) \sim e^{-f\left(\cos \theta_{c m}\right) \alpha^{\prime} s} .
$$

More details are provided in appendix D. This exponential suppression is a generic feature of flat-space string scattering, and also holds for multi-particle scattering in similar generalized fixed-angle limits. ${ }^{27}$ As stressed in [13], the exponential suppression in eq. (B.6) allows us to restrict the domain of integration in eq. (B.5) to an effective scattering region $z \in$ $\left[0, z_{s}(s)\right]$, where $z_{s}(s)=O(1 / \sqrt{s})$. We will refer to this simplification as the PolchinskiStrassler mechanism. In the scattering region, $\mathcal{T}_{4}(\widetilde{s}, \widetilde{t})=O(1)$. This, combined with the wavefunctions in eq. (3.9), leads to a power-law falloff for the cross section of the form $\frac{d \sigma}{d t} \sim s^{-\tau_{\text {total }}}$, where $\tau_{\text {total }}$ is the sum of the twists $\tau_{i}=\Delta_{i}-J_{i}$ of the external particles. This is consistent with the dimensional counting rule of [73-75].

Beyond the SUGRA limit. The standard Witten expansion involves only propagators and vertices of super-gravity fields in $A d S_{5}$, such as the dilaton $\varphi$, metric fluctuations $h_{\mu \nu}$, and the anti-symmetric tensor $B_{\mu \nu}$. This dramatic reduction in the number of degrees of freedom can be understood in terms of the boundary theory by the rapid increase of anomalous dimensions for all unprotected gauge-invariant local operators in the large 't Hooft coupling limit. Generically, their conformal dimensions grow as

$$
\Delta(j)=j+2+\gamma_{j}=O(\sqrt{\lambda}),
$$

so that in the $\lambda \rightarrow \infty$ limit their string duals become heavy and decouple. In this limit of the sum can often be truncated so that it is given approximately by sums of perturbative $t$-, $s$ - and $u$-channel exchange diagrams. Perturbatively each of these diagrams will contribute only to discontinuities in their respective channel.

\footnotetext{
${ }^{27}$ Indeed, as mentioned in section 1 , this feature represents a serious failure for earlier attempt in formulating gauge theories as strings.
} 
From the graviton to the BPST pomeron. In a $t$-channel OPE, the contribution from a conformal primary with definite spin does not lead to singularities in the cross channel invariants $s$ and $u$. Discontinuities can emerge due to re-summation of high-spin exchanges. For finite 't Hooft coupling, incorporating the higher string modes associated with the graviton leads to a "reggeized AdS graviton". This in turn leads to the BPST program, where elastic amplitudes at high energy can be represented schematically in a factorizable form like that of 2-to-2 amplitude in eq. (3.11).

Here the universal Pomeron kernel $\widetilde{\mathcal{K}}_{P}$ grows with a characteristic power behavior at large $s \gg|t|$, i.e. $\widetilde{\mathcal{K}}_{P} \sim s^{j_{0}}$. The strong-coupling Pomeron intercept, at leading order in $\lambda$, is $j_{0}=2-2 / \sqrt{\lambda}$, which agrees with spin $J=2$ of the graviton in the limit of $\lambda=g^{2} N_{c} \rightarrow \infty$. Conversely, at finite $\lambda$, the regggeized AdS graviton has its intercept lowered below $J=2$. More generally, this approach leads to conformal Regge theory in CFT, which we will discuss briefly in section C. Holographic descriptions of scattering data agree with a Pomeron intercept near $j_{0} \simeq 1.3$ in a strongly coupled regime [27, 77].

At finite $\lambda$, one can incorporate higher string modes through a Pomeron vertex operator via a world-sheet OPE. More directly, one can adopt a $J$-plane formalism, where the Pomeron kernel $\widetilde{\mathcal{K}}_{P}$ can be given by an inverse Mellin transform, as in eq. (3.12),

$$
\widetilde{\mathcal{K}}_{P}\left(s, t, z, z^{\prime}\right)=-\int_{L-i \infty}^{L+i \infty} \frac{d j}{2 \pi i}\left(\alpha^{\prime} \widetilde{s}\right)^{j} \frac{1+e^{-i \pi j}}{\sin \pi j} \widetilde{G}_{j}\left(t, z, z^{\prime}\right),
$$

with $\operatorname{Re}\left(j-j_{0}\right)=L>0$. Due to curvature of AdS, the effective spin of a graviton exchange is lowered from 2 to $j_{0}<2$. The propagator $\widetilde{G}_{j}\left(z, z^{\prime} ; t\right)$ can be found via a spectral analysis in either $t$ or $j$. Let us focus on the conformal limit. Holding $j>j_{0}$ real and working at leading order in $\lambda$, the spectrum in $t$ can be seen to be continuous along its positive real axis leading to

$$
\widetilde{G}_{j}\left(z, z^{\prime} ; t\right)=\int_{0}^{\infty} k d k \frac{J_{(\Delta(j)-2)}(k z) J_{(\Delta(j)-2)}\left(k z^{\prime}\right)}{k^{2}-t},
$$

where $\Delta(j)=2+\sqrt{2 \sqrt{\lambda}\left(j-j_{0}\right)}$. At $J=2$, this reduces to the graviton kernel.

An alternative spectral representation in $j$ has also been provided in [15]. The leading contribution to eq. (3.12) comes from a branch-cut at $j_{0}$ which corresponds to a coherent sum of contributions from string modes associated with the graviton. At $s$ large and $t=0$ the kernel becomes

$$
\widetilde{\mathcal{K}}_{P}\left(s, 0, z, z^{\prime}\right)=-\left(\frac{1+e^{-i \pi j_{0}}}{\sin \pi j_{0}}\right)\left(\alpha^{\prime} \widetilde{s}\right)^{j_{0}},
$$

up to log corrections. This is the form we adopt for inclusive central production.

Tensor glueballs and confinement deformation. Consider next the addition of a confinement deformation, leading to a theory with a discrete hadron spectrum, e.g. tensor glueballs lying on the Pomeron trajectory. To gain a qualitative understanding, it is instructive to rely on the "hard-wall" model, where the AdS coordinate $z$ is restricted to lie in the range $\left[0, z_{\max }\right]$; we take $z_{\max } \sim 1 / \Lambda_{Q C D}$. This model captures key features of confining theories with string theoretic dual descriptions. The propagator is now given by 
a discrete sum over allowed states as

$$
\widetilde{G}_{j}\left(z, z^{\prime} ; t\right)=\left(z z^{\prime}\right)^{-2} \sum_{n} \frac{\widetilde{\varphi}_{n}(z, j) \widetilde{\varphi}_{n}\left(z^{\prime}, j\right)}{m_{n}^{2}(j)-t}
$$

where $\widetilde{\varphi}_{n}(z, j)$ can be expressed in terms of Bessel functions. Eq. (B.10) extends eq. (3.7) to a sum over Regge trajectories.

Eikonalization. From a string-dual perspective, summing higher order string diagrams leads to an eikonal summation. More generally, eikonalization assures s-channel unitarity. Near-forward scattering in the high energy limit is referred to in some literature simply as the eikonal limit; this limit corresponds to $s \rightarrow \infty$ and $t$ fixed, leading to a CM frame scattering angle $\theta$ that vanishes as $\theta \sim 1 / \sqrt{s}$. Under plausible assumptions, it can be shown that flat-space scattering in this limit is determined by the integration of an eikonal phase, $\chi(s, \vec{b})$, over the two-dimensional space of impact parameters $\vec{b}$. In this eikonal form the reduced 5 -D momentum transfer squared serves as a 3 -d Laplacian, $\widetilde{t} \rightarrow \nabla_{A d S_{\perp}}^{2}$; and there is a diffusion kernel in 3-dimensional transverse space, between $\left(x_{\perp}, z\right)$ and $\left(x_{\perp}^{\prime}, z^{\prime}\right)$. In the eikonal limit [16-23], one finds

$$
T_{1+2 \rightarrow 3+4}(s, t) \simeq(-2 i s) \int d \vec{b} e^{-i \vec{q}_{\perp} \cdot \vec{b}} \int d z d z^{\prime} \Psi_{(13)}(z) \Psi_{(24)}\left(z^{\prime}\right)\left\{e^{i \chi\left(s, \vec{b}, z, z^{\prime}\right)}-1\right\},
$$

where $\vec{b}=x_{\perp}^{\prime}-x_{\perp}$ is the impact parameter. Expanding to first order, we thus can identify our Pomeron kernel with the eikonal $\chi\left(s, \vec{b}, z, z^{\prime}\right)$ as

$$
\widetilde{\mathcal{K}}_{P}\left(s, t, z, z^{\prime}\right)=2 s \int d \vec{b} e^{-i \vec{q}_{\perp} \cdot \vec{b}} \chi\left(s, \vec{b}, z, z^{\prime}\right)
$$

When the eikonal becomes large, $\chi\left(s, \vec{b}, z, z^{\prime}\right)=O(1)$, multiple Pomeron exchange becomes important leading to effects like saturation. For many purposes, for example DIS at HERA, keeping a single Pomeron contribution is often sufficient. For p-p and p- $\mathrm{Pb}$ scatterings, eikonalization is also phenomenologically important. This can be seen in effects like "taming" the power increase for total cross sections with $s^{\varepsilon}$ to $\log ^{2} s$, etc.

\section{Conformal partial-wave and Regge theory}

The Regge limit for CFT can also be addressed more directly by analytically continuing the Euclidean OPE to Minkowski space. We will now briefly discuss this approach, which will lead us to an alternate derivation of eq. (3.12). We will focus on a four-point correlation function of primary operators $\mathcal{O}_{i}$ of dimensions $\Delta_{i}$. For a $t$-channel OPE, it is customary to express the 4-point correlation function for external scalars as

$$
\left\langle 0\left|\mathcal{O}_{1}\left(x_{1}\right) \mathcal{O}_{3}\left(x_{3}\right) \mathcal{O}_{2}\left(x_{2}\right) \mathcal{O}_{4}\left(x_{4}\right)\right| 0\right\rangle=\frac{1}{\left(x_{13}^{2}\right)^{\Delta_{1}}\left(x_{24}^{2}\right)^{\Delta_{2}}} F(u, v),
$$

where we define $x_{i j}=x_{i}-x_{j}$ and the invariant cross ratios $u=\frac{x_{13}^{2} x_{24}^{2}}{x_{12}^{2} x_{34}^{2}}$ and $v=\frac{x_{14}^{2} x_{23}^{2}}{x_{12}^{2} x_{34}^{2}}$. For simplicity we have assumed $\Delta_{1}=\Delta_{3}$ and $\Delta_{2}=\Delta_{4}$. To explore conformal invariance, 
one normally begins with a conformal partial wave expansion [20-23], starting first in an Euclidean setting, where the connected component of the amplitude $F(u, v)$ is given by a sum over conformal blocks,

$$
F(u, v)=\sum_{j} \sum_{\alpha} C_{\alpha, j}^{(13),(24)} G\left(j, \Delta_{\alpha}(j) ; u, v\right) .
$$

For planar $\mathcal{N}=4 \mathrm{SYM}$, we restrict the sum to single-trace conformal primary operators.

\section{C.1 OPE in Minkowski setting}

The conformal Regge limit corresponds to a double light-cone limit in a Minkowski setting. This light-cone limit for the OPE corresponds to $u \rightarrow 0$ and $v \rightarrow 1$, with $(1-v) / \sqrt{u}$ fixed. Equivalently, by introducing $u=z \bar{z}$ and $v=(1-z)(1-\bar{z})$ with $z=\sigma e^{\rho}$ and $\bar{z}=\sigma e^{-\rho}$, the precise Regge limit can also be specified by

$$
\sigma \rightarrow 0, \quad \rho \text { fixed. }
$$

In a frame where $x_{1 \perp}=x_{3 \perp}$ and $x_{2 \perp}=x_{4 \perp}$, this limit corresponds to approaching the respective null infinity while keeping the relative impact parameter $b_{\perp}=x_{1 \perp}-x_{2 \perp}$ fixed.

To make contact with Regge theory, it is useful to adopt a more general starting point. We introduce a basis $\mathcal{G}(j, \nu ; u, v)$ of functions for the principle unitary conformal representation of the four-dimensional conformal group $\mathrm{SO}(5,1)$ and then expand $F(u, v)$ in terms of this basis as

$$
F(u, v)=\sum_{j} \int_{-\infty}^{\infty} \frac{d \nu}{2 \pi} a(j, \nu) \mathcal{G}(j, \nu ; u, v) .
$$

The conformal harmonics $\mathcal{G}(j, \nu ; u, v)$ are eigenfunctions of the quadratic Casimir operator of $\mathrm{SO}(5,1)$. Eq. (C.3) combines a discrete sum in the spin $j$ and a Mellin transform in a complex $\Delta$-plane, with $\Delta=2+i \nu$. To recover the standard conformal block expansion, one can close the contour in the $\nu$-plane [107], picking up dynamical poles in $a(j, \nu)$, at $\nu(j)=-i(\Delta(j)-2)$, thus arriving at eq. (C.1). These dynamical poles correspond to the allowed conformal primaries $\mathcal{O}_{\Delta(j)}$ of spin $j$ and dimension $\Delta(j)$.

In continuing to the Minkowski limit, it is necessary to work with conformal harmonics $\widetilde{\mathcal{G}}(j, \nu ; u, v)$ which are eigenfunctions of $\mathrm{SO}(4,2)$ Casimir with two continuous indices, $\nu$ and $j$. A distinguishing feature for the Minkowski conformal harmonics is the fact that, in the Regge limit, $\widetilde{\mathcal{G}}(j, \nu ; u, v) \sim \sigma^{1-j} \Omega_{i \nu}(\rho)=\sigma^{1-j} \frac{1}{4 \pi^{2}} \frac{\nu \sin (\nu \rho)}{\sinh \rho}$, so that the $\widetilde{\mathcal{G}}(j, \nu ; u, v)$ are more and more divergent for increasing $j>1$ as $\sigma \rightarrow 0$. It follows that the conventional discrete sum over spin would no longer converge. As explained in [51], a Sommerfeld-Watson resummation leads to a double-Mellin representation

$$
F(u, v)=-\int_{L-i \infty}^{L+i \infty} \frac{d j}{2 \pi i} \sum_{\tau= \pm} \frac{1+\tau e^{-i \pi j}}{\sin \pi j} \int_{-\infty}^{\infty} \frac{d \nu}{2 \pi} a_{\tau}(j, \nu) \widetilde{\mathcal{G}}(j, \nu ; u, v)
$$

where the contour in $j$ is to stay to the right of singularities of $a_{\tau}(j, \nu)$. The factor, $\frac{1+\tau e^{-i \pi j}}{\sin \pi j}$, is referred to as the "signature factor". We will in what follows consider even signature case, $\tau=+$. For more discussions, see [51]. 


\section{C.2 Conformal Regge theory and eikonal}

Conformal Regge theory assumes that $a(j, \nu)$ is meromorphic in the $\nu^{2}-j$ plane, with poles specified by the collection of allowed spectral curves $\Delta_{\alpha}(j)$. An example of such an $a$ is $a(j, \nu)=\sum_{\alpha} \frac{r_{\alpha}(j)}{\nu^{2}+\left(\Delta_{\alpha}(j)-2\right)^{2}}$. In the Regge limit, for even signature, $\tau=+$, the spectral curve associated with the energy-momentum tensor plays the dominant role. Here $\Delta_{P}(2)=4$ and this spectral curve is where the Pomeron singularity lies, as in figure 9. Keeping this contribution only leads directly to the Pomeron kernel in eq. (3.12). For more discussions, see [27, 29] and [15, 51].

In flat-space, by expanding eq. (B.11) to first order in $\chi$ and applying eq. (1.3), one can see that exchanging the eikonal once contributes to the total cross section, so that $\sigma_{\text {total }}(s) \simeq 2 \int d \vec{b} \chi_{I}(s, \vec{b})+O\left(\chi^{2}\right)$, where $\chi_{I}>0$ is the imaginary part of the eikonal. With AdS/CFT, it is possible to associate the eikonal with the leading $t$-channel exchange, as is done in [15]. The result is the leading (Pomeron) kernel, given by eq. (B.12), and repeated here,

$$
\widetilde{\mathcal{K}}_{P}\left(\widetilde{s}, \widetilde{t}, z, z^{\prime}\right)=\int d \vec{b} e^{i \vec{q}_{\perp} \cdot \vec{b}} \mathcal{K}_{P}\left(s, \vec{b}, z, z^{\prime}\right) \simeq(2 s) \int d \vec{b} e^{i \vec{q}_{\perp} \cdot \vec{b}} \chi\left(s, \vec{b}, z, z^{\prime}\right),
$$

where $\widetilde{s}$ and $\widetilde{t}$ are redshifted holographic invariants, as in eq. (B.4). In the conformal limit, eq. (C.5) provides a representation for a general scattering kernel. The eikonal $\chi$ encodes all dynamical information and, due to conformal symmetry, depends only on $\widetilde{s}=z z^{\prime} s$ and $\cosh \xi=\frac{z^{2}+z^{\prime 2}+b_{\perp}^{2}}{2 z z^{\prime}}$, where $\cosh \xi$ corresponds to a transverse chordal distance. The Regge limit is now $\widetilde{s} \rightarrow \infty$ with fixed $\xi$. It is important to note that the conformal representation (C.5) is valid for any value of the coupling constant, since it relies only on conformal invariance. We end by providing a Regge Dictionary for CFT:

$$
F(u, v) \leftrightarrow \chi(\widetilde{s}, \xi) ; \quad \sigma=\sqrt{u} \leftrightarrow \widetilde{s}^{-1} ; \quad \cosh \rho \approx \frac{1-v}{2 \sqrt{u}} \leftrightarrow \cosh \xi=\frac{b_{\perp}^{2}+z^{2}+z^{\prime 2}}{2 z z^{\prime}} .
$$

For more details, see [51].

\section{Flat-space string amplitudes}

Here we describe and evaluate some flat-space string amplitudes. As an illustration, we will begin with tree-level amplitudes for tachyons in bosonic string theory. The four-point open-string tachyon amplitude, known as the Veneziano amplitude, can be expressed in a Koba-Nielson form as

$$
\mathcal{A}_{0}(s, t)=\int_{0}^{1} d w(1-w)^{-2-\alpha^{\prime} s} w^{-2-\alpha^{\prime} t} .
$$

This is a planar-ordered amplitude, with singularities in $s$ and $t$ only. The full amplitude is given as a sum of three planar amplitudes, with singularities in $(t, u)$ and $(u, s)$ respectively, $\mathcal{A}_{\text {open }}(s, t)=\mathcal{A}_{0}(s, t)+\mathcal{A}_{0}(t, u)+\mathcal{A}_{0}(u, s)$. Since the external particles are tachyons, we have $\alpha^{\prime}(s+t+u)=-4$. The corresponding 4-point closed-string tachyon amplitude is the Virasoro amplitude, given by

$$
\mathcal{A}_{\text {closed }}(s, t)=\int d^{2} w|w|^{-4-\alpha^{\prime} t / 2}|1-w|^{-4-\alpha^{\prime} s / 2},
$$


where for closed strings $\alpha^{\prime}(s+t+u)=-16$. Unlike the Veneziano amplitude, the Virasoro amplitude contains singularities in all three channels. There exists closed-form expressions for these integrals in terms of $\Gamma$-functions.

Fixed-angle limit for 4-point amplitudes. For four-point scattering, the limit of fixed angle scattering is given by large $s$ and $t$, with $s / t$ held fixed. In the CM frame, we then have

$$
t \simeq-s(1-\cos \theta) / 2
$$

It is possible to read off the behavior for the Veneziano formula directly, but it is more instructive to work with the Koba-Nielson representation. Consider the open-string amplitude. When $s$ and $t$ are both large, with $t / s$ fixed, the integrand has a saddle point at $w^{*}=t /(s+t)$. When the integral is appropriately defined by analytic continuation, this saddle-point indeed dominates [118], and we then have

$$
\mathcal{A}_{0}(s, t) \sim e^{-f(\theta)\left(\alpha^{\prime} s\right)} .
$$

A similar analysis applies to all three terms for $\mathcal{A}_{\text {open }}(s, t)$. This property is clearly also shared for closed string amplitudes. It can be shown that the integral for $\mathcal{A}_{\text {closed }}(s, t)$ is again dominated by the saddle-point at $w^{*}=t /(s+t)$, thus leading to an expression like that in eq. (D.4) but with $\alpha^{\prime}$ replaced by $\alpha^{\prime} / 2$. This represents a generic property, which also applies to multiparticle amplitudes: in the fixed-angle limit where all invariants are large with relative ratios fixed, all flat-space string amplitudes are exponentially suppressed.

Regge limit for 4-point amplitudes. In the Regge limit of $s \rightarrow \infty$ with $t$ fixed, the saddle-point $w^{*}$ moves to one of the end-points of the domain of integration, $w=0$, and the amplitude can no longer be evaluated at $w^{*}$. Instead, we must sum the contributions from $w=O(1 / s)$. In [27], it was shown that this summation corresponds to a world-sheet $\mathrm{OPE}$, and can be represented by a Reggeon vertex operator. More directly, one finds,

$$
\mathcal{A}_{0}(s, t) \simeq\left(-\alpha^{\prime} s\right)^{1+\alpha^{\prime} t} \int_{0}^{\infty} d z z^{-2-\alpha^{\prime} t} e^{-z}=\Gamma\left(-1-\alpha^{\prime} t\right)\left(e^{-i \pi} \alpha^{\prime} s\right)^{1+\alpha^{\prime} t} .
$$

Consider next $\mathcal{A}_{0}(t, u)$ and $\mathcal{A}_{0}(u, s)$. For $\mathcal{A}_{0}(u, s)$, this corresponds to a fixed-angle limit and its contribution is exponentially suppressed. For $\mathcal{A}_{0}(t, u)$, it leads to $\Gamma(-1-$ $\left.\alpha^{\prime} t\right)\left(e^{-i \pi} \alpha^{\prime} u\right)^{1+\alpha^{\prime} t} \simeq \Gamma\left(-1-\alpha^{\prime} t\right)\left(\alpha^{\prime} s\right)^{1+\alpha^{\prime} t}$, leading to a total contribution that can be expressed as

$$
\mathcal{A}_{\mathrm{open}}(s, t) \simeq \pi \Gamma\left(\alpha^{\prime} t\right) \frac{\left(1+e^{-i \pi\left(1+\alpha^{\prime} t\right)}\right)}{\sin \pi\left(1+\alpha^{\prime} t\right)}\left(\alpha^{\prime} s\right)^{1+\alpha^{\prime} t} .
$$

In the physical region where $s>0$ and $t<0$, the discontinuity formula corresponds to

$$
\operatorname{Im} \mathcal{A}_{\text {open }}(s, t) \simeq \pi \Gamma\left(\alpha^{\prime} t\right)\left(\alpha^{\prime} s\right)^{1+\alpha^{\prime} t} .
$$

The same analysis can also be carried out for the closed-string amplitude. For large $s$ at fixed $t$, the region $w=O\left(s^{-1}\right)$ dominates, leading to Regge behavior

$$
\mathcal{A}_{\text {closed }} \sim 2 \pi \frac{\Gamma\left(-1-\alpha^{\prime} t / 4\right)}{\Gamma\left(2+\alpha^{\prime} t / 4\right)}\left(e^{-i \pi / 2} \alpha^{\prime} s / 4\right)^{2+\alpha^{\prime} t / 2} .
$$


Double-Regge limit for 5-point amplitude. We will be interested five-point string scattering, shown in figure 2, in the double-Regge limit, where we take $s=\left(p_{1}+p_{2}\right)^{2}$, $s_{1}=\left(p_{3}+p_{c}\right)^{2}$, and $s_{2}=\left(p_{5}+p_{c}\right)^{2}$, large, with $t_{1}=\left(p_{3}-p_{1}\right)^{2}, t_{2}=\left(p_{2}-p_{5}\right)^{2}$ and $\kappa \equiv \frac{s_{1} s_{2}}{s}$ fixed. Consider a planar order amplitude $V_{5}$ with planar ordering (13452). For exploring the double-Regge limit, it is best to use the Koba-Nielson representation

$$
V_{5}=\int_{0}^{1} \frac{d u}{u^{1+\alpha\left(t_{1}\right)}(1-u)^{1+\alpha\left(s_{1}\right)}} \int_{0}^{1} \frac{d v}{v^{1+\alpha\left(t_{2}\right)}(1-v)^{1+\alpha\left(s_{2}\right)}}(1-u v)^{\alpha(s)-\alpha\left(s_{1}\right)-\alpha\left(s_{2}\right)} .
$$

Now we take the limit $s_{1} \rightarrow-\infty, s_{2} \rightarrow-\infty$ and $s \rightarrow-\infty$, with $\kappa=s_{1} s_{2} / s$ fixed, to find that $V_{5} \simeq\left(-\alpha^{\prime} s_{1}\right)^{\alpha\left(t_{1}\right)} V_{c}\left(\alpha^{\prime} \kappa, t_{1}, t_{2}\right)\left(-\alpha^{\prime} s_{2}\right)^{\alpha\left(t_{2}\right)}$, where we have defined $\alpha(t)=1+\alpha^{\prime} t$ as well as a central vertex coupling

$$
V_{c}\left(t_{1}, t_{2}, x\right)=\int_{0}^{\infty} d y_{1} \int_{0}^{\infty} d y_{2} y_{1}^{-\alpha^{\prime} t_{1}-2} y_{2}^{-\alpha^{\prime} t_{2}-2} e^{-y_{1}-y_{2}+\frac{y_{1} y_{2}}{x}},
$$

with $x=\frac{s_{1} s_{2}}{\alpha^{\prime} s}$. This representation is valid for $\kappa<0$, and the physical region $\kappa>0$ is to be reached via analytic continuation. From eq. (D.10), one observes that $V_{c}\left(x, t_{1}, t_{2}\right)$ is real-analytic, with a branch-cut over $0<x<\infty$. For $x>0$, one finds that

$$
\begin{aligned}
\operatorname{Im} V_{c}\left(x, t_{1}, t_{2}\right) & =\frac{\pi x^{-\left(\alpha_{1}+\alpha_{2}+1\right)} e^{-x}}{\Gamma\left(\alpha_{1}+1\right) \Gamma\left(\alpha_{2}+1\right)} \int_{0}^{\infty} d u \int_{0}^{\infty} d v u^{\alpha_{1}} v^{\alpha_{2}} e^{-\left(u+v+\frac{u v}{x}\right)} \\
& =\pi e^{-x} \Psi\left(\alpha_{2}+1,-\alpha_{1}+\alpha_{2}+1 ; x\right)
\end{aligned}
$$

where $\Psi$ is the confluent hypergeometric function and we have also abbreviated $\alpha\left(t_{i}\right)$ by $\alpha_{i}, i=1,2$. Most importantly, for $x>0$ and $x \rightarrow \infty, \operatorname{Im} V_{c}$ vanishes exponentially,

$$
\operatorname{Im} V_{c}\left(x, t_{1}, t_{2}\right) \simeq \pi x^{-\alpha_{1}-\alpha_{2}-1} e^{-x}
$$

so that in this limit $V_{c}$ becomes real and factorizable, $V_{c}\left(x, t_{1}, t_{2}\right) \rightarrow \Gamma\left(\alpha_{1}\right) \Gamma\left(\alpha_{2}\right)$.

We have considered so far only a particular planar ordering for the amplitude; to obtain the full amplitude, we need to sum over all other orderings, each of which we expect to have a similar double-Regge limit. A similar expression holds for closed strings [59, 60]. In AdS/CFT, the central vertex takes on the form $V_{c}\left(\widetilde{t_{1}}, \widetilde{t_{2}}, \widetilde{x}\right)$ with all invariants redshifted [52] as appears in eq. (4.7).

The six-point string amplitude. From [56], the six-point amplitude depicted in figure 3 is given by

$$
\begin{aligned}
& V_{6}=\int_{0}^{1} \frac{d u}{u^{\alpha_{1}+1}(1-u)^{\alpha_{a \bar{c}}+1}} \int_{0}^{1} \frac{d v}{v^{\alpha_{2}+1}(1-v)^{\alpha_{\bar{b} c}+1}} \int_{0}^{1} \frac{d w}{w^{\alpha_{\bar{a} a \bar{c}}+1}(1-w)^{\alpha_{b \bar{b} c}+1}} \\
& \times(1-(u+v) w+u v w)^{\alpha_{a \bar{a} c}}\left[\frac{1-(u+v) w+u v w}{(1-u w)(1-v w)}\right]^{\alpha\left(M^{2}\right)-\alpha_{a \bar{c}}-\alpha_{b \bar{c}}} .
\end{aligned}
$$

In the double Regge limit, we have $s_{a \bar{c}} \simeq s_{\bar{a} c} \rightarrow-\infty, s_{\bar{b} \bar{c}} \simeq s_{\bar{b} c} \rightarrow-\infty$ with $M^{2}=\left(p_{a}+p_{b}-\right.$ $\left.p_{c}\right)^{2} \simeq s=\left(p_{a}+p_{b}\right)^{2}$, and $\kappa \equiv \frac{s_{a \bar{c}} s_{b \bar{c}}}{M^{2}}$ fixed. In this limit, the dominant contribution comes from $u=O\left(1 / s_{a \bar{c}}\right)$ and $v=O\left(1 / s_{b \bar{c}}\right)$, and one finds $V_{6} \rightarrow\left(-\alpha_{a \bar{c}}\right)^{\alpha_{1}} \mathcal{V}_{c \bar{c}}\left(-\alpha_{b \bar{c}}\right)^{\alpha_{2}}$, where

$$
\mathcal{V}_{c \bar{c}}=\int_{0}^{1} \frac{d y}{y^{\alpha \bar{a} a \bar{c}+1}(1-y)^{\alpha_{b \bar{b}}}+1} V_{c}\left(t_{1}, t_{2}, \frac{\alpha^{\prime} \kappa}{y(1-y)}\right)
$$


with the usual identification of invariants with it linear trajectory function, e.g., $\alpha(t)=\alpha^{\prime} t+1$. The discontinuity in $M^{2}$, which now enters through $\kappa$, is given by

$$
\operatorname{Im} \mathcal{V}_{c \bar{c}}\left(\kappa, t_{1}, t_{2}\right)=\int_{0}^{1} \frac{d y}{y^{\alpha_{\bar{a} a \bar{c}}+1}(1-y)^{\alpha_{b \bar{b} c}+1}} \operatorname{Im} V_{c}\left(t_{1}, t_{2}, \frac{\alpha^{\prime} \kappa}{y(1-y)}\right) .
$$

For external tachyons, at $t_{1}=t_{2}=0$ we have $\alpha_{\bar{a} a \bar{c}}(0)=\alpha_{b \bar{b} c}(0)=0$, with $\operatorname{Im} \mathcal{V}_{c \bar{c}}(0,0, \kappa)$ finite.

\section{E Fit validation and parameter stability}

Here we provide more details on the fits to data presented in section 5 , specifically with respect to implementing a cut-off and the stability of parameters; the discussion here will be focused on technical details, and physical interpretation will be left in section 5 and 6 .

\section{E.1 Power-law behavior}

As discussed above, the arguments of section 4 suggest that the cross section should behave as

$$
\frac{d^{3} \sigma}{d^{2} p_{\perp} d \eta} \sim p_{\perp}^{-2 \Delta}
$$

where $\Delta$ is the conformal weight of the particle mediating production in the bulk. This naively indicates that we should fit to data a power-law curve of the form

$$
\frac{d^{3} \sigma}{d^{2} p_{\perp} d \eta}=\frac{A}{p_{\perp}^{B}}
$$

where the overall normalization $A$ and exponent $B$ are floated.

However, this formula is only expected to be true asymptotically as $p_{\perp} \rightarrow \infty$. In general, there are expected to be small- $p_{\perp}$ effects that are not visible to our analysis. This can be easily seen by noticing that the cross section diverges as $p_{\perp} \rightarrow 0$. There are several ways one could imagine modifying eq. (E.1) to include these effects. One particularly obvious way to avoid these effects is to fit a sum of the power-law curve and some other curve to data; in this approach, the second curve is intended to model directly the low- $p_{T}$ physics. Such an approach was recently advocated in $[102,105]$. Although we are not interested here in this region we comment on it below in section E.2. Given that we are not interested in these non-universal effects at small momenta, we have no principled reason to prefer any one form of this low- $p_{\perp}$ curve over any other. Especially given that introducing such an extra curve would drastically increase the number of floated parameters, and hence potentially lead to overfitting, it is best to be more agnostic as to the form of the low- $p_{\perp}$ effects.

We will therefore consider simpler ways to remove low- $p_{\perp}$ effects. Perhaps the most obvious solution would be to simply introduce a lower cuttoff $p_{\min }$ on the allowed $p_{\perp}$, and therefore only fit to a subset of each data sample. Another approach is to allow a small offset in the momentum that appears in the power law curve, i.e. to fit a three-parameter curve of the form

$$
\frac{d^{3} \sigma}{d^{2} p_{\perp} d \eta}=\frac{A}{\left(p_{\perp}+C\right)^{B}}
$$




\begin{tabular}{|c|c|c|c|}
\hline$p_{\min } /(1 \mathrm{GeV})$ & $\mathrm{A} / 10\left(\mathrm{GeV}^{-2}\right)$ & $\mathrm{B}$ & $\chi^{2} / \mathrm{NDF}$ \\
\hline 0 & $0.0516 \pm 0.00687$ & $5.02 \pm 0.164$ & 51.2 \\
\hline 0.5 & $0.0575 \pm 0.00718$ & $5.15 \pm 0.148$ & 29.8 \\
\hline 1.0 & $0.0943 \pm 0.0140$ & $5.60 \pm 0.139$ & 3.21 \\
\hline 1.5 & $0.153 \pm 0.0585$ & $5.88 \pm 0.231$ & 0.135 \\
\hline 2.0 & $0.183 \pm 0.131$ & $5.97 \pm 0.368$ & 0.0412 \\
\hline 2.5 & $0.199 \pm 0.247$ & $6.01 \pm 0.578$ & 0.0337 \\
\hline 3.0 & $0.205 \pm 0.291$ & $6.027 \pm 0.646$ & 0.0316 \\
\hline 3.5 & $0.218 \pm 0.348$ & $6.05 \pm 0.712$ & 0.0258 \\
\hline 4.0 & $0.233 \pm 0.416$ & $6.07 \pm 0.770$ & 0.0189 \\
\hline 4.5 & $0.253 \pm 0.518$ & $6.10 \pm 0.846$ & 0.0127 \\
\hline 5.0 & $0.150 \pm 0.736$ & $5.93 \pm 1.70$ & 0.000621 \\
\hline
\end{tabular}

Table 2. Fitted values of parameters in eq. (E.2) for the ATLAS dataset at $\sqrt{s}=8 \mathrm{TeV}$ [94].

instead of the two-parameter form presented in eq. (E.2). This form has two advantages. First, for $C>0$, the numerical singularity at $p_{\perp}=0$ is directly removed; additionally, as $p_{\perp} \rightarrow \infty$, it is readily seen to agree with eq. (E.1). One could imagine adding in a lower cutoff to this form of the curve as well.

Without a handle on the small- $p_{\perp}$ physics, we have no theoretical reason to prefer one of these approaches over the other. We will therefore fit both forms to data, both with and without a cutoff, and choose the approach that gives the quantitatively best overall results, as quantified by $\chi^{2} / \mathrm{NDF}$. In the following pages, we will present a thorough evaluation of these two methods. For each of the five datasets discussed in the main text, we will present the results of twenty-two fits to data, corresponding to eleven different cutoffs $p_{\min }$ for each of the two fit functions in eqs. (E.2) and (E.3). We will also display some characteristic plots, to facilitate a visual analysis of the results.

From the fit results in tables 2 through 11, we can immediately exclude the proposal to fit eq. (E.2) directly to data. For all values of the cutoff tested, the $\chi^{2} /$ NDF is unacceptable, being extremely high at small or no cutoff, and then rapidly falling to below one at large cutoff. This leads us to consider instead eq. (E.3), and leaves only the question of whether or not to institute a cutoff, and if so what value of the cutoff to use. For much the same reasons as above, we dispense with the possibility of a large cutoff. For cutoffs between 0 and $1.5 \mathrm{GeV}$, the gains in $\chi^{2} / \mathrm{NDF}$ are minimal for removing the low- $p_{\perp}$ data. Thus, to be conservative, and to minimize the overall statistical uncertainties, we will fit eq. (E.3) to data directly, without a cutoff. These are the results given in section 5 . 


\begin{tabular}{|c|c|c|c|c|}
\hline$p_{\min } /(1 \mathrm{GeV})$ & $\mathrm{A} / 10\left(\mathrm{GeV}^{-2}\right)$ & $\mathrm{B}$ & $\mathrm{C} /(1 \mathrm{GeV})$ & $\chi^{2} / \mathrm{NDF}$ \\
\hline 0 & $4.46 \pm 2.60$ & $7.04 \pm 0.264$ & $1.07 \pm 0.123$ & 1.19 \\
\hline 0.5 & $3.81 \pm 2.98$ & $6.98 \pm 0.326$ & $1.03 \pm 0.182$ & 1.08 \\
\hline 1.0 & $1.16 \pm 1.72$ & $6.57 \pm 0.554$ & $0.689 \pm 0.405$ & 0.279 \\
\hline 1.5 & $0.387 \pm 1.06$ & $6.21 \pm 0.959$ & $0.311 \pm 0.917$ & 0.0314 \\
\hline 2.0 & $0.309 \pm 1.22$ & $6.14 \pm 1.29$ & $0.212 \pm 1.573$ & 0.0255 \\
\hline 2.5 & $0.369 \pm 2.15$ & $6.19 \pm 1.74$ & $0.312 \pm 2.89$ & 0.0241 \\
\hline 3.0 & $0.448 \pm 3.10$ & $6.24 \pm 1.97$ & $0.429 \pm 3.74$ & 0.0228 \\
\hline 3.5 & $0.417 \pm 3.76$ & $6.22 \pm 2.47$ & $0.383 \pm 5.29$ & 0.0228 \\
\hline 4.0 & $0.191 \pm 2.03$ & $6.02 \pm 2.87$ & $-0.126 \pm 6.64$ & 0.0187 \\
\hline 4.5 & $0.0465 \pm 0.483$ & $5.66 \pm 2.81$ & $-1.09 \pm 6.72$ & 0.00502 \\
\hline 5.0 & $0.325 \pm 5.36$ & $6.13 \pm 4.13$ & $0.629 \pm 13.7$ & 0.000189 \\
\hline
\end{tabular}

Table 3. Fitted values of parameters in eq. (E.3) for the ATLAS dataset at $\sqrt{s}=8 \mathrm{TeV}$ [94].

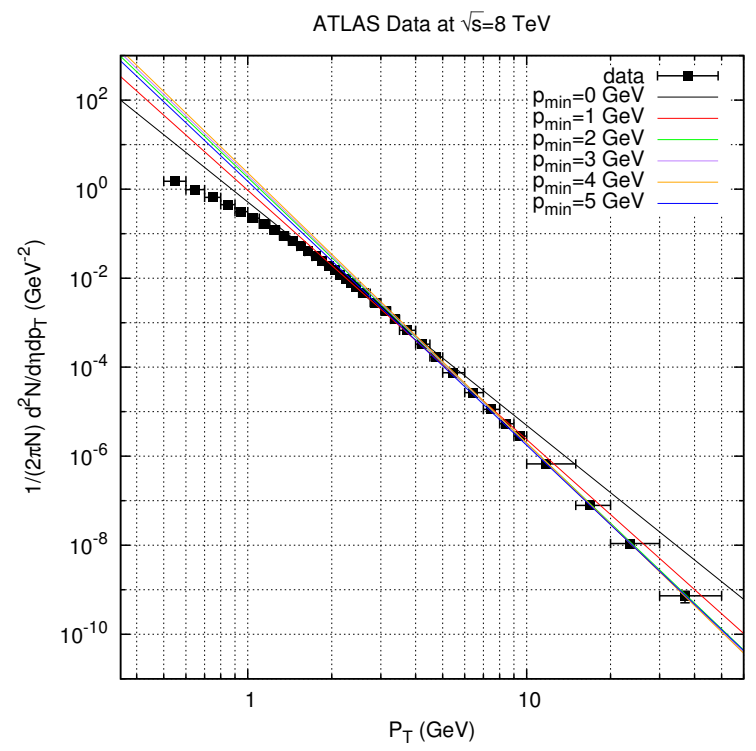

(a)

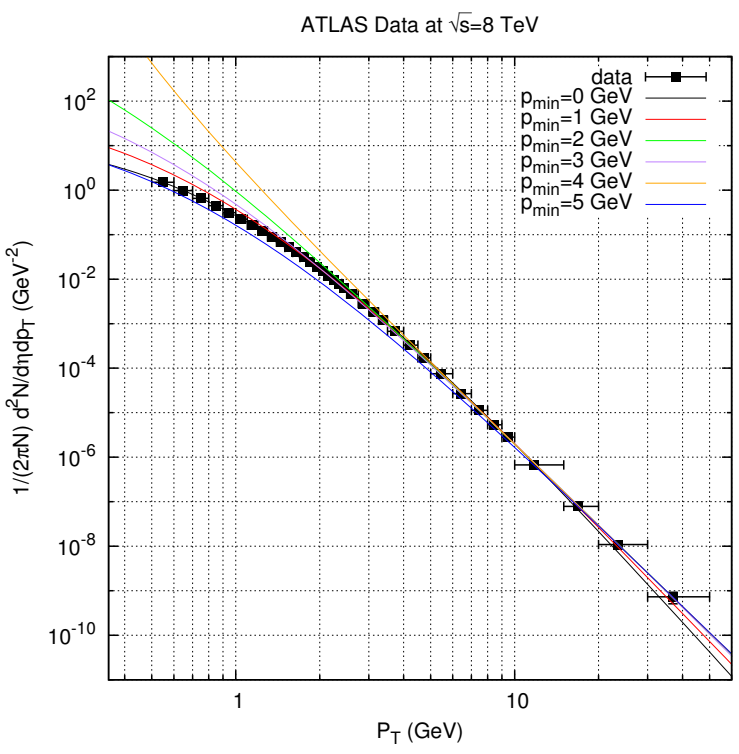

(b)

Figure 10. Fit of the two parameter ansatz in eq. (E.2) (left) and the three parameter ansatz in eq. (E.3) to the ATLAS dataset at $\sqrt{s}=8 \mathrm{TeV}[94]$ with various data cutoffs. 


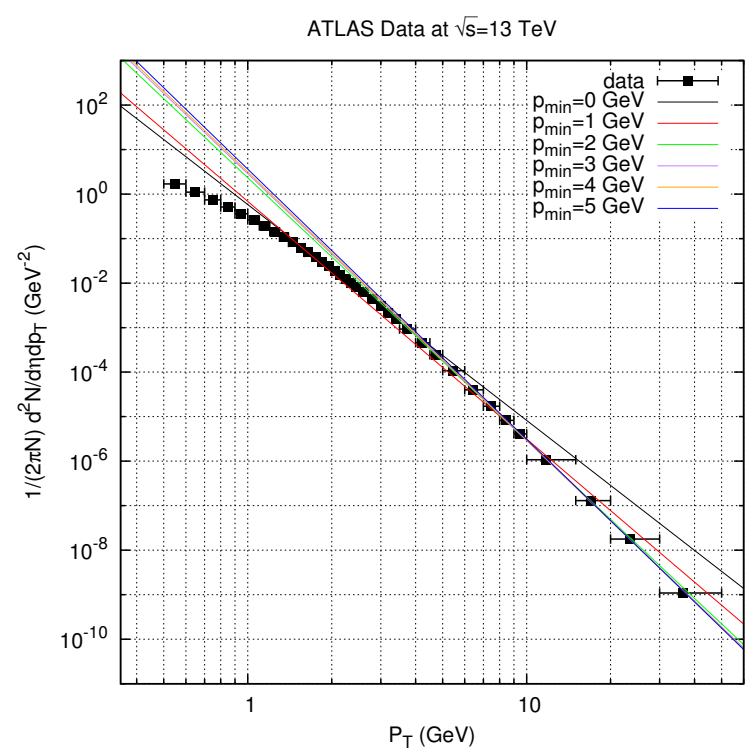

(a)

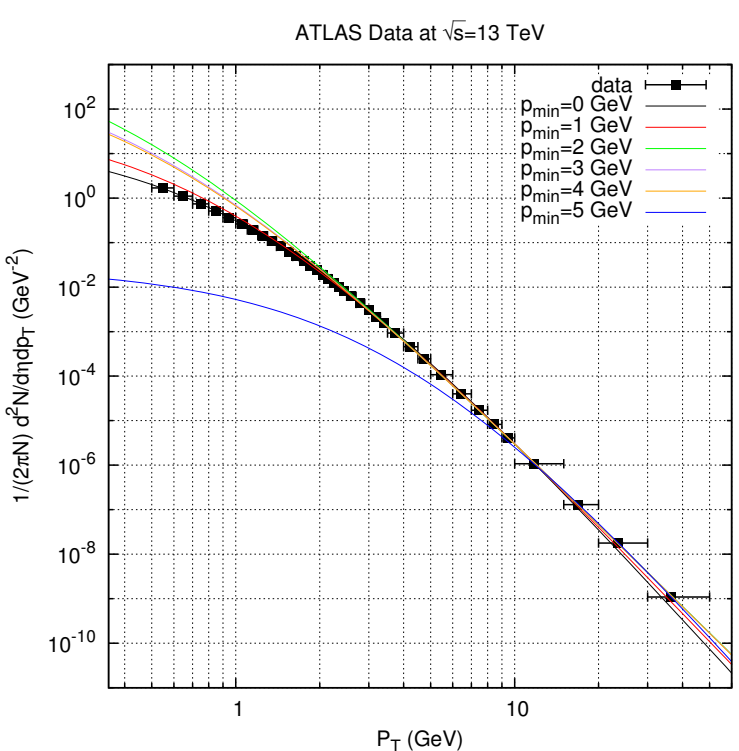

(b)

Figure 11. Fit of the two parameter ansatz in eq. (E.2) (left) and the three parameter ansatz in eq. (E.3) to the ATLAS dataset at $\sqrt{s}=13 \mathrm{TeV}$ [93] with various data cutoffs.

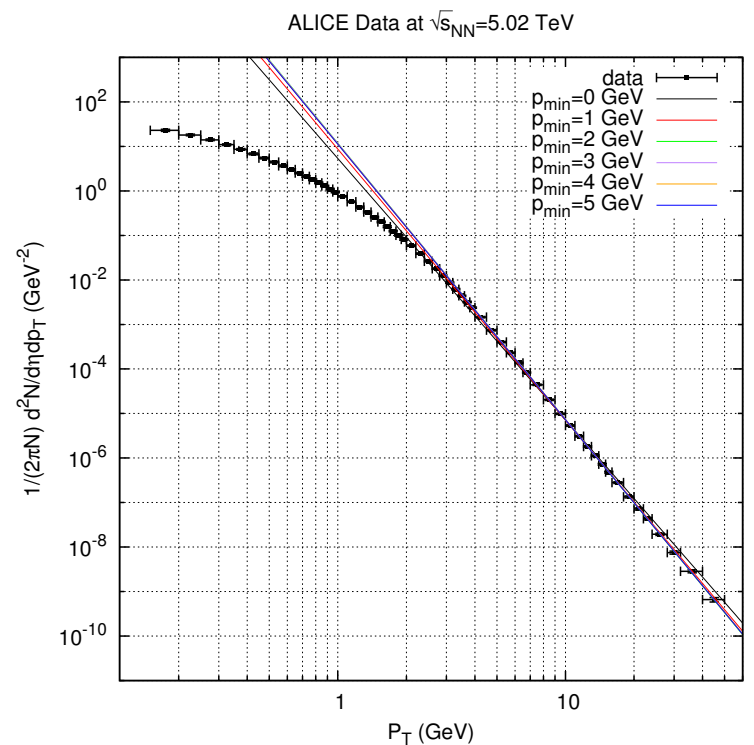

(a)

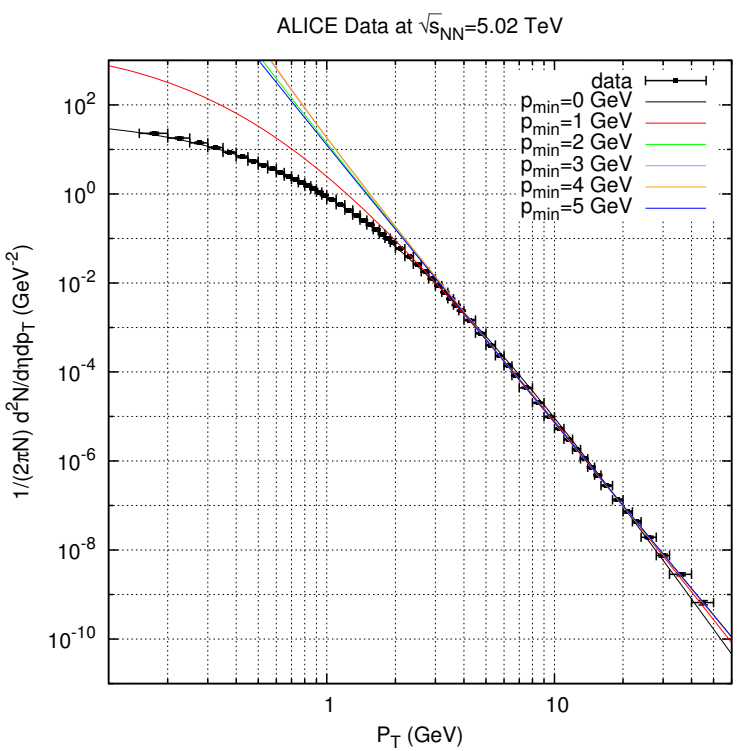

(b)

Figure 12. Fit of the two parameter ansatz in eq. (E.2) (left) and the three parameter ansatz in eq. (E.3) to the ALICE dataset at $\sqrt{s}=5.02 \mathrm{TeV}$ in the $|\eta|<0.3$ bin [95] with various data cutoffs. 


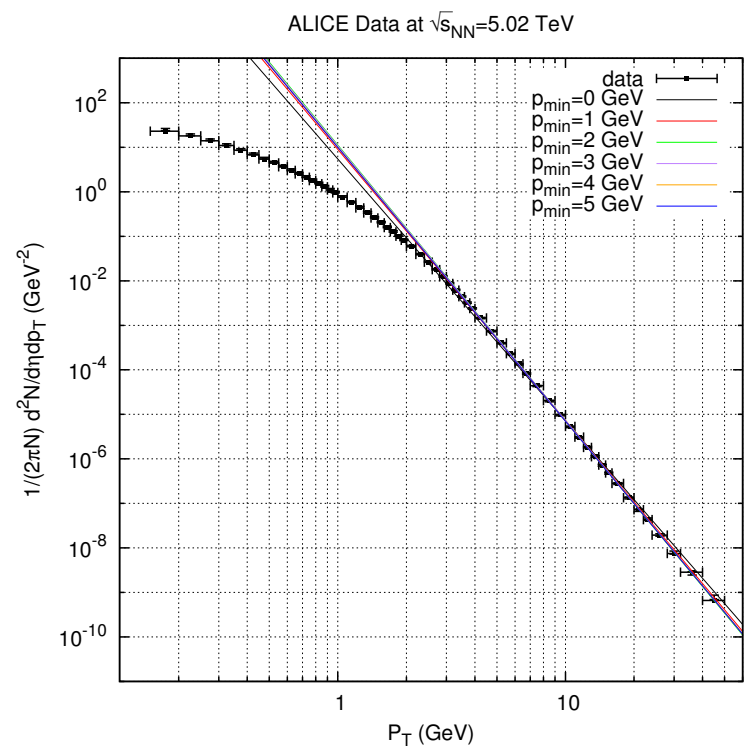

(a)

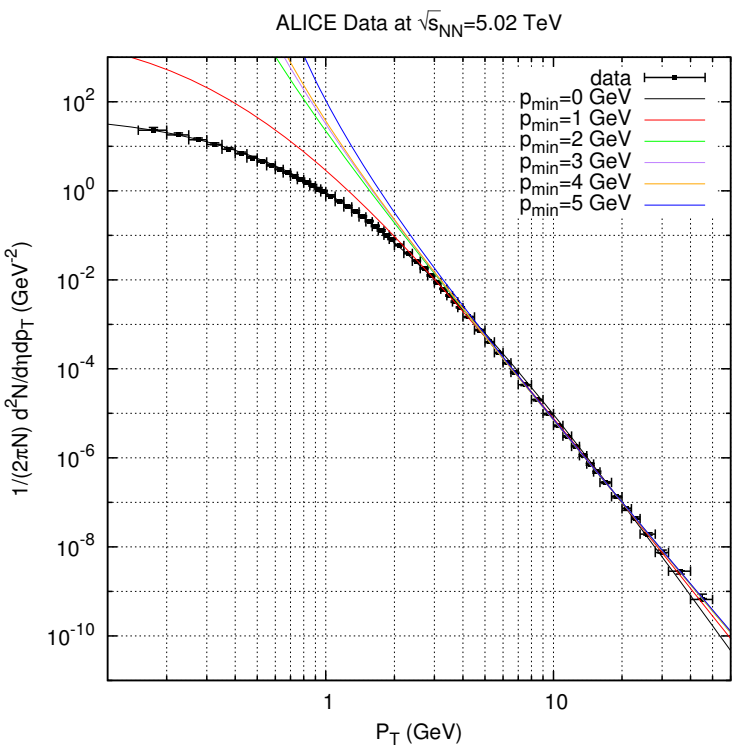

(b)

Figure 13. Fit of the two parameter ansatz in eq. (E.2) (left) and the three parameter ansatz in eq. (E.3) to the ALICE dataset at $\sqrt{s}=5.02 \mathrm{TeV}$ in the $-0.8<\eta<-0.3$ bin [95] with various data cutoffs.

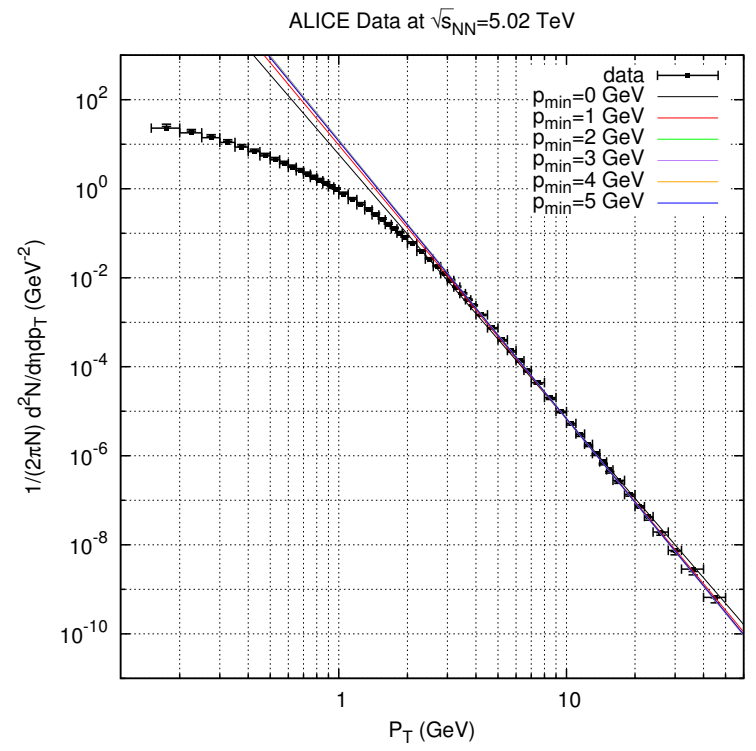

(a)

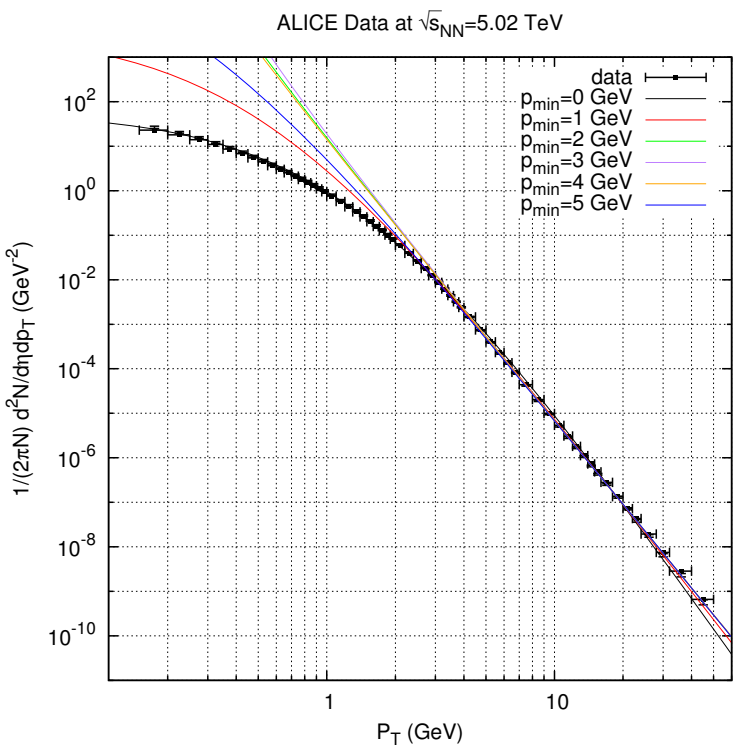

(b)

Figure 14. Fit of the two parameter ansatz in eq. (E.2) (left) and the three parameter ansatz in eq. (E.3) to the ALICE dataset at $\sqrt{s}=5.02 \mathrm{TeV}$ in the $-1.3<\eta<-0.8$ bin [95] with various data cutoffs. 


\begin{tabular}{|c|c|c|c|}
\hline$p_{\min } /(1 \mathrm{GeV})$ & $\mathrm{A} / 10\left(\mathrm{GeV}^{-2}\right)$ & $\mathrm{B}$ & $\chi^{2} / \mathrm{NDF}$ \\
\hline 0 & $0.0583 \pm 0.0070$ & $4.85 \pm 0.143$ & 54.0 \\
\hline 0.5 & $0.0648 \pm 0.0076$ & $4.97 \pm 0.137$ & 32.2 \\
\hline 1.0 & $-156 . \pm 0.372$ & $-207 . \pm 9.29$ & 0.361 \\
\hline 1.5 & $0.186 \pm 0.063$ & $5.79 \pm 0.216$ & 0.120 \\
\hline 2.0 & $0.234 \pm 0.170$ & $5.90 \pm 0.371$ & 0.0489 \\
\hline 2.5 & $0.270 \pm 0.339$ & $5.96 \pm 0.583$ & 0.0272 \\
\hline 3.0 & $0.286 \pm 0.405$ & $5.99 \pm 0.646$ & 0.0203 \\
\hline 3.5 & $0.300 \pm 0.484$ & $6.01 \pm 0.717$ & 0.0166 \\
\hline 4.0 & $0.314 \pm 0.574$ & $6.025 \pm 0.790$ & 0.0141 \\
\hline 4.5 & $0.305 \pm 0.661$ & $6.01 \pm 0.897$ & 0.0135 \\
\hline 5.0 & $0.364 \pm 2.03$ & $6.07 \pm 1.90$ & 0.0125 \\
\hline
\end{tabular}

Table 4. Fitted values of parameters in eq. (E.2) for the ATLAS dataset at $\sqrt{s}=13 \mathrm{TeV}$ [93].

\begin{tabular}{|c|c|c|c|c|}
\hline$p_{\min } /(1 \mathrm{GeV})$ & $\mathrm{A} / 10\left(\mathrm{GeV}^{-2}\right)$ & $\mathrm{B}$ & $\mathrm{C} /(1 \mathrm{GeV})$ & $\chi^{2} / \mathrm{NDF}$ \\
\hline 0 & $5.77 \pm 3.39$ & $6.96 \pm 0.265$ & $1.12 \pm 0.126$ & 0.852 \\
\hline 0.5 & $5.22 \pm 4.10$ & $6.93 \pm 0.328$ & $1.09 \pm 0.185$ & 0.784 \\
\hline 1.0 & $2.07 \pm 2.95$ & $6.61 \pm 0.536$ & $0.820 \pm 0.396$ & 0.223 \\
\hline 1.5 & $0.667 \pm 1.88$ & $6.23 \pm 0.979$ & $0.431 \pm 0.947$ & 0.0178 \\
\hline 2.0 & $0.544 \pm 2.30$ & $6.17 \pm 1.38$ & $0.341 \pm 1.69$ & 0.0138 \\
\hline 2.5 & $0.621 \pm 3.69$ & $6.21 \pm 1.78$ & $0.418 \pm 2.93$ & 0.0125 \\
\hline 3.0 & $0.634 \pm 4.33$ & $6.21 \pm 1.97$ & $0.429 \pm 3.65$ & 0.0124 \\
\hline 3.5 & $0.670 \pm 5.56$ & $6.23 \pm 2.31$ & $0.463 \pm 4.78$ & 0.0124 \\
\hline 4.0 & $0.652 \pm 6.99$ & $6.22 \pm 2.90$ & $0.445 \pm 6.53$ & 0.0124 \\
\hline 4.5 & $2.18 \pm 27.76$ & $6.53 \pm 3.31$ & $1.26 \pm 8.16$ & 0.00694 \\
\hline 5.0 & $45.2 \pm 790$. & $7.24 \pm 4.24$ & $3.80 \pm 13.9$ & 0.000184 \\
\hline
\end{tabular}

Table 5. Fitted values of parameters in eq. (E.3) for the ATLAS dataset at $\sqrt{s}=13 \mathrm{TeV}$ [94]. 


\begin{tabular}{|c|c|c|c|}
\hline$p_{\min } /(1 \mathrm{GeV})$ & $\mathrm{A} / 10\left(\mathrm{GeV}^{-2}\right)$ & $\mathrm{B}$ & $\chi^{2} / \mathrm{NDF}$ \\
\hline 0 & $0.535 \pm 0.0638$ & $5.87 \pm 0.0710$ & 352. \\
\hline 0.5 & $0.528 \pm 0.05674$ & $5.85 \pm 0.0662$ & 91.1 \\
\hline 1.0 & $0.868 \pm 0.110$ & $6.09 \pm 0.0652$ & 5.73 \\
\hline 1.5 & $1.00 \pm 0.151$ & $6.15 \pm 0.0720$ & 0.771 \\
\hline 2.0 & $1.18 \pm 0.338$ & $6.21 \pm 0.116$ & 0.100 \\
\hline 2.5 & $1.19 \pm 0.386$ & $6.21 \pm 0.129$ & 0.0989 \\
\hline 3.0 & $1.17 \pm 0.462$ & $6.20 \pm 0.152$ & 0.0945 \\
\hline 3.5 & $1.15 \pm 0.647$ & $6.20 \pm 0.208$ & 0.0890 \\
\hline 4.0 & $1.13 \pm 0.690$ & $6.19 \pm 0.222$ & 0.0865 \\
\hline 4.5 & $1.11 \pm 0.735$ & $6.19 \pm 0.240$ & 0.0807 \\
\hline 5.0 & $1.12 \pm 0.767$ & $6.19 \pm 0.246$ & 0.0802 \\
\hline
\end{tabular}

Table 6. Fitted values of parameters in eq. (E.2) for the ALICE dataset at $\sqrt{s}=5.02 \mathrm{TeV}$ in the $|\eta|<0.3$ bin [95].

\begin{tabular}{|c|c|c|c|c|}
\hline$p_{\min } /(1 \mathrm{GeV})$ & $\mathrm{A} / 10\left(\mathrm{GeV}^{-2}\right)$ & $\mathrm{B}$ & $\mathrm{C} /(1 \mathrm{GeV})$ & $\chi^{2} / \mathrm{NDF}$ \\
\hline 0 & $38.5 \pm 8.27$ & $7.23 \pm 0.0853$ & $1.32 \pm 0.0445$ & 14.7 \\
\hline 0.5 & $22.0 \pm 9.59$ & $7.07 \pm 0.146$ & $1.14 \pm 0.120$ & 8.78 \\
\hline 1.0 & $3.89 \pm 2.99$ & $6.55 \pm 0.241$ & $0.525 \pm 0.261$ & 1.68 \\
\hline 1.5 & $1.86 \pm 1.79$ & $6.34 \pm 0.298$ & $0.232 \pm 0.354$ & 0.334 \\
\hline 2.0 & $1.08 \pm 1.63$ & $6.18 \pm 0.444$ & $-0.0403 \pm 0.704$ & 0.0971 \\
\hline 2.5 & $0.991 \pm 1.76$ & $6.16 \pm 0.514$ & $-0.0894 \pm 0.886$ & 0.0893 \\
\hline 3.0 & $0.985 \pm 2.05$ & $6.16 \pm 0.587$ & $-0.0926 \pm 1.12$ & 0.0882 \\
\hline 3.5 & $0.946 \pm 2.84$ & $6.15 \pm 0.808$ & $-0.124 \pm 1.92$ & 0.0853 \\
\hline 4.0 & $0.979 \pm 3.34$ & $6.16 \pm 0.904$ & $-0.0978 \pm 2.28$ & 0.0850 \\
\hline 4.5 & $1.15 \pm 4.51$ & $6.20 \pm 1.02$ & $0.0252 \pm 2.74$ & 0.0807 \\
\hline 5.0 & $1.09 \pm 4.98$ & $6.18 \pm 1.18$ & $-0.0206 \pm 3.33$ & 0.0802 \\
\hline
\end{tabular}

Table 7. Fitted values of parameters in eq. (E.3) for the ALICE dataset at $\sqrt{s}=5.02 \mathrm{TeV}$ in the $|\eta|<0.3$ bin [95]. 


\begin{tabular}{|c|c|c|c|}
\hline$p_{\min } /(1 \mathrm{GeV})$ & $\mathrm{A} / 10\left(\mathrm{GeV}^{-2}\right)$ & $\mathrm{B}$ & $\chi^{2} / \mathrm{NDF}$ \\
\hline 0 & $0.545 \pm 0.0635$ & $5.88 \pm 0.0694$ & 348. \\
\hline 0.5 & $0.541 \pm 0.0570$ & $5.86 \pm 0.0649$ & 88.0 \\
\hline 1.0 & $0.879 \pm 0.110$ & $6.09 \pm 0.06428$ & 5.12 \\
\hline 1.5 & $1.00 \pm 0.150$ & $6.15 \pm 0.0709$ & 0.603 \\
\hline 2.0 & $1.12 \pm 0.316$ & $6.19 \pm 0.114$ & 0.198 \\
\hline 2.5 & $1.11 \pm 0.356$ & $6.18 \pm 0.127$ & 0.192 \\
\hline 3.0 & $1.08 \pm 0.419$ & $6.17 \pm 0.149$ & 0.177 \\
\hline 3.5 & $1.02 \pm 0.567$ & $6.15 \pm 0.204$ & 0.158 \\
\hline 4.0 & $1.00 \pm 0.600$ & $6.15 \pm 0.219$ & 0.151 \\
\hline 4.5 & $0.981 \pm 0.638$ & $6.14 \pm 0.235$ & 0.144 \\
\hline 5.0 & $0.993 \pm 0.699$ & $6.15 \pm 0.252$ & 0.142 \\
\hline
\end{tabular}

Table 8. Fitted values of parameters in eq. (E.2) for the ALICE dataset at $\sqrt{s}=5.02 \mathrm{TeV}$ in the $-0.8<\eta<-0.3$ bin [95].

\begin{tabular}{|c|c|c|c|c|}
\hline$p_{\min } /(1 \mathrm{GeV})$ & $\mathrm{A} / 10\left(\mathrm{GeV}^{-2}\right)$ & $\mathrm{B}$ & $\mathrm{C} /(1 \mathrm{GeV})$ & $\chi^{2} / \mathrm{NDF}$ \\
\hline 0 & $37.6 \pm 7.97$ & $7.22 \pm 0.0841$ & $1.30 \pm 0.0439$ & 17.6 \\
\hline 0.5 & $19.6 \pm 8.33$ & $7.03 \pm 0.142$ & $1.10 \pm 0.117$ & 10.4 \\
\hline 1.0 & $3.16 \pm 2.37$ & $6.49 \pm 0.236$ & $0.448 \pm 0.256$ & 2.09 \\
\hline 1.5 & $1.44 \pm 1.34$ & $6.26 \pm 0.289$ & $0.135 \pm 0.343$ & 0.446 \\
\hline 2.0 & $0.819 \pm 1.19$ & $6.10 \pm 0.427$ & $-0.149 \pm 0.678$ & 0.152 \\
\hline 2.5 & $0.754 \pm 1.30$ & $6.07 \pm 0.497$ & $-0.196 \pm 0.862$ & 0.144 \\
\hline 3.0 & $0.735 \pm 1.47$ & $6.07 \pm 0.564$ & $-0.212 \pm 1.09$ & 0.142 \\
\hline 3.5 & $0.691 \pm 1.95$ & $6.05 \pm 0.758$ & $-0.256 \pm 1.83$ & 0.142 \\
\hline 4.0 & $0.708 \pm 2.30$ & $6.06 \pm 0.858$ & $-0.238 \pm 2.20$ & 0.142 \\
\hline 4.5 & $0.766 \pm 2.81$ & $6.08 \pm 0.956$ & $-0.177 \pm 2.62$ & 0.140 \\
\hline 5.0 & $0.595 \pm 2.53$ & $6.01 \pm 1.10$ & $-0.381 \pm 3.15$ & 0.131 \\
\hline
\end{tabular}

Table 9. Fitted values of parameters in eq. (E.3) for the ALICE dataset at $\sqrt{s}=5.02 \mathrm{TeV}$ in the $-0.8<\eta<-0.3$ bin [95]. 


\begin{tabular}{|c|c|c|c|}
\hline$p_{\min } /(1 \mathrm{GeV})$ & $\mathrm{A} / 10\left(\mathrm{GeV}^{-2}\right)$ & $\mathrm{B}$ & $\chi^{2} / \mathrm{NDF}$ \\
\hline 0 & $0.592 \pm 0.0708$ & $5.94 \pm 0.0708$ & 347. \\
\hline 0.5 & $0.580 \pm 0.0627$ & $5.91 \pm 0.0665$ & 89.5 \\
\hline 1.0 & $0.949 \pm 0.121$ & $6.15 \pm 0.0655$ & 5.44 \\
\hline 1.5 & $1.09 \pm 0.166$ & $6.21 \pm 0.0724$ & 0.745 \\
\hline 2.0 & $1.25 \pm 0.363$ & $6.26 \pm 0.117$ & 0.213 \\
\hline 2.5 & $1.25 \pm 0.414$ & $6.26 \pm 0.131$ & 0.213 \\
\hline 3.0 & $1.241 \pm 0.498$ & $6.26 \pm 0.154$ & 0.211 \\
\hline 3.5 & $1.208 \pm 0.693$ & $6.25 \pm 0.211$ & 0.207 \\
\hline 4.0 & $1.19 \pm 0.740$ & $6.24 \pm 0.226$ & 0.204 \\
\hline 4.5 & $1.19 \pm 0.796$ & $6.24 \pm 0.243$ & 0.204 \\
\hline 5.0 & $1.16 \pm 0.854$ & $6.23 \pm 0.263$ & 0.199 \\
\hline
\end{tabular}

Table 10. Fitted values of parameters in eq. (E.2) for the ALICE dataset at $\sqrt{s}=5.02 \mathrm{TeV}$ in the $-1.3<\eta<-0.8$ bin [95].

\begin{tabular}{|c|c|c|c|c|}
\hline$p_{\min } /(1 \mathrm{GeV})$ & $\mathrm{A} / 10\left(\mathrm{GeV}^{-2}\right)$ & $\mathrm{B}$ & $\mathrm{C} /(1 \mathrm{GeV})$ & $\chi^{2} / \mathrm{NDF}$ \\
\hline 0 & $43.0 \pm 9.29$ & $7.30 \pm 0.0861$ & $1.31 \pm 0.0442$ & 16.7 \\
\hline 0.5 & $24.5 \pm 10.7$ & $7.13 \pm 0.147$ & $1.13 \pm 0.119$ & 9.29 \\
\hline 1.0 & $4.01 \pm 3.12$ & $6.60 \pm 0.244$ & $0.498 \pm 0.262$ & 1.82 \\
\hline 1.5 & $1.87 \pm 1.82$ & $6.37 \pm 0.303$ & $0.199 \pm 0.355$ & 0.424 \\
\hline 2.0 & $1.13 \pm 1.74$ & $6.23 \pm 0.453$ & $-0.0483 \pm 0.709$ & 0.209 \\
\hline 2.5 & $1.07 \pm 1.96$ & $6.22 \pm 0.528$ & $-0.0768 \pm 0.902$ & 0.206 \\
\hline 3.0 & $1.05 \pm 2.24$ & $6.21 \pm 0.601$ & $-0.0892 \pm 1.14$ & 0.205 \\
\hline 3.5 & $1.07 \pm 3.25$ & $6.21 \pm 0.822$ & $-0.0805 \pm 1.92$ & 0.205 \\
\hline 4.0 & $1.14 \pm 3.94$ & $6.23 \pm 0.918$ & $-0.0332 \pm 2.28$ & 0.204 \\
\hline 4.5 & $1.19 \pm 4.77$ & $6.24 \pm 1.04$ & $0.00394 \pm 2.74$ & 0.204 \\
\hline 5.0 & $1.52 \pm 6.94$ & $6.30 \pm 1.17$ & $0.195 \pm 3.25$ & 0.196 \\
\hline
\end{tabular}

Table 11. Fitted values of parameters in eq. (E.3) for the ALICE dataset at $\sqrt{s}=5.02 \mathrm{TeV}$ in the $-1.3<\eta<-0.8$ bin [95]. 


\begin{tabular}{|c|c|c|c|}
\hline Dataset & \multicolumn{3}{|c|}{$A \exp (-x / B)$} \\
\hline & $\mathrm{A} / 10\left(\mathrm{GeV}^{-} 2\right)$ & $\mathrm{B}(\mathrm{GeV})$ & $\chi^{2} / \mathrm{NDF}$ \\
\hline ATLAS: $\sqrt{s}=13 \mathrm{TeV}[93]$ & $0.110 \pm 0.00306$ & $0.268 \pm 0.00636$ & 0.00381 \\
\hline ATLAS: $\sqrt{s}=8 \mathrm{TeV}[94]$ & $0.107 \pm 0.00292$ & $0.261 \pm 0.00579$ & 0.00312 \\
\hline ALICE $|\eta|<0.3[95]$ & $0.173 \pm 0.000271$ & $0.205 \pm 0.00136$ & 2.35 \\
\hline ALICE $-0.8<\eta<-0.3[95]$ & $0.179 \pm 0.000425$ & $0.194 \pm 0.00193$ & 4.48 \\
\hline ALICE $-1.3<\eta<-0.8[95]$ & $0.182 \pm 0.000335$ & $0.193 \pm 0.00151$ & 4.31 \\
\hline Dataset & \multicolumn{3}{|c|}{$A \exp \left(-x^{2} / B\right)$} \\
\hline ATLAS: $\sqrt{s}=13 \mathrm{TeV}[93]$ & $\mathrm{A} / 10\left(\mathrm{GeV}^{-} 2\right)$ & $\mathrm{B}(\mathrm{GeV})$ & $\chi^{2} / \mathrm{NDF}$ \\
\hline ATLAS: $\sqrt{s}=8 \mathrm{TeV}[94]$ & $0.0482 \pm 0.00489$ & $0.429 \pm 0.0300$ & 0.0350 \\
\hline ALICE $|\eta|<0.3[95]$ & $0.147 \pm 0.000967$ & $0.114 \pm 0.00688$ & 314 \\
\hline ALICE $-0.8<\eta<-0.3[95]$ & $0.152 \pm 0.00105$ & $0.106 \pm 0.00681$ & 517 \\
\hline ALICE $-1.3<\eta<-0.8[95]$ & $0.155 \pm 0.00101$ & $0.104 \pm 0.00631$ & 612 \\
\hline
\end{tabular}

Table 12. Fits of eq. (E.4) and eq. (E.5) to small $p_{T}<1 \mathrm{GeV}$ data. In the last three entries, the colors correspond to the color scheme used in e.g. figure 5 .

\section{E.2 Small $p_{T}$}

Here we comment on the small $p_{T}$ behavior. We are focused here on $p_{T}<1 \mathrm{GeV}$ where confinement effects, ekonalization, and saturation effects should all play a major roll. From the AdS/CFT perspective, many models display Regge phenomenology that are insensitive to these details at large enough energy scales [30, 91].

Although we are not focused on small $p_{T}$ behavior, we can however comment on two possible predictions. The first is that of flat space string theory which predicts a fall off

$$
d \sigma \sim A e^{-p_{T}^{2} / B}
$$

Recently it was advocated that a two parameter model applies at small $p_{T}[102,105]$ of the form

$$
d \sigma \sim A e^{-p_{T} / B}
$$

The results for these small $p_{T}$ fits can be seen in figure 15 and in table 12, although we caution that the limited amount of data in this region can lead to over-fitting. 


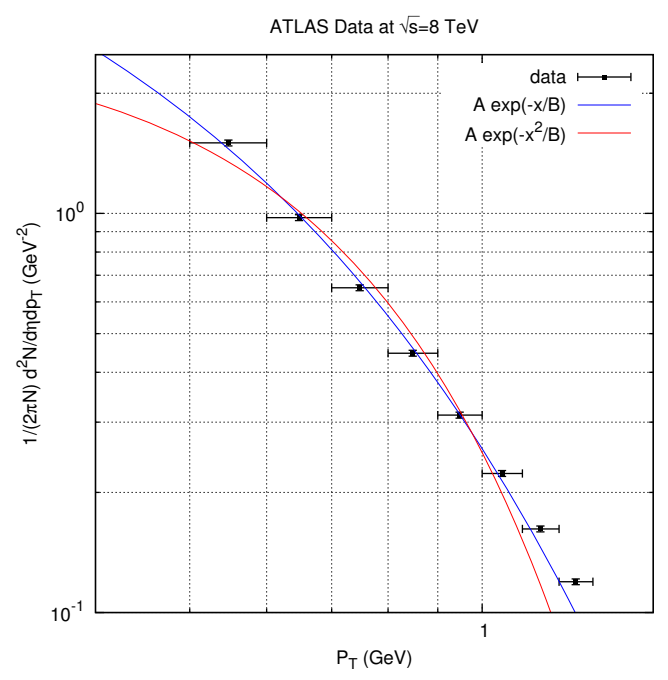

(a)

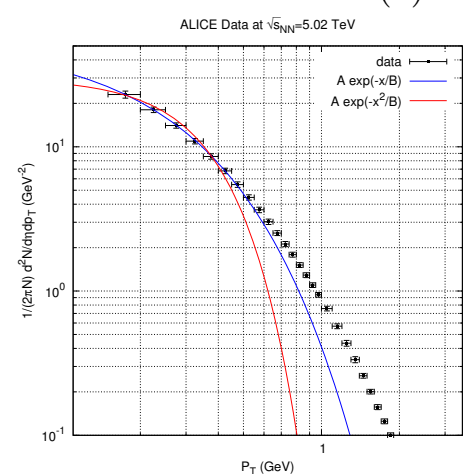

(c)

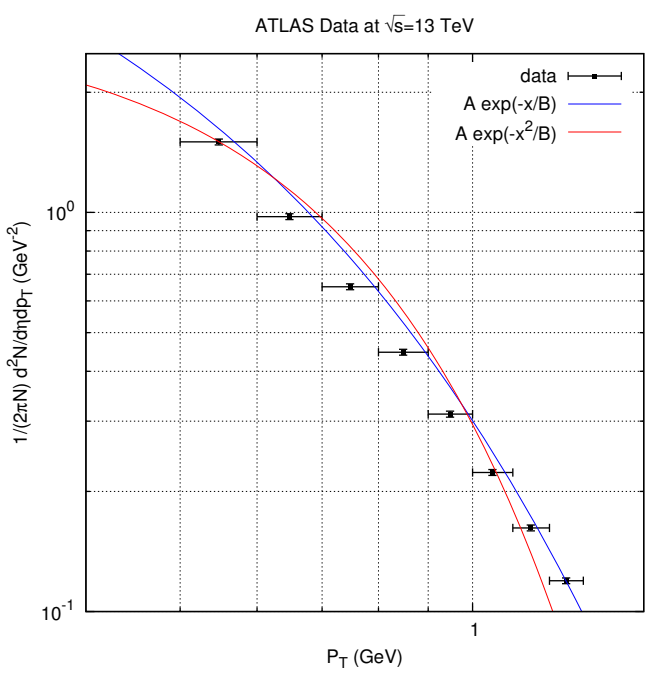

(b)

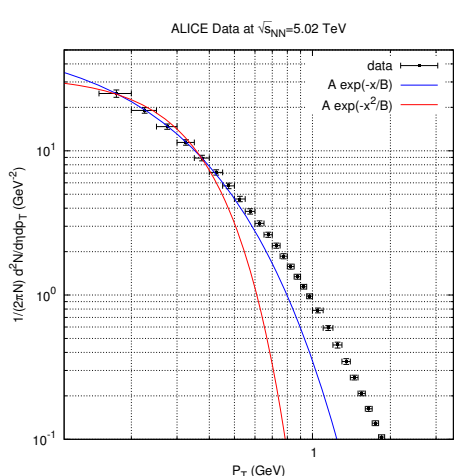

(d)

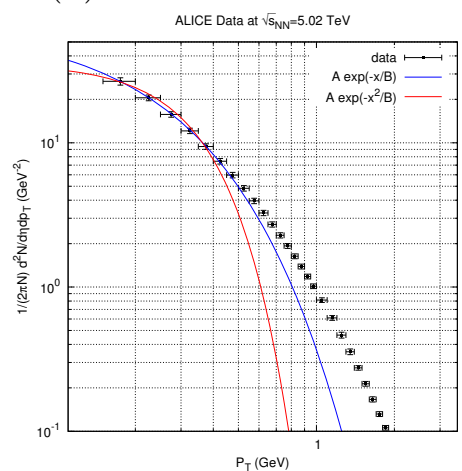

(e)

Figure 15. Fits of $A \exp (-x / B)$ and $A \exp \left(-x^{2} / B\right)$ to all data below $p_{T}=1 \mathrm{GeV}$ for the ATLAS $\sqrt{s}=8 \mathrm{TeV}$ data set (a), the ATLAS $\sqrt{s}=13 \mathrm{TeV}$ data set (b), the ALICE $\sqrt{s_{N N}}=5.02 \mathrm{TeV}|\eta|<$ 0.3 data set (c), the ALICE $\sqrt{s_{N N}}=5.02 \mathrm{TeV}-0.8<\eta<-0.3$ data set (d), and the ALICE $\sqrt{s_{N N}}=5.02 \mathrm{TeV}-1.3<\eta<-0.8$ data set. Fit parameters can be found in table 12 .

Open Access. This article is distributed under the terms of the Creative Commons Attribution License (CC-BY 4.0), which permits any use, distribution and reproduction in any medium, provided the original author(s) and source are credited.

\section{References}

[1] P. Di Vecchia, The birth of string theory, Lect. Notes Phys. 737 (2008) 59 [arXiv: 0704.0101] [INSPIRE].

[2] G. 't Hooft, A planar diagram theory for strong interactions, Nucl. Phys. B 72 (1974) 461 [INSPIRE].

[3] J.M. Maldacena, The large- $N$ limit of superconformal field theories and supergravity, Int. J. Theor. Phys. 38 (1999) 1113 [hep-th/9711200] [INSPIRE]. 
[4] J.M. Maldacena, Wilson loops in large-N field theories, Phys. Rev. Lett. 80 (1998) 4859 [hep-th/9803002] [INSPIRE].

[5] E. Witten, Anti-de Sitter space and holography, Adv. Theor. Math. Phys. 2 (1998) 253 [hep-th/9802150] [INSPIRE].

[6] S.S. Gubser, I.R. Klebanov and A.M. Polyakov, Gauge theory correlators from noncritical string theory, Phys. Lett. B 428 (1998) 105 [hep-th/9802109] [INSPIRE].

[7] O. Aharony, S.S. Gubser, J.M. Maldacena, H. Ooguri and Y. Oz, Large-N field theories, string theory and gravity, Phys. Rept. 323 (2000) 183 [hep-th/9905111] [INSPIRE].

[8] S.S. Gubser, I.R. Klebanov and A.M. Polyakov, A semiclassical limit of the gauge/string correspondence, Nucl. Phys. B 636 (2002) 99 [hep-th/0204051] [INSPIRE].

[9] H.D. Politzer, Reliable perturbative results for strong interactions?, Phys. Rev. Lett. 30 (1973) 1346 [INSPIRE].

[10] D.J. Gross and F. Wilczek, Ultraviolet behavior of non-Abelian gauge theories, Phys. Rev. Lett. 30 (1973) 1343 [INSPIRE].

[11] V.M. Braun, G.P. Korchemsky and D. Mueller, The uses of conformal symmetry in QCD, Prog. Part. Nucl. Phys. 51 (2003) 311 [hep-ph/0306057] [InSPIRE].

[12] Z. Bern, L.J. Dixon and D.A. Kosower, $N=4$ super-Yang-Mills theory, $Q C D$ and collider physics, Comptes Rendus Physique 5 (2004) 955 [hep-th/0410021] [INSPIRE].

[13] J. Polchinski and M.J. Strassler, Hard scattering and gauge/string duality, Phys. Rev. Lett. 88 (2002) 031601 [hep-th/0109174] [INSPIRE].

[14] R.C. Brower and C.-I. Tan, Hard scattering in the M-theory dual for the QCD string, Nucl. Phys. B 662 (2003) 393 [hep-th/0207144] [INSPIRE].

[15] R.C. Brower, J. Polchinski, M.J. Strassler and C.-I. Tan, The pomeron and gauge/string duality, JHEP 12 (2007) 005 [hep-th/0603115] [INSPIRE].

[16] R.C. Brower, M.J. Strassler and C.-I. Tan, On the eikonal approximation in AdS space, JHEP 03 (2009) 050 [arXiv: 0707.2408] [INSPIRE].

[17] R.C. Brower, M.J. Strassler and C.-I. Tan, On the pomeron at large 't Hooft coupling, JHEP 03 (2009) 092 [arXiv:0710.4378] [INSPIRE].

[18] L. Cornalba, M.S. Costa, J. Penedones and R. Schiappa, Eikonal approximation in AdS/CFT: conformal partial waves and finite $N$ four-point functions, Nucl. Phys. B 767 (2007) 327 [hep-th/0611123] [INSPIRE].

[19] L. Cornalba, M.S. Costa, J. Penedones and R. Schiappa, Eikonal approximation in AdS/CFT: from shock waves to four-point functions, JHEP 08 (2007) 019 [hep-th/0611122] [INSPIRE].

[20] L. Cornalba, Eikonal methods in AdS/CFT: Regge theory and multi-reggeon exchange, arXiv: 0710.5480 [INSPIRE].

[21] L. Cornalba, M.S. Costa and J. Penedones, Eikonal methods in AdS/CFT: BFKL pomeron at weak coupling, JHEP 06 (2008) 048 [arXiv:0801.3002] [INSPIRE].

[22] L. Cornalba, M.S. Costa and J. Penedones, Deep inelastic scattering in conformal QCD, JHEP 03 (2010) 133 [arXiv:0911.0043] [INSPIRE].

[23] M.S. Costa, V. Goncalves and J. Penedones, Conformal Regge theory, JHEP 12 (2012) 091 [arXiv: 1209.4355] [INSPIRE]. 
[24] N. Anderson, S.K. Domokos, J.A. Harvey and N. Mann, Central production of $\eta$ and $\eta^{\prime}$ via double pomeron exchange in the Sakai-Sugimoto model, Phys. Rev. D 90 (2014) 086010 [arXiv:1406.7010] [INSPIRE].

[25] N. Anderson, S. Domokos and N. Mann, Central production of $\eta$ via double pomeron exchange and double reggeon exchange in the Sakai-Sugimoto model, Phys. Rev. D 96 (2017) 046002 [arXiv: 1612.07457] [INSPIRE].

[26] J. Polchinski and M.J. Strassler, Deep inelastic scattering and gauge/string duality, JHEP 05 (2003) 012 [hep-th/0209211] [INSPIRE].

[27] R.C. Brower, M. Djuric, I. Sarcevic and C.-I. Tan, String-gauge dual description of deep inelastic scattering at small-x, JHEP 11 (2010) 051 [arXiv:1007.2259] [INSPIRE].

[28] M.S. Costa and M. Djuric, Deeply virtual Compton scattering from gauge/gravity duality, Phys. Rev. D 86 (2012) 016009 [arXiv: 1201.1307] [INSPIRE].

[29] M.S. Costa, M. Djurić and N. Evans, Vector meson production at low x from gauge/gravity duality, JHEP 09 (2013) 084 [arXiv: 1307.0009] [inSPIRE].

[30] R.C. Brower, M. Djurić, T. Raben and C.-I. Tan, Holographic approach to deep inelastic scattering at small-x at high energy, PoS (DIS2015) 089 [arXiv: 1508.05063] [INSPIRE].

[31] R. Nishio and T. Watari, High-energy photon-hadron scattering in holographic QCD, Phys. Rev. D 84 (2011) 075025 [arXiv:1105.2999] [INSPIRE].

[32] E. Koile, N. Kovensky and M. Schvellinger, Deep inelastic scattering cross sections from the gauge/string duality, JHEP 12 (2015) 009 [arXiv: 1507.07942] [INSPIRE].

[33] A. Ballon-Bayona, R. Carcassés Quevedo, M.S. Costa and M. Djurić, Soft pomeron in holographic QCD, Phys. Rev. D 93 (2016) 035005 [arXiv:1508.00008] [inSPIRE].

[34] M. Hentschinski, A. Sabio Vera and C. Salas, Hard to soft pomeron transition in small-x deep inelastic scattering data using optimal renormalization, Phys. Rev. Lett. 110 (2013) 041601 [arXiv: 1209.1353] [INSPIRE].

[35] L. Frankfurt, M. Strikman and C. Weiss, Small-x physics: from HERA to LHC and beyond, Ann. Rev. Nucl. Part. Sci. 55 (2005) 403 [hep-ph/0507286] [inSPIRE].

[36] C. Marquet and L. Schoeffel, Geometric scaling in diffractive deep inelastic scattering, Phys. Lett. B 639 (2006) 471 [hep-ph/0606079] [INSPIRE].

[37] C. Marquet, A unified description of diffractive deep inelastic scattering with saturation, Phys. Rev. D 76 (2007) 094017 [arXiv:0706.2682] [INSPIRE].

[38] E. Avsar and G. Gustafson, Geometric scaling and QCD dynamics in DIS, JHEP 04 (2007) 067 [hep-ph/0702087] [INSPIRE].

[39] M.M. Block, L. Durand and P. Ha, Connection of the virtual $\gamma^{*} p$ cross section of ep deep inelastic scattering to real $\gamma p$ scattering and the implications for $\nu N$ and ep total cross sections, Phys. Rev. D 89 (2014) 094027 [arXiv: 1404.4530] [InSPIRE].

[40] R.P. Feynman, Very high-energy collisions of hadrons, Phys. Rev. Lett. 23 (1969) 1415 [INSPIRE].

[41] J. Benecke, T.T. Chou, C.-N. Yang and E. Yen, Hypothesis of limiting fragmentation in high-energy collisions, Phys. Rev. 188 (1969) 2159 [INSPIRE].

[42] K.G. Wilson, Some experiments on multiple production, Cornell preprint CLNS-131-1970, Cornell University, Ithaca NY U.S.A., [INSPIRE]. 
[43] M.J. Strassler, Why unparticle models with mass gaps are examples of hidden valleys, arXiv:0801.0629 [INSPIRE].

[44] D.M. Hofman and J. Maldacena, Conformal collider physics: energy and charge correlations, JHEP 05 (2008) 012 [arXiv:0803.1467] [INSPIRE].

[45] A.V. Belitsky, S. Hohenegger, G.P. Korchemsky, E. Sokatchev and A. Zhiboedov, From correlation functions to event shapes, Nucl. Phys. B 884 (2014) 305 [arXiv:1309.0769] [INSPIRE].

[46] A.V. Belitsky, S. Hohenegger, G.P. Korchemsky, E. Sokatchev and A. Zhiboedov, Event shapes in $N=4$ super-Yang-Mills theory, Nucl. Phys. B 884 (2014) 206 [arXiv:1309.1424] [INSPIRE].

[47] A.V. Belitsky, S. Hohenegger, G.P. Korchemsky, E. Sokatchev and A. Zhiboedov, Energy-energy correlations in $N=4$ supersymmetric Yang-Mills theory, Phys. Rev. Lett. 112 (2014) 071601 [arXiv:1311.6800] [INSPIRE].

[48] G.F. Sterman and S. Weinberg, Jets from quantum chromodynamics, Phys. Rev. Lett. 39 (1977) 1436 [INSPIRE].

[49] L.S. Brown and S.D. Ellis, Energy-energy correlations in electron-positron annihilations, Phys. Rev. D 24 (1981) 2383 [InSPIRE].

[50] J. Maldacena, D. Simmons-Duffin and A. Zhiboedov, Looking for a bulk point, JHEP 01 (2017) 013 [arXiv: 1509.03612] [INSPIRE].

[51] R.C. Brower, M.S. Costa, M. Djurić, T. Raben and C.-I. Tan, Strong coupling expansion for the conformal pomeron/odderon trajectories, JHEP 02 (2015) 104 [arXiv:1409.2730] [INSPIRE].

[52] C.P. Herzog, S. Paik, M.J. Strassler and E.G. Thompson, Holographic double diffractive scattering, JHEP 08 (2008) 010 [arXiv:0806.0181] [INSPIRE].

[53] E.A. Kuraev, L.N. Lipatov and V.S. Fadin, The Pomeranchuk singularity in non-Abelian gauge theories, Sov. Phys. JETP 45 (1977) 199 [Zh. Eksp. Teor. Fiz. 72 (1977) 377] [INSPIRE].

[54] I.I. Balitsky and L.N. Lipatov, The Pomeranchuk singularity in quantum chromodynamics, Sov. J. Nucl. Phys. 28 (1978) 822 [Yad. Fiz. 28 (1978) 1597] [InSPIRE].

[55] A.V. Kotikov, L.N. Lipatov, A.I. Onishchenko and V.N. Velizhanin, Three loop universal anomalous dimension of the Wilson operators in $N=4$ SUSY Yang-Mills model, Phys. Lett. B 595 (2004) 521 [Erratum ibid. B 632 (2006) 754] [hep-th/0404092] [InSPIRE].

[56] C.E. DeTar, J.H. Weis, K. Kang and C.-I. Tan, Duality and single-particle production, Phys. Rev. D 4 (1971) 425 [InSPIRE].

[57] M.A. Virasoro, Simple dual-resonance model for inclusive reactions, Phys. Rev. D 3 (1971) 2834 [INSPIRE].

[58] D. Gordon and G. Veneziano, Inclusive reactions and dual-resonance models, Phys. Rev. D 3 (1971) 2116 [INSPIRE].

[59] L. Clavelli, Particle production in the double dual Pomeron region, Nucl. Phys. B 154 (1979) 47 [INSPIRE].

[60] J.P. Ader and L. Clavelli, A Regge cut model for high $p_{T}$ behavior, Phys. Lett. B 66 (1977) 97 [INSPIRE]. 
[61] O. Andreev and W. Siegel, Quantized tension: stringy amplitudes with Regge poles and parton behavior, Phys. Rev. D 71 (2005) 086001 [hep-th/0410131] [INSPIRE].

[62] O. Andreev, A few comments on the high-energy behavior of string scattering amplitudes in warped space-times, Phys. Rev. D 70 (2004) 027901 [hep-th/0402017] [INSPIRE].

[63] C. Csáki, H. Ooguri, Y. Oz and J. Terning, Glueball mass spectrum from supergravity, JHEP 01 (1999) 017 [hep-th/9806021] [INSPIRE].

[64] H. Ooguri, H. Robins and J. Tannenhauser, Glueballs and their Kaluza-Klein cousins, Phys. Lett. B 437 (1998) 77 [hep-th/9806171] [INSPIRE].

[65] R. de Mello Koch, A. Jevicki, M. Mihailescu and J.P. Nunes, Evaluation of glueball masses from supergravity, Phys. Rev. D 58 (1998) 105009 [hep-th/9806125] [INSPIRE].

[66] F. Brünner, D. Parganlija and A. Rebhan, Glueball decay rates in the Witten-Sakai-Sugimoto model, Phys. Rev. D 91 (2015) 106002 [Erratum ibid. D 93 (2016) 109903] [arXiv: 1501.07906] [INSPIRE].

[67] K. Hashimoto, C.-I. Tan and S. Terashima, Glueball decay in holographic QCD, Phys. Rev. D 77 (2008) 086001 [arXiv:0709.2208] [INSPIRE].

[68] F. Brünner, D. Parganlija and A. Rebhan, Holographic glueball decay, Acta Phys. Polon. Supp. 7 (2014) 533 [arXiv: 1407.6914] [INSPIRE].

[69] F. Brünner, D. Parganlija and A. Rebhan, Top-down holographic glueball decay rates, AIP Conf. Proc. 1701 (2016) 090007 [arXiv:1502. 00456] [INSPIRE].

[70] J. Erdmenger, N. Evans, I. Kirsch and E. Threlfall, Mesons in gauge/gravity duals - a review, Eur. Phys. J. A 35 (2008) 81 [arXiv:0711.4467] [InSPIRE].

[71] L.N. Lipatov, Small $x$ physics in perturbative QCD, Phys. Rept. 286 (1997) 131 [hep-ph/9610276] [INSPIRE].

[72] L.S. Kisslinger and W.-H. Ma, Pomeron and the Reggeized glueball/sigma, Phys. Lett. B 485 (2000) 367 [hep-ph/9905479] [INSPIRE].

[73] S.J. Brodsky and G.R. Farrar, Scaling laws at large transverse momentum, Phys. Rev. Lett. 31 (1973) 1153 [INSPIRE].

[74] S.J. Brodsky and G.R. Farrar, Scaling laws for large momentum transfer processes, Phys. Rev. D 11 (1975) 1309 [INSPIRE].

[75] V.A. Matveev, R.M. Muradian and A.N. Tavkhelidze, Automodellism in the large-angle elastic scattering and structure of hadrons, Lett. Nuovo Cim. 7 (1973) 719 [INSPIRE].

[76] A.H. Mueller, $O(2,1)$ analysis of single particle spectra at high-energy, Phys. Rev. D 2 (1970) 2963 [INSPIRE].

[77] R.C. Brower, M. Djuric and C.-I. Tan, Diffractive Higgs production by AdS pomeron fusion, JHEP 09 (2012) 097 [arXiv: 1202.4953] [INSPIRE].

[78] R. Brower, M. Djuric and C.-I. Tan, Holographic double diffraction of Higgs and the AdS graviton/pomeron holographic double diffraction of Higgs and the AdS/CFT graviton/pomeron, Int. J. Mod. Phys. A 29 (2014) 1446013 [arXiv:1412.3060] [InSPIRE].

[79] O. Andreev and V.I. Zakharov, Heavy-quark potentials and AdS/QCD, Phys. Rev. D 74 (2006) 025023 [hep-ph/0604204] [INSPIRE].

[80] J.I. Kapusta and T. Springer, Potentials for soft wall AdS/QCD, Phys. Rev. D 81 (2010) 086009 [arXiv: 1001.4799] [INSPIRE]. 
[81] O. Aharony, M. Berkooz, D. Tong and S. Yankielowicz, Confinement in anti-de Sitter space, JHEP 02 (2013) 076 [arXiv:1210.5195] [INSPIRE].

[82] E. Witten, Anti-de Sitter space, thermal phase transition and confinement in gauge theories, Adv. Theor. Math. Phys. 2 (1998) 505 [hep-th/9803131] [InSPIRE].

[83] U. Gürsoy, E. Kiritsis, L. Mazzanti and F. Nitti, Holography and thermodynamics of $5 D$ dilaton-gravity, JHEP 05 (2009) 033 [arXiv: 0812.0792] [INSPIRE].

[84] A. Karch, E. Katz, D.T. Son and M.A. Stephanov, Linear confinement and AdS/QCD, Phys. Rev. D 74 (2006) 015005 [hep-ph/0602229] [INSPIRE].

[85] G.F. de Teramond and S.J. Brodsky, Hadronic spectrum of a holographic dual of QCD, Phys. Rev. Lett. 94 (2005) 201601 [hep-th/0501022] [INSPIRE].

[86] U. Gürsoy and E. Kiritsis, Exploring improved holographic theories for QCD: part I, JHEP 02 (2008) 032 [arXiv:0707.1324] [INSPIRE].

[87] U. Gürsoy, E. Kiritsis and F. Nitti, Exploring improved holographic theories for QCD: part II, JHEP 02 (2008) 019 [arXiv:0707.1349] [INSPIRE].

[88] B. Batell and T. Gherghetta, Dynamical soft-wall AdS/QCD, Phys. Rev. D 78 (2008) 026002 [arXiv: 0801.4383] [INSPIRE].

[89] R.C. Brower, M. Djuric and C.-I. Tan, Diffractive Higgs production by AdS pomeron fusion, JHEP 09 (2012) 097 [arXiv: 1202.4953] [InSPIRE].

[90] R.C. Brower, M.S. Costa, M. Djuric and C.-I. Tan, Deeply virtual Compton scattering and Higgs production using the pomeron in AdS, in Rencontres de Physique des Particules, Montpellier France, 14-16 May 2012 [arXiv: 1210.1511] [INSPIRE].

[91] R. Brower, R.C. Brower, M. Djurić, T. Raben and C.-I. Tan, Towards holographic QCD: AdS/CFT, confinement deformation and DIS at small-x, in Proceedings, $49^{\text {th }}$ Rencontres de Moriond on QCD and High Energy Interactions, La Thuile Italy, 22-29 March 2014, pg. 173 [arXiv: 1412.3443] [INSPIRE].

[92] J.H. Weis, Factorization of discontinuities of multi-Regge amplitudes, Nucl. Phys. B 71 (1974) 342 [INSPIRE].

[93] ATLAS collaboration, Charged-particle distributions in $\sqrt{s}=13 \mathrm{TeV}$ pp interactions measured with the ATLAS detector at the LHC, Phys. Lett. B 758 (2016) 67 [arXiv: 1602.01633] [INSPIRE].

[94] ATLAS collaboration, Charged-particle distributions in pp interactions at $\sqrt{s}=8 \mathrm{TeV}$ measured with the ATLAS detector, Eur. Phys. J. C 76 (2016) 403 [arXiv:1603.02439] [INSPIRE].

[95] ALICE collaboration, Transverse momentum dependence of inclusive primary charged-particle production in $p$-Pb collisions at $\sqrt{s_{\mathrm{NN}}}=5.02 \mathrm{TeV}$, Eur. Phys. J. C 74 (2014) 3054 [arXiv: 1405.2737] [INSPIRE].

[96] E. Levin and A.H. Rezaeian, Gluon saturation and inclusive hadron production at LHC, Phys. Rev. D 82 (2010) 014022 [arXiv: 1005.0631] [INSPIRE].

[97] ATLAS collaboration, The ATLAS experiment at the CERN Large Hadron Collider, 2008 JINST 3 S08003 [INSPIRE].

[98] M.J. Tannenbaum, Hard and soft physics at RHIC with implications for LHC, PoS (High-pT physics09) 004 [arXiv:0904.4363] [INSPIRE].

[99] R. Blankenbecler, S.J. Brodsky and J.F. Gunion, Inclusive processes at high transverse momentum, Phys. Lett. B 42 (1972) 461 [INSPIRE]. 
[100] T. Sakai and S. Sugimoto, Low energy hadron physics in holographic QCD, Prog. Theor. Phys. 113 (2005) 843 [hep-th/0412141] [INSPIRE].

[101] D. Kharzeev, E. Levin and K. Tuchin, Multi-particle production and thermalization in high-energy QCD, Phys. Rev. C 75 (2007) 044903 [hep-ph/0602063] [INSPIRE].

[102] A.A. Bylinkin and M.G. Ryskin, Secondary hadron distributions in two component model, Phys. Rev. D 90 (2014) 017501 [arXiv:1404.4739] [INSPIRE].

[103] D. Kharzeev, Hawking-Unruh phenomenon in the parton language, Eur. Phys. J. A 29 (2006) 83 [INSPIRE].

[104] P. Castorina, D. Kharzeev and H. Satz, Thermal hadronization and Hawking-Unruh radiation in QCD, Eur. Phys. J. C 52 (2007) 187 [arXiv:0704.1426] [INSPIRE].

[105] A. Bylinkin, N.S. Chernyavskaya and A.A. Rostovtsev, Predictions on the transverse momentum spectra for charged particle production at LHC-energies from a two component model, Eur. Phys. J. C 75 (2015) 166 [arXiv:1501.05235] [INSPIRE].

[106] S.J. Brodsky, H.J. Pirner and J. Raufeisen, Scaling properties of high $p_{T}$ inclusive hadron production, Phys. Lett. B 637 (2006) 58 [hep-ph/0510315] [INSPIRE].

[107] G. Mack, D-independent representation of conformal field theories in $D$ dimensions via transformation to auxiliary dual resonance models. Scalar amplitudes, arXiv:0907.2407 [INSPIRE].

[108] C.-L. Jen, K. Kang, P. Shen and C.-I. Tan, Dual-amplitude analysis of two-particle productions, Phys. Rev. Lett. 27 (1971) 458 [INSPIRE].

[109] B. Hasslacher, C.S. Hsue and D.K. Sinclair, Dual-resonance-model implications for two-particle spectra in inclusive reactions, Phys. Rev. D 4 (1971) 3089 [INSPIRE].

[110] G. Chachamis, M. Hentschinski, A. Sabio Vera and C. Salas, Exclusive central production of heavy quarks at the LHC, arXiv:0911.2662 [INSPIRE].

[111] D. Ebert, R.N. Faustov and V.O. Galkin, Spectroscopy and Regge trajectories of heavy quarkonia in the relativistic quark model, in Proceedings, $17^{\text {th }}$ International Seminar on High Energy Physics (Quarks 2012), Yaroslavl Russia, 4-7 June 2012 [INSPIRE].

[112] S.J. Brodsky, G.F. de Téramond, H.G. Dosch and C. Lorcé, Universal effective hadron dynamics from superconformal algebra, Phys. Lett. B 759 (2016) 171 [arXiv:1604.06746] [INSPIRE].

[113] S.J. Brodsky and R.F. Lebed, QCD dynamics of tetraquark production, Phys. Rev. D 91 (2015) 114025 [arXiv: 1505.00803] [INSPIRE].

[114] J. Sonnenschein and D. Weissman, A tetraquark or not a tetraquark? A holography inspired stringy hadron (HISH) perspective, Nucl. Phys. B 920 (2017) 319 [arXiv:1606.02732] [INSPIRE].

[115] H.P. Stapp, Inclusive cross-sections are discontinuities, Phys. Rev. D 3 (1971) 3177 [INSPIRE].

[116] C.-I. Tan, Elementary discontinuity formulas and Mueller's Regge hypothesis, Phys. Rev. D 4 (1971) 2412 [INSPIRE].

[117] Z. Komargodski, M. Kulaxizi, A. Parnachev and A. Zhiboedov, Conformal field theories and deep inelastic scattering, Phys. Rev. D 95 (2017) 065011 [arXiv:1601.05453] [InSPIRE].

[118] D.J. Gross and P.F. Mende, The high-energy behavior of string scattering amplitudes, Phys. Lett. B 197 (1987) 129 [INSPIRE]. 